\title{
SUBESPAÇOS \\ COMPLEMENTADOS \\ NA SOMA DE \\ ESPAÇOS DE BANACH
}

Odirlei Silva Jesus

\author{
DISSERTAÇÃO APRESENTADA \\ $\mathrm{AO}$ \\ INSTITUTO DE MATEMÁTICA E ESTATÍSTICA
}

DA

UNIVERSIDADE DE SÃO PAULO

PARA

OBTENÇÃO DO GRAU DE MESTRE

EM

MATEMÁTICA

Área de concentração: Análise

Orientador: Prof. Dr. Elói Medina Galego

Durante a elaboração deste trabalho o autor recebeu auxilio financeiro da FAPESP.

São Paulo, 13 de setembro de 2002 


\section{Subespaços Complementados na Soma de Espaços de Banach}

Este exemplar corresponde à redação final da dissertação devidamente corrigida e defendida por Odirlei Silva Jesus e aprovada pela comissão julgadora.

São Paulo, 13 de setembro de 2002.

Banca examinadora:

- Prof. Dr. Elói Medina Galego (orientador) - IME-USP

- Prof. Dr. Chaim Samuel Hönig - IME-USP

- Prof. Dr. Antonio Roberto da Silva - UFRJ 
"Nenhum sucesso na vida compensa o fracasso no lar."

David O. Mckay (1873-1970)

À minha esposa Luciana, pelo apoio e confiança durante todos estes anos. 


\section{Resumo}

O objetivo desse trabalho é apresentar soluções parciais recentes do seguinte problema em aberto:

Problema. Sejam $X$ e $Y$ espaços de Banach. É verdade que todo subespaço complementado na soma direta de $X$ com $Y$ é isomorfo a soma direta de um subespaço complementado de $X$ com um subespaço complementado de $Y$ ?

\section{Abstract}

The purpose of this work is to present recent partial solutions of the following open problem:

Problem. Let $X$ and $Y$ be Banach spaces. Is it true that every complemented subspace in the direct sum of $X$ and $Y$ is isomorphic to the direct sum of a complemented subspace of $X$ and a complemented subspace of $Y$ ? 


\section{Agradecimentos}

- Ao Prof. Elói Medina Galego por sua paciente orientação, incentivo e firmeza de ânimo em todos os momentos do trabalho.

- A minha esposa Luciana por seu constante incentivo, apoio, paciência e amor.

- Aos meus pais.

- Ao Prof. Manuel González Ortiz pelas dúvidas esclarecidas e por sua obra literária na área.

- Ao colega Antonio Ronaldo Garcia por sua amizade, incentivo e também pelo apoio com o editor de texto.

- A FAPESP pelo suporte financeiro.

- Ao Senhor, por ter preparado o caminho para que este trabalho fosse concretizado. 


\section{Índice}

Notações e definições básicas

Introdução

1 Preliminares 7

2 Operadores de Fredholm $\quad 17$

2.1 Módulo mínimo de um operador . . . . . . . . . . . . . . . . 17

2.2 Operadores de Fredholm . . . . . . . . . . . . . . . . . . 23

2.3 Ideais de operadores lineares . . . . . . . . . . . . . . . . 27

2.4 Teoria de perturbação de operadores de Fredholm . . . . . . . . . . . . . 33

2.5 Caracterizações de operadores de Fredholm . . . . . . . . . . . . . . . . 43

3 Teoria espectral e funções de operadores lineares 49

4 Subespaços complementados na soma de espaços de Banach $\quad 59$

5 Operadores inessenciais e improjetivos $\quad 75$

5.1 Operadores inessenciais . . . . . . . . . . . . . . 76 
5.2 Operadores improjetivos . . . . . . . . . . . . . 85

5.3 Operadores inessenciais e improjetivos . . . . . . . . . . . . . . 89

5.4 Exemplo de um operador improjetivo que não é inessencial . . . . . . . . . 97

6 Subespaços complementados na soma de espaços de Banach essencialmente incomparáveis 


\section{Notações e definições básicas}

Denotaremos por $\mathbb{C}$ o conjunto dos números complexos, por $\mathbb{R}$ o conjunto dos números reais, por $\mathbb{Z}$ o conjunto dos números inteiros e por $\mathbb{N}$ o conjunto dos números naturais.

Dado um espaço vetorial $X$ e um subconjunto $M$ de $X$, indicaremos por $[M]$ o subespaço gerado por $M$ em $X$. Se $x_{1}, \ldots, x_{n} \in X$ denotaremos por $\left[x_{1}, \ldots, x_{n}\right]$ o subespaço de $X$ gerado pelos vetores $x_{1}, \ldots, x_{n}$.

Dado um subconjunto $A$ de um espaço vetorial $X$ e pontos $c, d \in A$, denotaremos por $[c, d]$ o conjunto $\{\lambda c+(1-\lambda) d: 0 \leq \lambda \leq 1\}$, ou seja, o segmento de reta com extremidades $c$ e $d$. Denotaremos por $X-A$ o subconjunto de $X$ de todos os elementos que não pertencem a $A$.

Seja $X$ um espaço vetorial normado. A métrica $d$ induzida pela norma é definida por $d(x, y)=\|x-y\|, \forall x, y \in X$. Para $x \in X$ e um subconjunto $M$ de $X$, denotaremos por $d(x, M)$ a distância de $X$ a $M$, isto é, $d(x, M)=\inf \{\|x-m\|: m \in M\}$.

Seja $X$ um espaço de Banach. Indicaremos por $B_{X}$ a bola unitária fechada de centro no elemento nulo de $X$ e raio um, isto é, $B_{X}=\{x \in X:\|x\| \leq 1\}$. Dado $x \in X$ e $\varepsilon>0$ denotaremos por $B(x, \varepsilon)$ a bola aberta de centro $x$ e raio $\varepsilon$, isto é, $B(x, \varepsilon)=\{y \in X$ : $\|x-y\|<\varepsilon\}$. Da.da uma seqüência $\left\{x_{n}\right\}$ em $X$ e dado $x \in X$, usaremos a notação $x_{n} \rightarrow x$ para denotar que a seqüência $\left\{x_{n}\right\}$ converge a $x$ quando $n \rightarrow \infty$.

Se $X$ é um espaço topológico e $A$ é um subconjunto de $X$, então $\bar{A}$ indica o fecho de $A$ em $X$. Diremos que $A$ é denso em $X$ se $\vec{A}=X$.

Sejam $X$ e $Y$ espaços normados. $L(X, Y)$ denotará o espaço de Banach dos operadores 
lineares contínuos de $X$ em $Y$ com a norma $\|T\|=\sup \{T(x):\|x\|=1\}, \forall T \in L(X, Y)$. Se $X=Y$ então denotaremos $L(X, X)$ por $L(X)$.

Nesse trabalho será necessário estudarmos operadores lineares $T$ definidos em um subespaço do espaço vetorial $X$ a valores em um espaço vetorial $Y$, e então denotaremos por $D(T)$ o domínio do operador $T$, por $R(T)$ a imagem de $T$, ou seja, $R(T)=\{T x: x \in X\}$ e por $N(T)$ o núcleo $\{x \in X: T(x)=0\}$ de $T$. Se $M$ é um subespaço de $X$ então denotaremos por $T_{\left.\right|_{M}}$ a restrição do operador $T$ a $M$.

Seja $X$ um espaço de Banach. Denotaremos por $I_{X}$ o operador identidade $I_{X}: X \rightarrow X$ definido por $I_{X}(x)=x, \forall x \in X$.

Se $X$ é um espaço de Banach, $M \subset X$ é um subespaço de $X$ e $T \in L(X)$ então diremos que $M$ é invariante por $T$ se $T(M) \subset M$.

Finalmente $L$ denotará a classe de todos os operadores lineares contínuos entre quaisquer espaços normados.

Sejam $X$ e $Y$ espaços de Banach. $T \in L(X, Y)$ é um isomorfismo de $X$ sobre $Y$ se $T$ é injetor e a imagem de $T$ é $Y$. Dizemos que $X$ é isomorfo a $Y$ se existe um isomorfismo $T$ de $X$ sobre $Y$. Se, além disso, $\|T(x)\|=\|x\|, \forall x \in X$, então $T$ é dito uma isometria, e diremos que $X$ e $Y$ são isométricos.

Seja $X$ um espaço de Banach e $M$ um subespaço de $X$. Chamaremos de inclusão canônica de $M$ em $X$ a função $J_{M}^{X}: M \rightarrow X$ definida por $J_{M}^{X}(m)=m, \forall m \in M$.

Se $X$ é um espaço de Banach denotaremos por $\operatorname{dim}(X)$ a dimensão de $X$, que pode ser finita ou infinita. Seja $M$ um subespaço fechado de $X$. Denotaremos por $X / M$ o espaço de Banach quociente $\{[x]: x \in X\}$, onde $[x]$ denota a classe de equivalência $x+M$ de $x$ com a norma $\|[x]\|=d(x, M)$. Denotaremos por $\operatorname{codim}(M)$ a codimensão de $M$ em $X$, isto é, a dimensão do espaço quociente $X / M$. Indicaremos que $M$ tem codimensão finita em $X$ pela notação $M \in \operatorname{Cod}(X)$. Por $Q_{M}$ denotaremos a aplicação quociente canônica. de $X$ em $X / M$ definida por $Q_{M}(x)=[x], \forall x \in X$.

Dados dois espaços de Banach $X$ e $Y$ denotaremos a soma direta externa de $X$ com $Y$ por $X \oplus Y=\{(x, y): x \in X, y \in Y\}$ com a norma do máximo $\|(x, y)\|=\max (\|x\|,\|y\|)$.

Por $c_{o}(\mathbb{N})$ denotaremos o espaço de Banach das seqüências convergentes a zero com a. norma do supremo. 
Seja $p \geq 1$ em $\mathbb{R}$. Por $l_{p}(\mathbb{N})$ denotaremos o espaço de Banach das seqüências p-somáveis com a norma $\left\|\left\{a_{n}\right\}_{n}\right\|_{l_{p}}=\left(\sum_{n=1}^{\infty}\left|a_{n}\right|^{p}\right)^{\frac{1}{p}}$.

Por $C[0,1]$ denotaremos o espaço de Banach das funções $f:[0,1] \rightarrow \mathbb{R}$ contínuas em $[0,1]$ com a norma $\|f\|=\sup \{|f(x)|: x \in[0,1]\}$.

O símbolo denota o fim de uma demonstração. 


\section{Introdução}

O objetivo desse trabalho é apresentar conceitos básicos que motivam a pesquisa da solução do seguinte problema em aberto:

Problema 1. Sejam $X$ e $Y$ espaços de Banach. É verdade que todo subespaço complementado na soma direta de $X$ com $Y$ é isomorfo a soma direta de um subespaço complementado de $X$ com um subespaço complementado de $Y$ ?

Nessa dissertação utilizaremos resultados da teoria espectral de operadores lineares e da teoria de pertubação de operadores de Fredholm por operadores estritamente singulares (veja Definição 41) e, mais geralmente, por operadores pertencentes a um $\Phi$-ideal (veja Definição 98), para apresentar soluções parciais para o Problema 1 no caso em que todo operador linear contínuo de $X$ em $Y$ é estritamente singular (veja Teorema 149) e também no caso em que todo operador linear contínuo de $X$ em $Y$ é inessencial (veja Teorema 203).

Destacamos que o seguinte caso particular do Problema 1 também está em aberto:

Problema 2. Sejam $X$ e $Y$ espaços de Banach tais que todo operador linear contínuo de $X$ em $Y$ é improjetivo (veja Definição 171). É verdade que todo subespaço complementado na soma direta de $X$ com $Y$ é isomorfo a soma direta de um subespaço complementado de $X$ com um subespaço complementado de $Y$ ?

No decorrer de nosso trabalho apresentaremos alguns resultados sobre operadores inessenciais e operadores improjetivos [1], [2], [3]. Mostraremos que todo operador inessencial é um operador improjetivo [3] e exibiremos alguns casos particulares de espaços de Banach para os quais as classes dos operadores improjetivos e dos operadores inessenciais 
coincidem. Daremos exemplos de espaços de Banach para os quais existe um operador que é improjetivo mas não é-inessencial [2]. Na descrição desses contra-exemplos utilizaremos um espaço de Banach indecomponível construído por Gowers, W.T. e Maurey, B. [11], e uma caracterização dos espaços de Banach indecomponíveis em termos de operadores improjetivos [2]. Tais exemplos conseguidos por Aiena, P. e González, M. no ano 2000 respondem negativamente a uma conjectura proposta por González, M. em [10].

Mais concretamente, no Capítulo 1 apresentaremos algumas definições e resultados preliminares que utilizaremos no decorrer desse trabalho.

No capítulo 2 veremos o conceito de operadores de Fredholm e os principais resultados sobre esses operadores, onde destacaremos os resultados de pertubação de operadores de Fredholm por operadores estritamente singulares e por operadores em um $\Phi$-ideal.

No Capítulo 3 serão apresentados resultados de teoria espectral de operadores lineares que são utilizados nas demonstrações dos teoremas apresentados nos Capítulos 4 e 6 sobre subespaços complementados na soma direta de espaços de Banach.

No Capítulo 4 veremos que o Problema 1 tem solução positiva no caso em que todo operador linear contínuo entre os espaços de Banach considerados é estritamente singular. Esse resultado foi obtido por Wo.jtaszczyk, P. [33] em 1976.

No Capítulo 5 introduziremos os conceitos de operadores inessenciais e improjetivos e estudaremos alguns resultados destas classes de operadores em preparação para mostrar que o Problema 1 tem solução positiva no caso em que todo operador linear contínuo entre os espaços de Banach em questão é inessencial [10]. Esse resultado será apresentado no Capítulo 6 e foi obtido por González, M. em 1994. 


\section{Preliminares}

Nessa seção apresentaremos algumas definições e resultados sobre a teoria dos espaços de Banach que serão utilizados nos próximos capítulos.

Teorema 1 (Teorema de Liouville). ([14], Proposição 46.6) Se $f: \mathbb{C} \rightarrow \mathbb{C}$ é uma função analítica e limitada em todo o dominio $\mathbb{C}$ então $f$ é constante.

Proposição 2. ([17], (11.4)) Todo conjunto $X \subset \mathbb{R}^{n}$ contém um subconjunto E que é enumerável e denso em $X$.

Proposição 3. ([8], Teorema 1.4.12) A soma de dois subespaços fechados de um espaço vetorial normado é fechada quando um dos subespaços é de dimensão finita.

Definição 4. Sejam $X$ um espaço de Banach e $P \in L(X)$. Dizemos que $P$ é uma projeção em $X$ se $P(P(x))=P(x), \forall x \in X$, ou seja, $P^{2}=P$.

Definição 5. Seja $X$ um espaço de Banach e $M$ um subespaço de $X$. Diremos que $M$ é complementado em $X$ se existe uma projeção $P \in L(X)$ tal que $R(P)=M$.

Observação 6. Se $M$ é complementado em $X$ então $M$ é fechado.

Definição 7. Sejam $M$ e $N$ subconjuntos de um espaço vetorial $X$. Definimos $M+N$ como sendo o conjunto $\{m+n: m \in M, n \in N\}$ ao qual chamaremos soma de $M$ com $N$. Se $M \cap N=\{0\}$ então a soma $M+N$ é dita uma soma direta interna em $X$ e é denotada por $M \oplus N$.

Proposição 8. ([8], p. 48) Sejam $X$ um espaço de Banach e $M$ um subespaço fechado de $X$. Então $M$ é complementado em $X$ se, e somente se, existe um subespaço fechado $N$ de $X$ tal que $X=M \oplus N$. 
Proposição 9. ([27], Proposição 21.3.4) Todo subespaço fechado de codimensão finita de um espaço de Banach $X$ é complementado em X.

Definição 10. Uma cisão de um espaço métrico $M$ é uma decomposição $M=A \cup B$, onde $A$ e $B$ são dois subconjuntos abertos disjuntos de $M$. A cisão $M=A \cup B$ diz-se trivial quando um dos abertos é vazio.

Definição 11. Um espaço métrico $M$ é dito conexo quando a única cisão possível de $M$ é a trivial.

Definição 12. Seja $M$ um espaço métrico. Dado $x \in M$, a componente conexa de $x$ em $M$ é a reunião $C_{x}$ de todos os subconjuntos conexos de $M$ que contém $x$.

Definição 13. Um caminho num espaço métrico $M$ é uma aplicação contínua $f:[0,1] \rightarrow$ $M$. Os pontos $a=f(0)$ e $b=f(1)$ são os extremos do caminho $f$.

Definição 14. Um espaço métrico $M$ é dito conexo por caminhos se quaisquer dois pontos de $M$ podem ser unidos por um caminho contido em $M$.

Proposição 15. ([19], Exemplo 17) Se $X \subset \mathbb{R}^{2}$ é um subconjunto enumerável do plano então $\mathbb{R}^{2}-X$ é conexo por caminhos.

Definição 16. Seja $X$ um espaço de Banach. Uma aplicação linear contínua $f: X \rightarrow \mathbb{R}$ é dita um funcional linear.

Definição 17. O dual $X^{*}$ de um espaço vetorial normado $X$ é o espaço (de Banach) dos funcionais lineares sobre $X$.

Proposição 18 (Teorema de Hahn-Banach). ([8], p. 17) Seja X um espaço vetorial sobre $\mathbb{R}($ ou $\mathbb{C})$. Seja $M$ um subespaço de $X$ e seja $g$ uma função a valores reais sobre $X$ com as seguintes propriedades:

(i) $g(x+y) \leq g(x)+g(y), \forall x, y \in X$;

(ii) $g(\alpha x)=|\alpha| g(x), \forall x \in X, \forall \alpha \in \mathbb{R}($ ou $\mathbb{C})$.

Se $f$ é um funcional linear sobre $M$ tal que $|f(m)| \leq g(m), \forall m \in M$, então existe um. funcional linear $F$ sobre $X$ tal que $F(x)=f(x), \forall x \in M$ e $|F(x)| \leq g(x), \forall x \in X$.

Definição 19. Dado um espaço vetorial $X$, definimos a aplicação natural, $J_{X}: X \rightarrow X^{* *}$, onde $X^{* *}$ é o espaço de Banach dos funcionais lineares sobre $X^{*}$, por

$$
\left(J_{X} x\right)\left(x^{*}\right)=x^{*}(x), \forall x^{*} \in X^{*}, \forall x \in X .
$$

Se a aplicação $J_{X}$ é sobrejetora então o espaço $X$ é dito reflexivo. 
Proposição 20. ([13]) Não existe um subespaço fechado de dimensão infinita e reflexivo de $C[0,1]$ que seja complementado em $C[0,1]$.

Definição 21. ([8], p.29) Uma seqüência $\left\{x_{n}\right\}$ em um espaço vetorial normado $X$ é dita fracamente convergente a $x \in X$ se $x^{*}\left(x_{n}\right) \rightarrow x^{*}(x), \forall x^{*} \in X^{*}$. Denotaremos $x_{n} \stackrel{w}{\rightarrow} x$.

Definição 22. Sejam $X$ e $Y$ espaços vetoriais normados e $T$ um operador de $X$ em $Y$. O operador $T$ é dito fracamente compacto se leva seqüencias limitadas em $X$ sobre seqüências que possuem uma subseqüência fracamente convergente em $Y$.

Definição 23. Seja $T: X \rightarrow Y$ um operador linear, onde $X$ e $Y$ são espaços vetoriais normados. O gráfico $G(T)$ de $T$ é o subconjunto $\{(x, T(x)): x \in X\}$ de $X \oplus Y$. Se $G(T)$ é fechado em $X \oplus Y$ então $T$ é dito um operador fechado.

Proposição 24. ([8], Observação II.1.3) Sejam $X$ e $Y$ espaços vetoriais normados e $T: X \rightarrow Y$ um operador linear.

(i) $T$ é fechado se, e somente se, para qualquer seqüência $\left\{x_{n}\right\}$ em $X$, com $x_{n} \rightarrow x$ e $T\left(x_{n}\right) \rightarrow y \in Y$, tem-se que $x \in D(T)$ e $y=T(x)$;

(ii) Se $T$ é fechado e injetor então $T^{-1}$ é fechado;

(iii) Se $T$ é fechado então $N(T)$ é um subespaço fechado.

Proposição 25 (Teorema da aplicação aberta). ([8], p. 45) Sejam X e Y espaços de Banach. Se $T: X \rightarrow Y$ é um operador linear fechado sobrejetor, então $T$ é uma aplicação aberta, isto é, T leva qualquer conjunto aberto sobre um conjunto aberto.

Proposição 26 (Teorema do gráfico fechado). ([8], p. 45) Sejam X e $Y$ espaços de Banach e $T: X \rightarrow Y$ um operador fechado. Então $T$ é contínuo.

Definição 27. Sejam $X$ e $Y$ espaços de Banach. Se $T \in L(X, Y)$ então o operador conjugado ou transposto de $T$ é o operador $T^{*}: Y^{*} \rightarrow X^{*}$, definido por $\left(T^{*}\left(y^{*}\right)\right)(x)=$ $y^{*}(T(x)), \forall y^{*} \in Y^{*}, \forall x \in X$.

Proposição 28. ([8], Teorema II.2.8) Sejam $X$ e Y espaços de Banach e $T: X \rightarrow Y$ um operador linear. Se $T$ é contínuo então $T^{*}$ também é contínuo e $\left\|T^{*}\right\|=\|T\|$.

Proposição 29. ([8], Teorema II.3.1) Sejam $X$ e $Y$ espaços de Banach e $T \in L(X, Y)$. Se $T^{*}$ possui uma inversa contínua então a imagem $R\left(T^{*}\right)$ de $T^{*}$ é fechada. 
Definição 30. Um conjunto $K$ em um espaço vetorial normado $X$ é ortogonal a um conjunto $F \subset X^{*}$ se $x^{*}(k)=0, \forall k \in K$ e $\forall x^{*} \in F$. O complemento ortogonal de $K$ em $X^{*}$, denotado por $K^{\perp}$, é o conjunto dos elementos em $X^{*}$ que são ortogonais a $K$.

Definição 31. Se $C$ é um subconjunto de $X^{*}$ então o complemento ortogonal de $C$ em $X$ é o conjunto ${ }^{\perp} C=\left\{x \in X: x^{*}(x)=0, \forall x^{*} \in C\right\}$.

Observação 32. ([8], p. 25) Seja $K$ um subconjunto de um espaço vetorial normado $X$ e $C$ um subconjunto de $X^{*}$. Então $K^{\perp}$ e ${ }^{\perp} C$ são subespaços fechados de $X^{*} \in X$, respectivamente. Além disso, $K^{\perp}=\bar{K}^{\perp} e^{\perp} C={ }^{\perp} \bar{C}$.

Proposição 33. ([8], Teorema 1.6.4) Seja $M$ um subespaço de um espaço vetorial normado X. Então

(i) $X^{*} / M^{\perp}$ é isométrico a $M^{*}$ pela aplicação $U: X^{*} / M^{\perp} \rightarrow M^{*}$ definida por $U\left(\left[x^{*}\right]\right)=$ $x_{M}^{*}$, onde $\left[x^{*}\right] \in X^{*} / M^{\perp}$ e $x_{M}^{*}$ é a restrição de $x^{*}$ a $M$;

(ii) Se $M$ é fechado (e então $X / M$ é um espaço vetorial normado) então $(X / M)^{*}$ é isométrico a $M^{\perp}$ pela aplicação $V$ definida por $\left(V\left(z^{*}\right)\right)(x)=z^{*}([x]), \forall z^{*} \in(X / M)^{*}, \forall x \in$ $X$.

Proposição 34. ([8], Teorema II.3.7) Sejam $X$ e $Y$ espaços de Banach e $T \in L(X, Y)$. Então

(i) $\overline{R(T)^{\perp}}=R(T)^{\perp}=N\left(T^{*}\right)$;

(ii) $\overline{R(T)}={ }^{\perp} N\left(T^{*}\right)$.

Em particular, $T$ possui imagem $R(T)$ densa em $Y$ se, e somente se, $T^{*}$ é injetor.

Proposição 35. ([8], Teorema II.3.9) Seja $T \in L(X, Y)$, onde $X$ e $Y$ são espaços de Banach. Se $T$ e $T^{*}$ possuem inversas contínuas, então $\left(T^{-1}\right)^{*}=\left(T^{*}\right)^{-1}$.

Proposição 36. ([8], Teorema II.4.4) Sejam X e $Y$ espaços vetoriais normados e T: $X \rightarrow Y$ um operador linear. Então

(i) $R\left(T^{*}\right)=X^{*}$ se, e somente se, $T$ possui uma inversa contínua;

(ii) Suponhamos que $X$ e $Y$ são espaços de Banach e que $T$ é um operador fechado. Então $R(T)=Y$ se, e somente se, $T^{*}$ possui uma inversa contínua.

Definição 37. Sejam $X$ e $Y$ espaços de Banach e $T: X \rightarrow Y$ um operador linear. $O$ operador $\widehat{T}$ induzido por $T: X \rightarrow Y$ é o operador de $X / N(T)$ em $Y$ definido por

$$
\widehat{T}([x])=T(x) \text {. }
$$


Note que $\widehat{T}$ é injetor, linear e possui a mesma imagem de $T$.

Proposição 38. ([8], Lema II.4.7) Sejam $X$ e $Y$ espaços de Banach e $T: X \rightarrow Y$ um operador linear. Suponhamos que o núcleo $N(T)$ de $T$ é fechado e seja $\widehat{T}$ o operador injetor induzido por T. Então

(i) $T$ é fechado se, e somente se, $\widehat{T}$ é fechado;

(ii) $T$ é contínuo se, e somente se, $\widehat{T}$ é contínuo; neste caso, $\|T\|=\|\widehat{T}\|$;

(iii) $\left\|(\widehat{T})^{*}\left(y^{*}\right)\right\|=\left\|T^{*}\left(y^{*}\right)\right\|, \forall y^{*} \in Y^{*}$.

Definição 39. Dois espaços de Banach $X$ e $Y$ são totalmente incomparáveis se não existem subespaços de dimensão infinita $X_{0}$ de $X \in Y_{o}$ de $Y$ tais que $X_{o}$ é isomorfo a $Y_{o}$.

Exemplo 40. ([20]) Os espaços $c_{o}(\mathbb{N})$ e $l_{p}(\mathbb{N}), 1 \leq p \leq \infty$ são totalmente incomparáveis entre si.

Definição 41. Sejam $X$ e $Y$ espaços de Banach. Um operador linear contínuo $T: X \rightarrow Y$ é dito estritamente singular se a restrição de $T$ a qualquer subespaço fechado de dimensão infinita de $X$ não é um isomorfismo sobre a imagem.

\section{Observação 42 .}

Se $X$ e $Y$ são totalmente incomparáveis é claro que todo operador linear contínuo de $X$ em $Y$ é estritamente singular. Porém, a recíproca não é verdadeira pois $l_{\infty}(\mathbb{N})$ e $c_{o}(\mathbb{N})$ não são totalmente incomparáveis e contudo, todo operador linear contínuo de $l_{\infty}(\mathbb{N})$ em $c_{o}(\mathbb{N})$ é estritamente singular.

De fato, seja $T: l_{\infty}(\mathbb{N}) \rightarrow c_{o}(\mathbb{N})$ um operador linear contínuo. Suponhamos que exista um subespaço de dimensão infinita $V$ em $l_{\infty}(\mathbb{N})$ tal que $T_{\left.\right|_{V}}$ é um isomorfismo sobre a imagem. Como $T(V)$ tem dimensão infinita então existe um subespaço $W$ de $T(V)$ isomorfo a $c_{o}(\mathbb{N})$ e existe uma projeção $P: c_{o}(\mathbb{N}) \rightarrow W([20])$. Seja $Z=T^{-1}(W)$. Temos que $Z$ é isomorfo a $c_{o}(\mathbb{N})$. Mas, de acordo com o resultado que veremos no Lema 175, temos que $Z$ é complementado em $l_{\infty}(\mathbb{N})$, um absurdo pois todo subespaço de dimensão infinita complementado em $l_{\infty}(\mathbb{N})$ é isomorfo a $l_{\infty}(\mathbb{N})([21])$.

Definição 43. Sejam $X$ e $Y$ espaços de Banach. Um operador $T: X \rightarrow Y$ é dito estritamente cosingular se não existe um subespaço fechado $N$ de codimensão infinita de $Y$ tal que $R(T)+N=Y$. 
Definição 44. S'ejam $X$ e $Y$ espaços de Banach. Um operador linear contínuo $K: X \rightarrow$ $Y$ é dito compacto se o conjunto $\overline{K\left(S_{X}\right)}$ é compacto em $Y$, onde $B_{X}$ é a bola unitária de $X$.

Proposição 45. ( [8], Teorema III.1.3) Sejam X e Y espaços de Banach. Todo operador $K: X \rightarrow Y$ compacto é estritamente singular.

Observação 46. Denotaremos por $S S(X, Y)$ o conjunto de todos os operadores estritamente singulares entre $X$ e $Y$ e por $K(X, Y)$ o conjunto de todos os operadores compactos de $X$ em $Y$. O teorema anterior mostra que $K(X, Y) \subset S S(X, Y)$. O Exemplo II.3.7 de [8] mostra que nem sempre temos $K(X, Y) \doteq S S(X, Y)$.

Definição 47. Sejam $X$ e $Y$ espaços de Banach. Um operador $S \in L(X, Y)$ é dito de posto finito se a sua imagem $R(S)$ é de dimensão finita, ou equivalentemente, se, admite uma representação finita da forma $S=\sum_{i=1}^{n} x_{i}^{*}\left(y_{i}\right)$, onde $x_{1}^{*}, \ldots, x_{n}^{*} \in X^{*}$ e $y_{1}, \ldots, y_{n} \in Y$. Nesse caso a dimensão de $R(S)$ é chamada de posto de $S$. A classe de todos os operadores de posto finito de $X$ em $Y$ é denotada por $\mathcal{F}(X, Y)$.

Proposição 48. ([8], Observações III.1.4) Sejam X e Y espaços de Banach e $T \in$ $L(X, Y)$.

(i) Se $T$ é de posto finito, então T é compacto;

(ii) $T$ é compacto se, e somente se, $T$ leva qualquer seqüência limitada em uma seqüência que possui uma subseqüência convergente;

(iii) Se $T$ é injetor e fechado então $T^{-1}$ é fechado.

Proposição 49. ([8], Teorema III.1.9) Sejam X e $Y$ espaços de Banach de dimensão infinita. Suponhamos que $T: X \rightarrow Y$ seja um operador linear contínuo tal que a restrição de $T$ a qualquer subespaço de $X$ de codimensão finita não é um isomorfismo sobre a imagem (este é o caso, em particular, se $R(T)$ não é fechada em $Y$ ). Então, para qualquer $\varepsilon>0$ existe um subespaço de dimensão infinita $M(\varepsilon) \subset X$ tal que a restrição $T_{\left.\right|_{M(\varepsilon)}}$ de $T$ a $M(\varepsilon)$ é compacto e $\left\|T_{\left.\right|_{M(\varepsilon)}}\right\| \leq \varepsilon$. No caso em que $R(T)$ não é fechada temos que o subespaço $M(\varepsilon)$ é fechado.

Proposição 50. ([8], Teorema III.2.5) Seja $K \in L(X, Y)$ um operador compacto, onde $X$ e $Y$ são espaço de Banach. Se $A \in L(Z, X)$ e $B \in L(Y, Z)$, onde $Z$ é um espaço de Banach, então temos que $K A$ e $B K$ também são operadores compactos. 
Proposição 51. ([8], Teorema II.2.5) Seja Z um espaço vetorial normado. Se $K \in$ $S S(X, Y)$ então para qualquer operador $A \in L(Z, X)$ temos que $K A \in S S(Z, Y)$. Analogamente, se $B \in L(Y, Z)$ então $B K \in S S(X, Y)$.

Proposição 52. ([9], Teorema II.8.1) Seja X um espaço de Banach e suponhamos que $A \in L(X)$ e $\|A\|<1$. Então $R(I-A)=X,(I-A)^{-1}$ existe e pertence a $L(X)$, e

$$
(I-A)^{-1}=I+A+A^{2}+\cdots+A^{n}+\cdots
$$

em $L(X)$. Além disso, $\left\|(I-A)^{-1}\right\| \leq \frac{1}{1-\|A\|}$.

Proposição 53. ([9], Proposição II.8.2) Sejam $X$ um espaço de Banach e $T \in L(X)$ um isomorfismo. Suponhamos que $B \in L(X)$ e que $\|T-B\|<\frac{1}{\left\|T^{-1}\right\|}$. Então B também é um isomorfismo e $B^{-1}(x)=\sum_{k=0}^{\infty}\left[T^{-1}(T-B)\right]^{k} T^{-1}(x), \forall x \in X$.

Proposição 54. ([24], Proposição A.3.4) Sejam $X$ e $Y$ espaços de Banach e $S \in L(X, Y)$. Se $Q$ denota a aplicação quociente canônica de $X$ sobre $X_{o}=X / N$, onde $N=N(S)$ denota o núcleo de $S$ e $J$ denota a inclusão canônica de $Y_{o}=\overline{R(S)}$ em $Y$ então existe uma fatorização

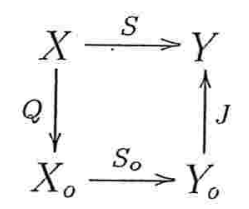

tal que $S_{o} \in L\left(X_{o}, Y_{o}\right)$ é injetor e $\overline{R\left(S_{o}\right)}=Y_{o}$. Além disso, $\left\|S_{o}\right\|=\|S\|$.

Proposição 55. ([24], Lema E.1.1) Sejam $X$ um espaço de Banach e $M$ um subespaço de $X \operatorname{com} M \neq X$. Então, dado $\varepsilon>0$ existe $x_{o} \in X$ tal que $\left\|x_{o}\right\|=1$ e $\left\|x-x_{o}\right\| \geq$ $\frac{1}{1+\varepsilon}, \forall x \in M$

Proposição 56. ([20], Lema 2.c.8) Sejam $M$ e $N$ subespaços de um espaço de Banach $X$ com $\operatorname{dim}(C)>\operatorname{dim}(B)$ (nesse caso, $\operatorname{dim}(B)<\infty)$. Então existe um $m \neq 0$ em $M$ tal que $\|m\|=d(m, N)=1$.

Definição 57. Um espaço de Banach $X$ é dito decomponível se ele contém um par de subespaços fechados de dimensão infinita $M, N$ tais que $X=M \oplus N$. Caso contrário, isto é, se não existem tais subespaços em $X$ então $X$ é dito indecomponível.

Definição 58. Um espaço de Banach $X$ é dito primo se $X$ é isomorfo a $M$, para qualquer subespaço complementado de dimensão infirita $M$ de $X$. 
O teorema abaixo resume algumas propriedades de um espaço de Banach construído por Gowers, W.T. e Maurey, B. que será utilizado no Capítulo 5. Veja (4.2), Teorema 13 e Teorema 16 de [12]

Teorema 59. Existe um espaço de Banach $Z$ com base de Schauder $\left\{z_{n}\right\}$ satisfazendo:

(i) $Z$ é indecomponivel;

(ii) $Z$ é primo;

(iii) Um subespaço $M$ de $Z$ é isomorfo a $Z$ se, e somente se, possui codimensão finita em Z.;

(iv) $O$ operador $S: Z \rightarrow Z$ dado por $S\left(a_{1}, a_{2}, \ldots\right)=\left(0, a_{1}, a_{2}, \ldots\right)$ é uma isometria sobre $Z$, onde $a_{1}, a_{2}, \ldots$ são as componentes com relação à base de Schauder $\left\{z_{n}\right\}$.

Definição 60. Sejam $X$ e $Y$ espaços de Banach e $T \in L(X, Y)$ um operador com imagem $R(T)$ fechada. Definimos o índice do núcleo e o índice de deficiência de $T$, respectivamente, por

$$
\alpha(T)=\operatorname{dim}(N(T)), \quad \beta(T)=\operatorname{dim}(Y / R(T)) .
$$

Definimos ainda $\bar{\beta}(T)=\operatorname{dim}(Y / \overline{R(T)})$.

Definição 61. Sejam $X$ e $Y$ espaços de Banach. Um operador $T \in L(X, Y)$ é dito semiFredholm superior se sua imagem $R(T)$ é fechada e $\alpha(T)<\infty$. O conjunto de todos os operadores semi-Fredholm superior é denotado por $\Phi_{+}(X, Y)$. Um operador $T \in L(X, Y)$ é dito semi-Fredholm inferior se $\beta(T)<\infty$ (neste caso, $R(T)$ é fechado pelo Corolário 73). O conjunto de todos os operadores semi-Fredholm inferior é denotado por $\Phi_{-}(X, Y)$. $O$ indice de um operador $T \in \Phi_{+}(X, Y) \cup \Phi_{-}(X, Y)$ é definido por

$$
i(T)=\alpha(T)-\beta(T) .
$$

$O$ indice é um número inteiro ou $\{ \pm \infty\}$. Um operador $T \in L(X, Y)$ é dito um operador de Fredholm se $i(T)<\infty$, ou equivalentemente, se $T \in \Phi_{+}(X, Y) \cap \Phi_{-}(X, Y)$. O conjunto de todos os operadores de Fredholm é denotado por $\Phi(X, Y)$.

Proposição 62. ([32], Corolário 2.3) Seja Y um espaço de Banach. Então (i) $L(Y, Z)=S S^{\prime}(Y, Z) \cup \Phi_{+}(Y, Z)$, para qualquer espaço de Banach $Z$ se, e somente se, todo subespaço fechado de $Y$ é indecomponivel;

(ii) $L(X, Y)=S C(X, Y) \cup \Phi_{-}(X, Y)$, para qualquer espaço de Banach $X$ se, e somente se, todo quociente de $Y$ é indecomponível. 
Proposição 63. ([26]) Sejam $X$ e $Y$ espaços de Banach. Se o operador $S \in L(Y, X)$ é tal que $\beta\left(I_{Y}-T S\right)=\infty$ então existe um subespaço fechado de codimensão infinita $N$ de $Y$ e um operador compacto $K \in L(Y)$ tais que $\pi_{N} K=\pi_{N}\left(I_{Y}-T S\right)$, onde $\pi_{N}$ denota a aplicação quociente canônica de $Y$ em $Y / N$, definida por $\pi_{N}(y)=[y], \forall y \in Y$. 


\section{Operadores de Fredholm}

Nesse capítulo abordaremos alguns resultados envolvendo os operadores de Fredholm (veja Definição 61). O objetivo é apresentar teoremas de perturbação de tais operadores por operadores estritamente singulares (veja Proposição 104) e também por operadores em um $\Phi$-ideal (veja Proposição 116). Esses teoremas de perturbação são ferramentas fundamentais para as demonstrações dos resultados parciais do Problema 1 (apresentado na Introdução) para espaços de Banach $X$ e $Y$ tais que todo operador linear contínuo de $X$ em $Y$ é estritamente singular e também para espaços de Banach $X$ e $Y$ tais que todo operador linear contínuo de $X$ em $Y$ é inessencial (veja Definição 151). Também apresentaremos algumas caracterizações dos operadores de Fredholm em termos de ideais de operadores que são fundamentais para simplificar a verificação de certos resultados que apresentaremos neste trabalho (veja por exemplo a Proposição 116). Iniciaremos este capítulo tratando de operadores com imagem fechada. Utilizaremos como principais referências [8] e [20].

\subsection{Módulo mínimo de um operador}

Lema 64. Sejam $X$ e $Y$ espaços de Banach e $T: D(T) \subset X \rightarrow Y$ um operador fechado. Então $T$ possui uma inversa contínua se, e somente se, $T$ é injetor e possui uma imagem fechada.

Prova. Suponhamos que $T$ possui uma inversa contínua. Então existe $k>0$ tal que $k\|x\| \leq\|T(x)\|, \forall x \in X$. Seja $\left\{y_{n}\right\}$ uma seqüencia em $R(T)$ tal que $y_{n} \rightarrow y$ em $Y$. 
Para cada $n \in \mathbb{N}$ temos que $y_{n}=T\left(x_{n}\right)$, onde $x_{n} \in X$. Logo, $k\left\|x_{n}-x_{m}\right\| \leq \| T\left(x_{n}\right)-$ $T\left(x_{m}\right) \|, \forall m, n \in \mathbb{N}$. Portanto $\left\{x_{n}\right\}$ é uma seqüência de Cauchy em $X$ e portanto $x_{n} \rightarrow x$, para algum $x \in X$. Isto implica que $T\left(x_{n}\right) \rightarrow T(x)=y$. Deste modo, $y \in R(T)$, implicando que $R(T)$ é um subespaço fechado de $Y$.

Reciprocamente, suponhamos que $T$ é injetor e $R(T)$ é um subespaço fechado de $Y$. Então $T^{-1}$ é um operador fechado que leva o espaço de Banach $R(T)$ no espaço de Banach $X$. De fato, suponhamos que $y_{n} \rightarrow y$ em $R(T)$ e $T^{-1}\left(y_{n}\right) \rightarrow x$ em $X$. Sendo $R(T)$ um subespaço fechado de $Y$ então $y \in R(T)$. Como $T$ é um operador fechado então $x_{n}=T^{-1}\left(y_{n}\right) \rightarrow x$ e $y_{n}=T\left(x_{n}\right) \rightarrow y$, implicando que $x \in D(T)=R\left(T^{-1}\right)$ e $y=T(x)$. Logo, obtemos que $y \in D\left(T^{-1}\right)=R(T)$ e $x=T^{-1}(y)$. Portanto $T^{-1}$ é fechado. Além disso $R(T)$ é um espaço de Banach pois todo espaço fechado contido num espaço completo é também completo. Então $T^{-1}$ é um operador linear contínuo pelo Teorema do gráfico fechado.

Proposição 65. Sejam $X$ e $Y$ espaços de Banach. Suponhamos que $T: X \rightarrow Y$ é um operador linear fechado. São equivalentes as seguintes afirmações:

(i) $R(T)$ é um subespaço fechado de $Y$;

(ii) $R\left(T^{*}\right)$ é um subespaço fechado de $X^{*}$;

(iii) $R\left(T^{*}\right)$ é o complemento ortogonal de $N(T)$; isto é, $R\left(T^{*}\right)=N(T)^{\perp}$;

(iv) $R(T)$ é o complemento ortogonal de $N\left(T^{*}\right)$; isto é, $R(T)={ }^{\perp} N\left(T^{*}\right)$.

Prova. (i) $\Leftrightarrow(i v)$.

Como $R(T)$ é um subespaço fechado de $Y$ então $\overline{R(T)}=R(T)$ e o resultado segue da Proposição 34-(ii), p. 10.

(i) $\Rightarrow$ (iii)

Seja $\widehat{T}$ o operador injetor induzido por $T$ (ver p. 10). Segue de (i) que $R(\widehat{T})=R(T)$ é um subespaço fechado de $Y$ e, como $\widehat{T}$ é injetor segue do Lema 64 que $\widehat{T}$ possui uma inversa contínua. Então, pela Proposição 36, p. 10, temos que $R\left((\widehat{T})^{*}\right)=(X / N(T))^{*}$. Como $N(T)$ é fechado então segue da Proposição 33-(ii) que $(X / N(T))^{*}$ é isométrico a $N(T)^{\perp}$ através da aplicação $V$ dada por $\left(V z^{*}\right)(x)=z^{*}([x]), \forall z^{*} \in(X / N(T))^{*}, \forall x \in X$. Deste modo,

$$
V\left(R(\widehat{T})^{*}\right)=N(T)^{\perp}
$$

Como $(\widehat{T})^{*}\left(y^{*}\right)[x]=T^{*}\left(y^{*}\right) x, \forall y^{*} \in Y^{*}$, então segue de (2.1) e da definição de $V$ que 
$R\left(T^{*}\right)=V\left(R(\widehat{T})^{*}\right)=N(T)^{\perp}$.

$(i i i) \Rightarrow(i i)$.

É imediato pois $N(T)^{\perp}$ é um subespaço fechado.

(ii) $\Rightarrow($ i $)$.

Seja $Y_{1}=\overline{R(T)}$ e seja $T_{1}: X \rightarrow Y_{1}$, onde $T_{1}=T$ é considerado como um operador linear no espaço de Banach $Y_{1} . T_{1}$ é obviamente um operador fechado. Para provar (i) mostraremos que $R\left(T_{1}\right)=Y_{1}=\overline{R(T)}$. Isto é equivalente a mostrar, pela. Proposição 36, p. 10, que $T_{1}^{*}$ possui uma inversa contínua. Como $\overline{R\left(T_{1}\right)}=Y_{1}$, então $T_{1}^{*}$ possui uma inversa. contínua, pela Proposição 36-(ii).

$(i v) \Rightarrow(i)$

Segue imediatamente da Observação 32, p. 10.

Sejam $X$ e $Y$ espaços de Banach. A cada operador linear $T: X \rightarrow Y$ com núcleo $N(T)$ fechado associamos um número $\gamma(T)$, que utilizaremos mais adiante em alguns resultados de perturbação de operadores de Fredholm. Obtemos tal número a partir das seguintes considerações:

Suponhamos que $T: X \rightarrow Y$ é um operador fechado, onde $X$ e $Y$ são espaços de Bana.ch. Seja $\widehat{T}$ o operador induzido por $T$ (ver Definição 37, p. 10). Então, pelo Lema 64 , temos que $R(T)=R(\widehat{T})$ é fechado se, e somente se, $\widehat{T}$ possui uma inversa contínua, ou equivalentemente, se

$$
\begin{aligned}
& 0<\inf \left(\frac{\|\widehat{T}([x])\|}{\|x\|}:[x] \in X / N(T), x \notin N(T)\right)= \\
& =\inf \left(\frac{\|T(x)\|}{d(x, N(T))}: x \in X-N(T)\right)=\frac{1}{\left\|(\widehat{T})^{-1}\right\|} .
\end{aligned}
$$

Definição 66. Sejam $X$ e $Y$ espaços de Banach e $T: D(T) \subset X \rightarrow Y$ um operador linear. Suponhamos que o núcleo $N(T)$ de $T$ é fechado. O módulo mínimo de $T$, denotado por $\gamma(T)$, é definido por $\gamma(T)=\inf \left(x \in D(T): \frac{\|T(x)\|}{d(x, N(T))}\right)$, onde $0 / 0$ é definido como $\infty$.

Exemplo 67. Cálculo do módulo mínimo de um operador definido no espaço de Banach $X=Y=l_{p}(\mathbb{N}), \quad 1 \leq p<\infty$.

Seja $\left\{\lambda_{k}\right\}$ uma seqüência limitada de números reais e seja $T: X \rightarrow Y$ o operador definido por $T\left(\left\{\alpha_{k}\right\}\right)=\left\{\lambda_{k} \alpha_{k}\right\}$. Para cada escalar $\lambda$ calcularemos $\gamma\left(T_{\lambda}\right)$, onde $T_{\lambda}=\lambda I-T$ 
e $I$ é o operador identidade em $l_{p}$. Seja $E_{\lambda}=\left\{k: \lambda \neq \lambda_{k}\right\}$. Então $N\left(T_{\lambda}\right)=\left\{\left(\beta_{1}, \beta_{2}, \ldots\right) \in\right.$ $l_{p}: \beta_{k}$ é arbitrário-se $k \notin E_{\lambda}$ e $\beta_{k}=0$ se $\left.k \in E_{\lambda}\right\}$. Para simplificar, calcularemos $\gamma\left(T_{\lambda}\right)$ quando $X=l_{1}$. O cálculo para $1<p<\infty$ é essencialmente o mesmo. Seja $x=\left\{\eta_{k}\right\}$ em $X$. Então para $y=\left\{\beta_{k}\right\} \in N\left(T_{\lambda}\right)$,

$$
\|x-y\|=\sum_{k \notin E_{\lambda}}\left|\eta_{k}-\beta_{k}\right|+\sum_{k \in E_{\lambda}}\left|\eta_{k}\right| .
$$

Como os $\beta_{k}$ podem ser escolhidos como sendo os $\eta_{k}$ para $k \notin E_{\lambda}$ (veja na definição do núcleo $N\left(T_{\lambda}\right)$ de $T_{\lambda}$ que nesse caso $\beta_{k}$ é arbitrário), segue que $\|[x]\|=d\left(x, N\left(T_{\lambda}\right)\right)=$ $\sum_{k \in E_{\lambda}}\left|\eta_{k}\right|$. Deste modo,

$$
\frac{\left\|T_{\lambda}(x)\right\|}{\|[x]\|}=\frac{\sum_{k \in E_{\lambda}}\left|\lambda-\lambda_{k}\right|\left|\eta_{k}\right|}{\sum_{k \in E_{\lambda}}\left|\eta_{k}\right|} \geq \inf \left\{\left|\lambda-\lambda_{k}\right|: k \in E_{\lambda}\right\},
$$

implicando que

$$
\gamma\left(T_{\lambda}\right) \geq \inf _{k \in E_{\lambda}}\left|\lambda-\lambda_{k}\right|=m>0 .
$$

Por outro lado, dado $\varepsilon>0$ existe um $i \in E_{\lambda}$ tal que $\left|\lambda-\lambda_{i}\right|<m+\varepsilon$. Seja $u_{i}$ o elemento de $l_{1}$ cujas componentes são todas nulas, exceto o i-ésimo termo que é 1. Então por (2.2) e (2.3),

$$
m \leq \gamma\left(T_{\lambda}\right) \leq \frac{\left\|T_{\lambda}\left(u_{i}\right)\right\|}{\left\|\left[u_{i}\right]\right\|}=\left|\lambda-\lambda_{i}\right|<m+\varepsilon .
$$

Desde que $\varepsilon>0$ é arbitrário, $\inf \left\{\left|\lambda-\lambda_{k}\right|: k \in E_{\lambda}\right\}=\gamma(\lambda I-T)$.

Proposição 68. Sejam $X$ e $Y$ espaços de Banach e $T: X \rightarrow Y$ um operador fechado. Então $R(T)$ é fechada se, e somente se, $\gamma(T)>0$.

Prova. Seja $\widehat{T}: X / N(T) \rightarrow Y$ o operador induzido por $T$. Temos, pelo Lema 64 , que $R(T)=R(\widehat{T})$ é fechada se, e somente se, $\widehat{T}$ possui uma inversa contínua, ou equivalentemente se

$$
\begin{aligned}
& 0<\inf \left(\frac{\|\widehat{T}([x])\|}{\|x\|}:[x] \in X / N(T), x \notin N(T)\right)= \\
& =\inf \left(\frac{\|T(x)\|}{d(x, N(T))}: x \in X-N(T)\right)=\frac{1}{\left\|(\widehat{T})^{-1}\right\|} .
\end{aligned}
$$

Proposição 69. Sejam $X$ e $Y$ espaços de Banach e $T: X \rightarrow Y$ um operador linear. Se $\gamma(T)>0$ e $R(T)$ é um subespaço fechado então $T$ é um operador fechado. 
Prova. Seja $\widehat{T}: X / N(T) \rightarrow Y$ o operador induzido por $T$. Então a inversa $(\widehat{T})^{-1}$ de $\widehat{T}$ é um operador contínuo cujo domínio é um subespaço fechado de $Y$, pois $\gamma(T)>0$. Segue daí que $\frac{1}{\left\|\widehat{T}^{-1}\right\|}>0$, implicando que $(\widehat{T})^{-1}$ é um operador contínuo. Desse modo, $(\widehat{T})^{-1}$ é um operador fechado e assim $\widehat{T}$ também é fechado pela Proposição 48-(iii), p. 12. Então $T$ é fechado pelo Lema 38, p. 11.

\section{Observação 70 .}

As Proposições 68 e 69 acima mostram que se $X$ e $Y$ são espaços de Banach e $T$ : $X \rightarrow Y$ é um operador linear então quaisquer duas das seguintes informações combinadas implica a outra:

(i) $T$ é um operador fechado;

(ii) $R(T)$ é um subespaço fechado de $Y$;

(iii) $\gamma(T)>0$.

Lema 71. Seja $Y$ um espaço de Banach. Suponhamos que $M$ e $N$ são subespaços fechados de $Y$ tais que $Y=M \oplus N$. Então $Y^{*}=M^{\perp}: \oplus N^{\perp}$.

Prova. Seja $y^{*} \in M^{\perp} \cap N^{\perp}$. Então $y^{*}(m)=0, \forall m \in M$ e $y^{*}(n)=0, \forall n \in N$. Como $Y=M \oplus N$ então $y^{*}(y)=0, \forall y \in Y$ e, portanto, $y^{*}=0$. Por outro lado, existe uma projeção $P$ de $Y$ sobre $M \operatorname{com} N(P)=N$. Dado $y^{*} \in Y^{*}$, seja $y_{1}^{*}=y^{*} P$ e seja $y_{2}^{*}=y^{*}\left({ }_{Y} I-P\right)$. Então $y^{*}=y_{1}^{*}+y_{2}^{*}$, onde $y_{1}^{*} \in N^{\perp}$ e $y_{2}^{*} \in M^{\perp}$.

Proposição 72. Sejam $X$ e $Y$ espaços de Banach e $T: D(T) \subset X \rightarrow Y$ um operador fechado. Suponhamos que $N$ é um subespaço fechado de $Y$ tal que $R(T) \oplus N$ é fechado. Então $R(T)$ é fechada. Se $D(T)$ é denso em $X$ então $T^{*}\left(Y^{*}\right)=T^{*}\left(N^{\perp}\right)$.

Prova. Consideremos o operador linear $T_{0}: D\left(T_{o}\right)=D(T) \oplus N \rightarrow Y$ definido por $T_{o}(x, n)=T(x)+n, \forall x \in X, \forall n \in N$. Suponhamos que $\left(x_{k}, n_{k}\right) \rightarrow(x, y) \in X \oplus Y \mathrm{e}$ $T_{o}\left(x_{k}, n_{k}\right) \rightarrow z$. Então $x_{k} \rightarrow x, n_{k} \rightarrow y$ e $T\left(x_{k}\right)+n_{k} \rightarrow z$. Como $T$ é fechado então $x \in D(T)$ e $T(x)=z-y$. Além disso temos que $y \in N$ pois $N$ é fechado por hipótese. Deste modo, $(x, y) \in D(T) \oplus N=D\left(T_{o}\right)$ e $T_{o}(x, y)=T(x)+y=z$. Portanto, o operador $T_{o}$ é fechado.

Por hipótese, $R\left(T_{o}\right)=R(T) \oplus N$ é fechada. Pela Observação 70 temos que $\gamma\left(T_{o}\right)>0$. Para provar que $R(T)$ é fechado mostraremos que $\gamma(T)>0$. Desde que $R(T) \cap N=\{0\}$ 
temos que $N\left(T_{0}\right)=N(T) \oplus\{0\}$. Deste modo, dado $x \in D(T)$, temos que

$$
\|T(x)\|=\left\|T_{o}(x, 0)\right\| \geq \gamma\left(T_{o}\right)\left(d\left((x, 0), N\left(T_{o}\right)\right)\right)=\gamma\left(T_{o}\right) d(x, N(T)) .
$$

Então $\frac{\|T(x)\|}{d(x, N(T))} \geq \gamma\left(T_{o}\right)$, implicando que $\gamma(T) \geq \gamma\left(T_{o}\right)>0$.

Suponhamos que $D(T)$ é denso em $X$ e seja $y^{*} \in D\left(T^{*}\right)$. Seja $Y_{1}=R(T) \oplus N$. Então, pelo Lema $71, Y_{1}^{*}=R(T)^{\perp} \oplus N^{\perp}$. Deste modo, se $y_{k}^{*}=y_{\left.\right|_{1}}^{*}$ (onde $y_{\left.\right|_{Y_{1}}}$ é a restrição de $y^{*}$ a. $Y_{1}$ ) então existem $y_{1}^{*} \in R(T)^{\perp}$ e $y_{2}^{*} \in N^{\perp}$ tais que $y_{k}^{*}=y_{1}^{*}+y_{2}^{*}$. Seja $v^{*}$ uma extensão linear contínua de $y_{1}^{*}$ a todo o espaço $Y$. Então $v^{*}$ está em $R(T)^{\perp}=N\left(T^{*}\right)$, pela Proposição 65 . Então $T^{*}\left(y^{*}\right)=T^{*}\left(y^{*}-v^{*}\right)$. Além disso, $y^{*}-v^{*} \in N^{\perp}$, desde que $y^{*}-v^{*}=y_{2}^{*}$ sobre $N$.

Corolário 73. Sejam $X$ e $Y$ espaços de Banach e $T: X \rightarrow Y$ um operador fechado. Se $R(T)$ possui codimensão finita em $Y$ então $R(T)$ é um subespaço fechado.

Prova. Como $R(T)$ é de codimensão finita então $Y=R(T) \oplus N$, onde $N$ é algum subespaço de dimensão finita de $Y$. Como subespaços de dimensão finita são fechados então segue da Proposição 72 que $R(T)$ é fechado.

Proposição 74. Sejam $X$ e $Y$ espaços de Banach e $T \in L(X, Y)$. Se $\gamma(T)>0$ então $\gamma\left(T^{*}\right)=\gamma(T)$ e $T^{*}$ possui imagem fechada.

Prova. Segue das Proposições 34 e 33 que $Y^{*} / N\left(T^{*}\right)=Y^{*} / R(T)^{\perp}$ é isométrico a $R(T)^{*}$ através da isometria $\left[y^{*}\right] \rightarrow y_{R}^{*}$, onde $y_{R}^{*}$ é a restrição de $y^{*}$ a $R(T)$. Em particular,

$$
\left\|\left[y^{*}\right]\right\|=\left\|y_{R}^{*}\right\| .
$$

É claro que para o operador $\widehat{T}$ induzido por $T$ temos que $\left\|(\widehat{T})^{*}\left(y^{*}\right)\right\|=\left\|(\widehat{T})^{*}\left(y_{R}^{*}\right)\right\|$. Deste modo, para $y^{*} \in Y^{*}$ temos, pela Proposição 38 , que

$$
\left\|(\widehat{T})^{*}\left(y_{R}^{*}\right)\right\|=\left\|T^{*}\left(y^{*}\right)\right\| .
$$

Além disso, pelas Proposições 35 e 28 temos que

$$
\left\|\left((\widehat{T})^{*}\right)^{-1}\right\|=\left\|\left(\widehat{T}^{-1}\right)^{*}\right\|=\left\|\widehat{T}^{-1}\right\| .
$$


Finalmente, segue de (2.4), (2.5) e (2.6) que

$$
\begin{aligned}
\gamma\left(T^{*}\right) & =\inf \left\{\left\|T^{*}\left(y^{*}\right)\right\| /\left\|\left[y^{*}\right]\right\|:\left[y^{*}\right] \in Y^{*} / N(T)\right\}= \\
& =\inf \left\{\left\|(\widehat{T})^{*}\left(y_{R}^{*}\right)\right\| /\left\|y_{R}^{*}\right\|: y_{R}^{*} \in R(T)^{*}\right\}= \\
& =\frac{1}{\left\|\left((\widehat{T})^{*}\right)^{-1}\right\|}=\frac{1}{\left\|\widehat{T}^{-1}\right\|}=\gamma(T) .
\end{aligned}
$$

$\mathrm{E}$ isso conclui a prova.

\subsection{Operadores de Fredholm}

Lembremos que dados dois espaços de Banach $X$ e $Y$ então um operador $T \in L(X, Y)$ é dito um operador de Fredholm se $i(T)<\infty$.

Exemplo 75. Seja $k>0$. O operador linear $T: c_{o}(\mathbb{N}) \rightarrow c_{o}(\mathbb{N})$ definido por

$$
T\left(a_{1}, a_{2}, a_{3}, \ldots\right)=\left(a_{k+1}, a_{k+2}, a_{k+3}, \ldots\right)
$$

é um operador de Fredholm de indice $k>0$.

O operador $T$ é obviamente contínuo e sobrejetor. Portanto, segue que $R(T)=Y$ e, deste modo, $Y / R(T)=\{0\}$ implicando que $\beta(T)=0$. Temos também que $N(T)=$ $\left\{\left(a_{1}, a_{2}, a_{3}, \ldots\right) \in c_{o}:\left(a_{k+1}, a_{k+2}, \ldots\right)=(0,0,0, \ldots)\right\}=\left\{a_{1}(1,0,0, \ldots)+a_{2}(0,1,0,0, \ldots)+\right.$ $\left.\cdots+a_{k}(0,0, \ldots, 0,1,0, \ldots): a_{1}, \ldots, a_{k} \in \mathbb{R}\right\}$. Portanto, $\alpha(T)=k$. Assim, $i(T)=k$.

Observação 76 .

Sejam $X$ e $Y$ espaços de Banach. Então $T: X \rightarrow Y$ é um operador de Fredholm se, e somente se, existem subespaços $X_{1}$ e $B$ de $X, Y_{1}$ e $C$ de $Y, \operatorname{com} \operatorname{dim}(B)<\infty$ e $\operatorname{dim}(C)<\infty$, tais que $X=X_{1} \oplus B, Y=Y_{1} \oplus C, T_{\left.\right|_{B}}=0$ e a restrição $T_{\left.\right|_{X_{1}}}$ de $T$ a $X_{1}$ é um isomorfismo sobre $Y_{1}$. Com esta notação temos que $\alpha(T)=\operatorname{dim}(B)$ e $\beta(T)=\operatorname{dim}(C)$. Proposição 77. Sejam $X$ e $Y$ espaços vetoriais normados e $T: X \rightarrow Y$ um operador linear. Então

(i) $\alpha\left(T^{*}\right)=\bar{\beta}(T)$. Em particular, se $R(T)$ é fechado então $\alpha\left(T^{*}\right)=\beta(T)$;

(ii) Se $X$ e $Y$ são completos e $T$ é um operador fechado com imagem fechada então $\alpha(T)=\beta\left(T^{*}\right)$. Se, além disso, o operador $T$ possui um indice então $T^{*}$ também possui $u m$ indice e $i(T)=-i\left(T^{*}\right)$. 
Prova. (i) Pelas Proposições 33 e 34 (p. 10) temos que

$$
\begin{aligned}
\operatorname{dim}(Y / \overline{R(T)}) & =\operatorname{dim}\left((Y / \overline{R(T)})^{*}\right)=\operatorname{dim}\left(R(T)^{\perp}\right) \\
& =\operatorname{dim}\left(N\left(T^{*}\right)\right)=\alpha\left(T^{*}\right) .
\end{aligned}
$$

Assim, $\beta(T)=\alpha\left(T^{*}\right)$ quando a imagem $R(T)$ de $T$ é um subespaço fechado.

(ii) Pelas Proposições 65 e 33 temos que

$$
\begin{aligned}
\beta\left(T^{*}\right) & =\operatorname{dim}\left(X^{*} / R\left(T^{*}\right)\right)=\operatorname{dim}\left(X^{*} / N(T)^{\perp}\right)=\operatorname{dim}\left(N(T)^{*}\right) \\
& =\operatorname{dim}(N(T))=\alpha(T) .
\end{aligned}
$$

Portanto, $\beta\left(T^{*}\right)=\alpha(T)$, implicando que

$$
i(T)=\alpha(T)-\beta(T)=\beta\left(T^{*}\right)-\alpha\left(T^{*}\right)=-i\left(T^{*}\right) .
$$

Lema 78. Sejam $X$ um espaço de Banach e $M$ um subespaço fechado de codimensão finita em $X$. Então

(i) Para qualquer subespaço $V$ de $X$ existe um subespaço de dimensão finita $N$ contido em $V$ tal que $\bar{V}=(\bar{V} \cap M) \oplus N$;

(ii) Se $V$ é denso em $X$ então $V \cap M$ é denso em $M$.

Prova. (i) A dimensão de $\bar{V} /(\bar{V} \cap M)$ não excede a dimensão do espaço de dimensão finita $X / M$, desde que o operador linear $\eta$ de $\bar{V} / \bar{V} \cap M$ a $X / M$ definido por $\eta(x+\bar{V} \cap M)=$ $x+M, \forall x \in X$, é injetor. Então existe um subespaço de dimensão finita $W$ de $\bar{V}$ tal que

$$
\bar{V}=(\bar{V} \cap M) \oplus W
$$

Além disso, existe uma projeção $P$ de $\bar{V}$ sobre $W \operatorname{com} P(\bar{V} \cap M)=\{0\}$. Como $P(V)$ é de dimensão finita e $P$ é contínuo então segue que

$$
W=P(\bar{V}) \subset \overline{P(V)}=P(V) .
$$

Seja $\left\{w_{1}, \ldots, w_{n}\right\}$ uma base algébrica para $W$. Então, por (2.8), segue que existem elementos $v_{1}, \ldots, v_{n}$ em $V$ tais que $P\left(v_{i}\right)=w_{i}, \quad 1 \leq i \leq n$. Como os vetores $w_{1}, \ldots, w_{n}$ são linearmente independentes então o espaço $N=\left[v_{1}, \ldots, v_{n}\right]$ é um subespaço de dimensão $n$ de $V$ e $\{0\}=N \cap(M \cap \bar{V})$. Por (2.7) temos que

$$
\operatorname{dim}(\bar{V} / M \cap \bar{V})=\operatorname{dim}(W)=\operatorname{dim}(N) .
$$


Então $\bar{V}=(M \cap \bar{V}) \oplus N$.

(ii) Suponhamos que $\bar{V}=X$. Então, por-(i), temos que $X=M \oplus N$, onde $N$ é um subespaço de dimensão finita de $V$. Desta maneira $V=(M \cap V) \oplus N$. Temos também que $M$ é isomorfo a $X / N$ através da aplicação $\eta$, onde $\eta(m)=[m]$. Então podemos escrever

$$
M \stackrel{\eta}{\rightarrow} X / N \supset V / N=\frac{(M \cap V) \oplus N}{N} \stackrel{\eta^{-1}}{\rightarrow} M \cap V .
$$

Desde que $V$ é denso em $X$ temos que $V / N$ é denso em $X / N$. Notando que $\eta(M \cap V)=$ $V / N$ segue de (2.9) e da continuidade de $\eta$ e $\eta^{-1}$ que $M \cap V=\eta^{-1} \eta(M \cap V)$ é denso em $M$.

Lema 79. Sejam $X$ e $Y$ espaços de Banach e seja $T: X \rightarrow Y$ um operador fechado com imagem fechada. Se $M$ é um subespaço (não necessariamente fechado) de $X$ tal que $M+N(T)$ é fechado, então $T(M)$ é fechado. Em particular, se $M$ é fechado e $N(T)$ é de dimensão finita, então $T(M)$ é fechado.

Prova. Seja $T_{1}$ a restrição do operador $T$ ao subespaço $M+N(T)$. Então $T_{1}$ é fechado e $N\left(T_{1}\right)=N(T)$. Segue que $\gamma\left(T_{1}\right) \geq \gamma(T)>0$, onde $\gamma\left(T_{1}\right)$ e $\gamma(T)$ denotam, respectivamente, os módulos mínimos de $T_{1}$ e de $T$. Deste modo, segue da Observação 70 que $T_{1}$ possui imagem fechada, isto é, $T(M)=T_{1}(M+N(T))$ é fechado.

Proposição 80. Sejam $X$ e $Y$ espaços de Banach e $T: X \rightarrow Y$ um operador linear fechado com imagem fechada tal que $\alpha(T)<\infty$. Suponhamos que $B$ é um operador linear cujo domínio é um espaço vetorial normado $Z$ e cuja imagem está contida em $X$. Temos que

(i) Se B é um operador fechado então TB é também um operador fechado;

(ii) Se B é um operador fechado com imagem fechada então TB também é um operador fechado com imagem fechada;

(iii) Suponhamos que $Z$ é um espaço de Banach. Se T e B são operadores de Fredholm então TB é um operador de Fredholm e $i(T B)=i(T)+i(B)$.

Prova. (i) Suponhamos que $z_{n} \rightarrow z$ em $Z$ e $(T B)\left(z_{n}\right) \rightarrow y$ em $Y$. Como $\gamma(T)>0$ segue que a seqüência $\left\{\left[B\left(z_{n}\right)\right]\right\}$ em $X / N(T)$ é uma seqüência de Cauchy. Deste modo existe $[x] \in X / N(T)$ tal que $\left[B\left(z_{n}\right)\right] \rightarrow[x]$. Isto implica a existência de uma seqüência $\left\{x_{n}\right\}$ em $N(T)$ tal que $B\left(z_{n}\right)+x_{n} \rightarrow x$. Mostremos primeiro que a seqüência $\left\{x_{n}\right\}$ é limitada. 
Suponhamos que a seqüência $\left\{x_{n}\right\}$ é ilimitada. Então existe uma subseqüência $\left\{x_{n^{\prime}}\right\}$ de $\left\{x_{n}\right\}$ tal que

$$
\left\|x_{n^{\prime}}\right\| \rightarrow \infty \text { e } \frac{B\left(z_{n^{\prime}}\right)+x_{n^{\prime}}}{\left\|x_{n^{\prime}}\right\|} \rightarrow 0 .
$$

Como $\left\{x_{n^{\prime}} /\left\|x_{n^{\prime}}\right\|\right\}$ é uma seqüência limitada no espaço de dimensão finita $N(T)$ então existe uma subseqüência. $\left\{x_{n^{\prime \prime}}\right\}$ de $\left\{x_{n^{\prime}}\right\}$ e $v \in N(T)$ tal que $x_{n^{\prime \prime}} /\left\|x_{n^{\prime \prime}}\right\| \rightarrow v$. Segue que

$$
\frac{B\left(z_{n^{\prime \prime}}\right)}{\left\|x_{n^{\prime \prime}}\right\|} \rightarrow-v \text { e } \frac{z_{n^{\prime \prime}}}{\left\|x_{n^{\prime \prime}}\right\|} \rightarrow 0 .
$$

Desde que $B$ é um operador fechado então temos que $v=B(0)$, o que contradiz $\|v\|=1$. Deste modo $\left\{x_{n}\right\}$ é uma seqüência limitada em $N(T)$. Conseqüentemente existe uma subseqüência $\left\{x_{n^{\prime}}\right\}$ de $\left\{x_{n}\right\}$ e $w \in N(T)$ tal que $x_{n^{\prime}} \rightarrow w$. Então $B\left(z_{n^{\prime}}\right) \rightarrow x-w$. Como $z_{n^{\prime}} \rightarrow z$ e $B$ é fechado então segue que $z \in D(B)$ e $B\left(z_{n^{\prime}}\right) \rightarrow x-w=B(z)$. Como $T$ é um operador fechado e $(T B)\left(z_{n^{\prime}}\right) \rightarrow y$ então $B(z) \in D(T)$ e $(T B)(z)=y$. Portanto, $T B$ é um operador fechado.

(ii) Se $B(Z)$ é um subespaço fechado então $R(T B)=T B(Z)$ é fechado pelo Lema 79 .

(iii) O operador linear $\eta: N(T B) / N(B) \rightarrow R(B) \cap N(T)$ definido por $\eta([x])=B(x)$ é injetor e sobrejetor. Então para $N_{1}=R(B) \cap N(T)$ temos que

$$
\alpha(T B)=\alpha(B)+n_{1}, \text { onde } n_{1}=\operatorname{dim}\left(N_{1}\right)
$$

Seja $N_{2}$ um subespaço de $N(T)$ tal que $N(T)=N_{1} \oplus N_{2}$. Então

$$
\alpha(T)=n_{1}+n_{2}, \text { onde } n_{2}=\operatorname{dim}\left(N_{2}\right)
$$

Suponhamos que $B(x) \in R(B) \cap N_{2}$. Como $B(x) \in N_{2} \subset N(T)$ então $B(x) \in$ $N_{1} \cap N_{2}=\{0\}$, implicando que $R(B) \cap N_{2}=\{0\}$.

Como $\beta(B)$ e $\operatorname{dim}\left(N_{2}\right)$ são finitos então $R(B)$ e $R(B) \oplus N_{2}$ são subespaços fechados pelo Corolário 73 e pela Proposição 3. Segue do Lema 78 que

$$
R(B) \oplus N_{2} \oplus N_{3}=X,
$$

onde $N_{3}$ é algum subespaço de dimensão finita de $X$. Então

$$
\beta(B)=n_{2}+n_{3}, \text { onde } n_{3}=\operatorname{dim}\left(N_{3}\right)
$$


Temos que $N(T)=N_{1} \oplus N_{2} \subset R(B) \oplus N_{2}$. Este fato juntamente com (2.12) implica. que $T$ é injetor sobre $N_{3}$ e

$$
T(X)=T(R(B)) \oplus T\left(N_{3}\right)
$$

(Em geral, se $X=U \oplus V$, onde $N(T) \subset U$ e $V$ é um subespaço de $X$, então $T$ é injetor sobre $V$ e $T(X)=T(U) \oplus T(V)$ ). Segue de (2.14) e do fato de que $T$ é injetor sobre todo o subespaço $N_{3}$ que

$$
\beta(T B)=\beta(T)+\operatorname{dim}\left(T\left(N_{3}\right)\right)=\beta(T)+n_{3} .
$$

De (2.10), (2.15), (2.11) e (2.13) obtemos que

$$
\begin{aligned}
i(T B) & =\alpha(B)+n_{1}-\beta(T)-n_{3}= \\
& =\alpha(B)+n_{1}+n_{2}-\beta(T)-n_{2}-n_{3} \\
& =\alpha(B)-\beta(B)+\alpha(T)-\beta(T)=i(T)+i(B)
\end{aligned}
$$

\subsection{Ideais de operadores lineares}

Nesta seção faremos algumas considerações básicas sobre ideais de operadores que utilizaremos no decorrer da demonstração do Teorema 149 que apresentaremos no capítulo 6 e também para obter caracterizações dos operadores de Fredholm conforme veremos na seção 2.5 .

Lembremos que $L$ denota a classe de todos os operadores lineares contínuos entre espaços de Banach arbitrários.

Definição 81. Um ideal de operadores $\mathcal{U}$ é uma sub-classe de $L$ tal que dados espaços de Banach $X$ e $Y$ as componentes

$$
\mathcal{U}(X, Y)=\mathcal{U} \cap L(X, Y)
$$

satisfazem as seguintes condições:

1. $I_{E} \in \mathcal{U}$, onde $E$ denota o espaço de Banach de dimensão 1; 
2. Se $S_{1}, S_{2} \in \mathcal{U}(X, Y)$ então $S_{1}+S_{2} \in \mathcal{U}(X, Y)$;

3. Sejam $X_{o}$ e $Y_{o}$ espaços de Banach. Se $T \in L\left(X_{0}, X\right), \quad S \in \mathcal{U}(X, Y)$ e $R \in L\left(Y, Y_{o}\right)$ então $R S T \in \mathcal{U}\left(X_{0}, Y_{0}\right)$.

Se $X=Y$ denotaremos a componente $\mathcal{U}(X, X)$ por $\mathcal{U}(X)$.

Definição 82. Sejam $X$ e $Y$ espaços de Banach. Um operador $S \in L(X, Y)$ é dito aproximável se existem $S_{1}, S_{2}, S_{3}, \ldots \in \mathcal{F}(X, Y)$ tais que $\lim _{n \rightarrow \infty}\left\|S-S_{n}\right\|=0$. Denotaremos por $\mathcal{A P}(X, Y)$ a classe de todos os operadores aproximáveis de $X$ em $Y$ e por $\mathcal{A P}$ a classe de todos os operadores aproximáveis.

Proposição 83. A classe $\mathcal{A P}$ de todos os operadores aproximáveis é um ideal de operadores.

Prova. Obviamente, $I_{E} \in \mathcal{A P}$, onde $E$ denota o espaço de Banach de dimensão 1.

Sejam $X$ e $Y$ espaços de Banach e $S_{1}, S_{2} \in \mathcal{A} \mathcal{P}$. Então existem operadores

$$
S_{1}^{1}, S_{1}^{2}, \ldots, S_{1}^{n}, \ldots, S_{2}^{1}, S_{2}^{2}, \ldots, S_{2}^{n}, \ldots \in \mathcal{F}(X, Y)
$$

tais que dado $\varepsilon>0$ existem $n_{o}, n_{1} \in \mathbb{N}$ tais que $\left\|S_{1}-S_{1}^{n}\right\|<\frac{\varepsilon}{2}, \forall n \geq n_{o}$ e $\left\|S_{2}-S_{2}^{n}\right\|<$ $\frac{\varepsilon}{2}, \forall n \geq n_{1}$. Temos que $S_{1}^{i}+S_{2}^{j} \in \mathcal{F}(X, Y), \forall i, j \in \mathbb{N}$. Consideremos a seqüência $\left\{S_{1}^{i}+S_{2}^{i}\right\}$ em $\mathcal{F}(X, Y)$. Seja $n_{3}=\max \left\{n_{o}, n_{1}\right\} \in \mathbb{N}$. Então

$$
\left\|S_{1}+S_{2}-\left(S_{1}^{i}-S_{2}^{i}\right)\right\| \leq\left\|S_{1}-S_{1}^{i}\right\|+\left\|S_{2}-S_{2}^{i}\right\|<\frac{\varepsilon}{2}+\frac{\varepsilon}{2}=\varepsilon, \forall n \geq n_{3} .
$$

Logo, temos que $S_{1}+S_{2} \in \mathcal{A P}(X, Y)$.

Finalmente, sejam $X_{o}$ e $Y_{o}$ espaços de Banach, $T \in L\left(X_{o}, X\right), S \in \mathcal{A} \mathcal{P}(X, Y)$ e $R \in$ $L\left(Y, Y_{0}\right)$. Então existe uma seqüencia de operadores $\left\{S_{i}\right\}$ em $\mathcal{F}(X, Y)$ tais que dado $\varepsilon>$ $\theta$ existe $n_{o} \in \mathbb{N}$ tal que $\left\|S-S_{i}\right\|<\frac{\varepsilon}{\|R\|\|T\|}, \forall i \geq n_{o}$. Consideremos a seqüencia de operadores $\left\{R S_{i} T\right\}$ em $\mathcal{F}\left(X_{o}, Y_{o}\right)$. Temos que

$$
\left\|R S T-R S_{i} T\right\| \leq\|R\|\left\|S-S_{i}\right\|\|T\|<\varepsilon, \forall n \geq n_{i} .
$$

Portanto, $\mathcal{A P}$ é um ideal de operadores.

Definição 84. Sejam $X$ e $Y$ espaços de Banach. Um operador $S \in L(X, Y)$ é dito nuclear se existem $x_{1}^{*}, x_{2}^{*}, \ldots \in X^{*}$ e $y_{1}, y_{2}, \ldots \in Y$ com $\sum_{i=1}^{\infty}\left\|x_{i}^{*}\right\|\left\|y_{i}\right\|<\infty$ tais que $S=\sum_{i=1}^{\infty} x_{i}^{*}\left(y_{i}\right)$. 
Observação 85. Sejam $X$ e $Y$ espaços de Banach e $S=\sum_{i=1}^{\infty} x_{i}^{*}\left(y_{i}\right)$ um operador nuclear. A série $\sum_{i=1}^{\infty} x_{i}^{*}\left(y_{i}\right)$ converge em $L(X, Y)$. Portanto, todo operador nuclear $S: X \rightarrow Y$ é aproximável. Com efeito, $\lim _{n \rightarrow \infty}\left\|S^{\prime}-S_{n}\right\|=0$, onde $S_{n}=\sum_{i=1}^{n} x_{i}^{*}\left(y_{i}\right)$.

Observação 86. Segue da Proposição 50, p. 12, e da Proposição 51, p. 12, que a classe $K$ de todos os operadores compactos e a classe SS de todos os operadores estritamente singulares, respectivamente, são ideais de operadores.

É possível, por meio de regras específicas, associar a um ideal de operadores $\mathcal{U}$ um novo ideal de operadores. Tal associação, que representaremos por nova $: \mathcal{U} \rightarrow \mathcal{U}^{\text {nova }}$, definindo um ideal de operadores $\mathcal{U}^{\text {nova }}$ para todo ideal de operadores $\mathcal{U}$ é dita um procedimento.

Algumas propriedades especiais dos procedimentos:

(P1) (monotonicidade) Sejam $\mathcal{U}_{1}$ e $\mathcal{U}_{2}$ ideais de operadores. Se $\mathcal{U}_{1} \subseteq \mathcal{U}_{2}$ então $\mathcal{U}_{1}^{\text {nova }} \subseteq$ $\mathcal{U}_{2}^{\text {nova }}$;

(P2) (idempotência) Para qualquer ideal de operadores $\mathcal{U}$ temos que $\left(\mathcal{U}^{\text {nova }}\right)^{\text {nova }}=\mathcal{U}^{\text {nova }}$.

Um procedimento monótono e idempotente é dito um procedimento envoltório se $\mathcal{U} \subseteq$ $\mathcal{U}^{\text {nova }}$ e é dito um procedimento de núcleo se $\mathcal{U} \supseteq \mathcal{U}^{\text {nova }}$ para todo ideal de operadores $\mathcal{U}$.

Vejamos dois exemplos de procedimentos.

Definição 87. Seja $\mathcal{U}$ um ideal de operadores e sejam $X$ e $Y$ espaços de Banach. Um operador $S \in L(X, Y)$ pertence ao fecho $\mathcal{U}^{\text {Clos }}(X, Y)$ se existem operadores $S_{1}, S_{2}, S_{3}, \ldots \in$ $\mathcal{U}(X, Y)$ com $\lim _{n \rightarrow \infty}\left\|S-S_{n}\right\|=0$ em $L(X, Y)$.

Proposição 88. $\mathcal{U}^{\text {clos }}$ é um ideal de operadores, para qualquer ideal de operadores $\mathcal{U}$.

Prova. Obviamente, $I_{E} \in \mathcal{U}^{C l o s}$, onde $E$ denota o espaço de Banach de dimensão 1.

Sejam $S_{1}, S_{2} \in \mathcal{U}^{\text {Clos }}(X, Y)$, onde $X$ e $Y$ são espaços de Banach. Então existem operadores $S_{1}^{1}, S_{1}^{2}, \ldots, S_{1}^{n}, \ldots, S_{2}^{1}, S_{2}^{2}, \ldots, S_{2}^{n}, \ldots \in \mathcal{U}(X, Y)$ tais que dado $\varepsilon>0$ existem $n_{0}, n_{1} \in \mathbb{N}$ tais que $\left\|S_{1}-S_{1}^{n}\right\|<\frac{\varepsilon}{2}, \forall n \geq n_{o}$ e $\left\|S_{2}-S_{2}^{n}\right\|<\frac{\varepsilon}{2}, \forall n \geq n_{1}$. Temos que $S_{1}^{i}+S_{2}^{j} \in \mathcal{U}(X, Y), \forall i, j \in \mathbb{N}$. Consideremos a seqüência $\left\{S_{1}^{i}+S_{2}^{i}\right\}$ em $\mathcal{U}(X, Y)$. Seja $n_{3}=\max \left\{n_{\omega}, n_{1}\right\} \in \mathbb{N}$. Então

$$
\left\|S_{1}+S_{2}-\left(S_{1}^{i}-S_{2}^{i}\right)\right\| \leq\left\|S_{1}-S_{1}^{i}\right\|+\left\|S_{2}-S_{2}^{i}\right\|<\frac{\varepsilon}{2}+\frac{\varepsilon}{2}=\varepsilon, \forall n \geq n_{3} .
$$

Logo, temos que $S_{1}+S_{2} \in \mathcal{U}^{\text {Clos }}(X, Y)$. 
Sejam $X_{0}$ e $Y_{o}$ espaços de Banach, $T \in L\left(X_{0}, X\right), S \in \mathcal{U}^{\text {Clos }}(X, Y)$ e $R \in L\left(Y, Y_{0}\right)$. Então existe uma seqüencia de operadores $\left\{S_{i}\right\}$ em $\mathcal{U}(X, Y)$ tais que dado $\varepsilon>0$ existe $n_{o} \in \mathbb{N}$ tal que $\left\|S-S_{i}\right\|<\frac{\varepsilon}{\|R\|\|T\|}, \forall i \geq n_{o}$. Consideremos a seqüência de operadores $\left\{R S_{i}^{\prime} T\right\}$ em $\mathcal{U}\left(X_{o}, Y_{o}\right)$. Temos que

$$
\left\|R S T-R S_{i} T\right\| \leq\|R\|\left\|S-S_{i}\right\|\|T\|<\varepsilon, \forall n \geq n_{i} .
$$

Portanto, $\mathcal{U}^{\text {Clos }}$ é um ideal de operadores.

Proposição 89. A associação Clos $: \mathcal{U} \rightarrow \mathcal{U}^{\text {Clos }}$ é um procedimento envoltório, isto é,

$$
\mathcal{U} \subseteq \mathcal{U}^{\text {Clos }}
$$

Prova. Segue imediatamente das propriedades básicas de limites de seqüências de operadores.

Um ideal de operadores $\mathcal{U}$ é dito um ideal fechado se $\mathcal{U}=\mathcal{U}^{\text {Clos }}$.

Definição 90. Sejam $\mathcal{U}$ um ideal de operadores e $X$ e $Y$ espaços de Banach. Um operador $S \in L(X, Y)$ pertence ao radical $\mathcal{U}^{\text {Rad }}(X, Y)$ se para todo operador $T \in L(Y, X)$ existem operadores $U \in L(X)$ e $V \in \mathcal{U}(X)$ tais que

$$
U\left(I_{X}-T S\right)=I_{X}-V .
$$

Proposição 91. Seja $\mathcal{U}$ um ideal de operadores. Então $\mathcal{U}^{\text {Rad }}$ é um ideal de operadores.

Prova. A condição 1 da definição de ideal de operadores é trivial. Mostremos a condição 2. Sejam $S_{1}, S_{2} \in \mathcal{U}^{\text {Rad }}(X, Y)$, onde $X$ e $Y$ são espaços de Banach. Então dado $T \in L(Y, X)$, existem $U_{1} \in L(X)$ e $V_{1} \in \mathcal{U}(X) \operatorname{com} U_{1}\left(I_{X}-T S_{1}\right)=I_{X}-V_{1}$. Também existem $U_{2} \in L(X)$ e $V_{2} \in \mathcal{U}(X)$ tais que $U_{2}\left(I_{X}-\left(U_{1} T\right) S_{2}\right)=I_{X}-V_{2}$. Então

$$
\begin{gathered}
U_{2} U_{1}\left[I_{X}-T\left(S_{1}+S_{2}\right)\right]=U_{2}\left[U_{1}-U_{1} T\left(S_{1}+S_{2}\right)\right]=U_{2}\left[U_{1}-U_{1} T S_{1}-U_{1} T S_{2}\right]= \\
\begin{array}{c}
U_{2}\left[U_{1}\left(I_{X}-T S_{1}\right)-U_{1} T S_{2}\right]=U_{2}\left[I_{X}-V_{1}-U_{1} T S_{2}\right]=U_{2}\left(I_{X}-U_{1} T S_{2}\right)-U_{2} V_{1}= \\
=I_{X}-\underbrace{\left(V_{2}+U_{2} V_{1}\right)}_{\in \mathcal{U}(X)} .
\end{array}
\end{gathered}
$$

Finalmente, verifiquemos a condição 3. Sejam $X_{0}$ e $Y_{o}$ espaços de Banach, $T \in$ $L\left(X_{o}, X\right), S \in \mathcal{U}^{\text {Rad }}(X, Y)$ e $R \in L\left(Y, Y_{o}\right)$. Dado $J \in L\left(Y_{o}, X_{o}\right)$ existem $U \in L(X)$ e $V \in \mathcal{U}(X) \operatorname{com} U\left(I_{X}-(T J R) S\right)=I_{X}-V$. Definamos os operadores $U_{o}=I_{X_{0}}+J R S U T$ e $V_{0}=J R S V T$. Temos que $V_{o} \in \mathcal{U}\left(X_{0}\right)$, pois $V \in \mathcal{U}(X)$. Além disso, 


$$
\begin{gathered}
U_{o}\left(I_{X_{\circ}}-J R S T\right)=\left(I_{X_{o}}+J R S U T\right)\left(I_{X_{o}}-J R S T\right)= \\
I_{X_{o}}-J R S T+J R S U T-J R S U T J R S T= \\
I_{X_{o}}-J R S T+J R S(U T-U T J R S T)= \\
I_{X_{o}}-J R S T+J R S U\left(I_{X}-T J R S\right) T= \\
I_{X_{o}}-J R S T+J R S\left(I_{X}-V\right) T=I_{X_{o}}-J R S V T=I_{X_{o}}-V_{o} .
\end{gathered}
$$

Portanto $R S T \in \mathcal{U}^{\text {Rad }}\left(X_{o}, Y_{o}\right)$.

Proposição 92. A associação $\operatorname{Rad}: \mathcal{U} \rightarrow \mathcal{U}^{\text {Rad }}$ é um procedimento envoltório, isto é, $\mathcal{U} \subseteq \mathcal{U}^{\text {Rad }}$

Prova. A propriedade P1 (monotonicidade) é trivial. Sejam $X$ e $Y$ espaços de Banach, $S \in \mathcal{U}(X, Y)$ e $J \in L(Y, X)$. Definamos $U=I_{X}$ e $V=J S$. Então $V \in \mathcal{U}(X)$ e $U\left(I_{X}-\right.$ $J S)=I_{X}-J S=I_{X}-V$. Conseqüentemente, $S \in \mathcal{U}^{\text {Rad }}(X, Y)$. Isto prova que $\mathcal{U} \subseteq \mathcal{U}^{\text {Rad }}$.

Seja $S \in L(X, Y)$ pertencente a $\left(\mathcal{U}^{\text {Rad }}\right)^{\text {Rad }}$. Dado $J \in L(Y, X)$ temos que existem operadores $U_{1} \in L(X)$ e $V_{1} \in \mathcal{U}^{\text {Rad }}$ tais que $U_{1}\left(I_{X}-J S\right)=I_{X}-V_{1}$. Em particular, podemos encontrar também $U_{2} \in L(X)$ e $V_{2} \in \mathcal{U}(X)$ com $U_{2}\left(I_{X}-V_{1}\right)=I_{X}-V_{2}$. Conseqüentemente,

$$
U_{2} U_{1}\left(I_{X}-J S\right)=U_{2}\left(I_{X}-V_{1}\right)=I_{X}-V_{2}
$$

implicando que $S \in \mathcal{U}^{\text {Rad }}(X, Y)$. Portanto, $\left(\mathcal{U}^{\text {Rad }}\right)^{\text {Rad }} \subseteq \mathcal{U}^{\text {Rad }}$. Como a inclusão contrária é imediata, segue que $\left(\mathcal{U}^{\text {Rad }}\right)^{\text {Rad }}=\mathcal{U}^{\text {Rad }}$, isto é, a associação Rad satisfaz P2 (idempotência).

Proposição 93. Para todo ideal de operadores $\mathcal{U}$ o radical $\mathcal{U}^{\text {Rad }}$ é um ideal fechado.

Prova. Seja $S \in L(X, Y)$ pertencente a $\left(\mathcal{U}^{\text {Rad }}\right)^{\text {Clos }}(X, Y)$. Dado $J \in L(Y, X)$ temos que existe $S_{o} \in \mathcal{U}^{\operatorname{Rad}}(X, Y)$ tal que $\|J\|\left\|S-S_{o}\right\| .<1$. Então existem operadores $U_{o} \in L(X)$ e $V_{o} \in \mathcal{U}(X)$ tais que

$$
U_{o}\left(I_{X}-\left[I_{X}-J\left(S-S_{o}\right)\right]^{-1} J S_{o}\right)=I_{X}-V_{o}
$$

onde $\left[I_{X}-J\left(S-S_{o}\right)\right]^{-1}$ existe pela Proposição 52 .

Definimos $U=U_{o}\left[I_{X}-J\left(S-S_{o}\right)\right]^{-1}$. Então segue de (2.16) que

$$
U\left[I_{X}-J S\right]=U_{o}\left[I_{X}-J\left(S-S_{o}\right)\right]^{-1}\left[I_{X}-J S\right]=
$$




$$
\begin{gathered}
=U_{o}\left[I_{X}-J\left(S-S_{0}\right)\right]^{-1}\left[I_{X}-J\left(S-S_{0}\right)-J S_{o}\right]= \\
=U_{o}\left(I_{X}-\left[I_{X}-J\left(S-S_{0}\right)\right]^{-1} J S_{0}\right)=I_{X}-V_{o} .
\end{gathered}
$$

Portanto, $S \in \mathcal{U}^{\text {Rad }}(X, Y)$. Isto prova que $\left(\mathcal{U}^{\text {Rad }}\right)^{\text {Clos }}(X, Y) \subseteq \mathcal{U}^{\text {Rad }}(X, Y)$ para quaisquer espaços de Banach $X$ e $Y$.

Proposição 94. Seja $\mathcal{U}$ um ideal de operadores. Então $\mathcal{U}^{\text {Rad }}=\left(\mathcal{U}^{\text {Clos }}\right)^{\text {Rad }}$.

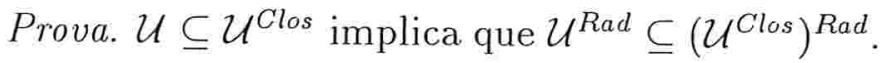

Considerando os ideais de operadores $K$ (dos operadores compactos) e $\mathcal{F}$ (dos operadores de posto finito) temos que $K=\mathcal{F}^{\text {Clos }}$. Segue da Proposição 94 que $K^{\text {Rad }}=$ $\left(\mathcal{F}^{\text {Clos }}\right)^{\text {Rad }}=\mathcal{F}^{\text {Rad }}$.

Definição 95. Os operadores pertencentes ao radical $\mathcal{F}^{\text {Rad }}=K^{\text {Rad }}$ são ditos Operadores Inessenciais ou Operadores de Gohberg; esta classe de operadores é denotada por In. Estudaremos os operadores inessenciais com mais detalhes no capítulo 5.

Proposição 96. Seja $S \in \mathcal{U}^{\text {Rad }}(X, Y)$, onde $X$ e $Y$ são espaços de Banach. Então para todo $J \in L(Y, X)$ existe $U \in L(X)$ e $V_{1}, \quad V_{2} \in \mathcal{U}(X)$ tais que $U\left(I_{X}-J S\right)=I_{X}-V_{1}$ e $\left(I_{X}-J S^{\prime}\right) U=I_{X}-V_{2}$.

Prova. Por hipótese existem operadores $U \in L(X)$ e $V_{1} \in \mathcal{U}(X)$ tais que $U\left(I_{X}-J S\right)=$ $I_{X}-V_{1}$. Definindo $R=I_{X}-U=I_{X}-I_{X}+V_{1}-U J S=V_{1}-U J S \in \mathcal{U}^{\text {Rad }}(X)$ temos que existem $U_{o} \in L(X)$ e $V_{o} \in \mathcal{U}(X)$ tais que $U_{o}\left(I_{X}-R\right)=I_{X}-V_{o}$. Daí, $U_{o}-U_{o} R=I_{X}-V_{o}$, implicando que $U_{o}-U_{o}\left(I_{X}-U\right)=I_{X}-V_{o}$, que por sua vez implica que $U_{o}-U_{o}+U_{o} U=$ $I_{X}-V_{o}$. Então $U_{o} U+V_{o}=I_{X}$, implicando que

$$
\begin{gathered}
\left(I_{X}-J S\right) U=I_{X}\left(I_{X}-J S\right) U=U_{o} U\left(I_{X}-J S\right) U+V_{o}\left(I_{X}-J S\right) U= \\
=U_{o}\left(I_{X}-V_{1}\right) U+V_{o}\left(I_{X}-J S\right) U= \\
=U_{o} U-U_{o} V_{1} U+V_{o} U-V_{o} J S U+V_{o}-V_{o}=I_{X}-V_{2},
\end{gathered}
$$

onde $V_{2}=U_{0} V_{1} U+V_{o}\left(I_{X}-U\right)+V_{o} J S U$.

Proposição 97. Sejam $X$ e $Y$ espaços de Banach $e S \in \mathcal{U}^{\text {Rad }}(X, Y)$. Então para qualquer operador $J \in L(X, Y)$ existem operadores $U \in L(Y), V_{1}, V_{2} \in \mathcal{U}(Y)$ tais que $U\left(I_{Y}\right.$ $S J)=I_{Y}-V_{1} e\left(I_{Y}-S J\right) V=I_{Y}-V_{2}$.

Prova. Temos que $S J \in \mathcal{U}^{\text {Rad }}(Y)$, pois $S \in \mathcal{U}^{\text {Rad }}(X, Y)$. Aplicando a Proposição 96 ao operador $S J$ obtemos o resultado desejado. 


\subsection{Teoria de perturbação de operadores de Fredholm}

O objetivo dessa seção é apresentar um resultado importante de perturbação de operadores de Fredholm por operadores estritamente singulares que será utiliza.da no Capítulo 4 (veja Teorema 149). Introduziremos também o conceito de $\Phi$-ideais e apresentaremos um resultado de perturbação de operadores de Fredholm por operadores em um $\Phi$-ideal que utilizaremos no Capítulo 6 (veja Teorema 203).

Consideremos uma álgebra $\mathcal{A}(X)$ de operadores lineares sobre um espaço de Banach $X$. $\mathrm{O}$ conjunto dos operadores de Fredholm em $\mathcal{A}(X)$ será denotado por $\Phi(\mathcal{A}(X))$. Também é utilizada a notação $\Phi(X)$ para o conjunto $\Phi(L(X))$. Seja $\mathcal{F}(\mathcal{A}(X))$ o ideal (bilateral) dos operadores de posto finito em $\mathcal{A}(X)$; para $\mathcal{F}(L(X))$ escrevemos apenas $\mathcal{F}(X)$.

Definição 98. Seja $X$ um espaço de Banach. Um ideal bilateral $\mathcal{J}$ em $L(X)$ é dito um $\Phi-$ ideal se

(a) $\mathcal{J} \supset \mathcal{F}(X)$;

(b) $I_{X}-K \in \Phi(X), \forall K \in \mathcal{J}$.

Em particular, o ideal (bilateral) In de todos os operadores inessenciais é um $\Phi$-ideal. Também é um $\Phi$-ideal a classe $\mathcal{F}$ de todos os operadores de posto finito.

Lema 99. Sejam $X$ e $Y$ espaços de Banach e $T \in L(X, Y)$. Seja $S \in L(X, Y)$ tal que $\|S\|<\gamma(T)$, onde $\gamma(T)$ é o módulo mínimo de T. Então

(i) $\alpha(T+S) \leq \alpha(T)$;

(ii) $\operatorname{dim}(Y / \overline{R(T+S)}) \leq \operatorname{dim}(Y / \overline{R(T)})$, isto é, $\bar{\beta}(T+S) \leq \bar{\beta}(T)$.

Prova. (i) Seja $x \neq 0$ em $N(T+S)$. Então

$$
\gamma(T)\|[x]\| \leq\|T(x)\|=\|S(x)\| \leq\|S\|\|x\|<\gamma(T)\|x\|,
$$

onde $[x] \in X / N(T)$. Deste modo, $\|x\|>\|[x]\|=d(x, N(T))$, implicando, pela. Proposição 56, que $\alpha(T+S) \leq \alpha(T)$.

(ii) Segue da. Proposição 28 e da. Proposição 74 que $\left\|S^{*}\right\|=\|S\|<\gamma(T)=\gamma\left(T^{*}\right)$. Pela. Proposição 77 e pelo que provamos em (i) temos que

$$
\bar{\beta}(T+S)=\alpha\left(T^{*}+S^{*}\right) \leq \alpha\left(T^{*}\right)=\bar{\beta}(T) .
$$


Corolário 100. Sejam $X$ e $Y$ espaços de Banach e $T \in L(X, Y)$ um operador que possui inversa contínua. Se $S \in L(X, Y)$ é tal que $\|S\|<\gamma(T)=1 /\left\|T^{-1}\right\|$ então $\bar{\beta}(T)=$ $\bar{\beta}(T+S)$.

Prova. Para $x \in X$ escolhamos um número inteiro positivo $n$ tal que $\frac{\|S\|}{n}<\gamma(T)-\|S\|$. Para $0 \leq k \leq n$ temos que

$$
\left\|\left(T+S-\frac{k}{n} S\right)(x)\right\| \geq\|T(x)\|-\left\|\left(1-\frac{k}{n}\right) S(x)\right\| \geq(\gamma(T)-\|S\|)\|x\| .
$$

Deste modo, $T+S$ possui inversa contínuá e

$$
\gamma(T+S-(k / n) S) \geq \gamma(T)-\|S\|
$$

para $0 \leq k \leq n$. Então, pelo Lema 99 , segue que

$$
\bar{\beta}\left(T+S-\frac{k+1}{n} S\right)=\bar{\beta}\left(T+S-\frac{k}{n} S-\frac{1}{n} S\right) \leq \bar{\beta}\left(T+S-\frac{k}{n} S\right) .
$$

Segue de (2.18) e do Lema 99 que

$$
\bar{\beta}(T) \leq \bar{\beta}(T+S) \leq \bar{\beta}(T) .
$$

Portanto, $\bar{\beta}(T)=\bar{\beta}(T+S)$.

Lema 101. Sejam $X$ e $Y$ espaços de Banach e $M$ um subespaço de $X$. Se $T \in \Phi_{+}(X, Y) \cup$ $\Phi_{-}(X, Y), T_{\left.\right|_{M}}$ é a restrição de $T$ a $M$ e $\operatorname{dim}(X / M)=n<\infty$ então $i(T)=i\left(T_{\left.\right|_{M}}\right)+n$.

Prova. É suficiente fazer a prova para o caso em que $n=1$. Suponhamos que $X=$ $M \oplus[x]$, onde $[x]$ denota o subespaço de $X$ gerado por algum $x \in X$. Então temos que $R(T)=R\left(T_{\left.\right|_{M}}\right) \oplus V$, onde $V$ é o subespaço de $Y$ gerado por $T(x)$ quando $T(x) \notin R\left(T_{\left.\right|_{M}}\right)$ ou $V=\{0\}$ quando $T(x) \in R\left(T_{\left.\right|_{M}}\right)$.

Se $T(x) \notin R\left(T_{\left.\right|_{M}}\right)$ então é fácil verificar que $\beta\left(T_{\left.\right|_{M}}\right)=\beta(T)+1$ e que $N\left(T_{\left.\right|_{M}}\right)=N(T)$. Portanto, temos que $i(T)=i\left(T_{\left.\right|_{M}}\right)+1$.

Se $T(x) \in R\left(T_{\left.\right|_{M}}\right)$ então $R(T)=R\left(T_{\left.\right|_{M}}\right)$ e existe um $m \in M$ tal que $T_{\left.\right|_{M}}(m)=T(x)$. Isto implica que $N(T)=N\left(T_{\left.\right|_{M}}\right) \oplus[x-m]$, onde $[x-m]$ é o subespaço de $N(T)$ gerado por $x-m$. Deste modo temos que $\alpha(T)=\alpha\left(T_{\left.\right|_{M}}\right)+1$ e $i(T)=i\left(T_{\left.\right|_{M}}\right)+1$.

Proposição 102. Sejam $X$ e $Y$ espaços de Banach e $T \in \Phi_{+}(X, Y) \cup \Phi_{-}(X, Y)$. Existe um número $\gamma(T)>0$ tal que se $S \in L(X, Y)$ satisfaz $\|S\|<\gamma(T)$ então 
(i) $R(T+S)$ é fechada, $\alpha(T+S) \leq \alpha(T)$ e $\beta(T+S) \leq \beta(T)$; (ii) $i(T+S)=i(T)$.

Em particular, o conjunto de todos os operadores em $\Phi_{+}(X, Y) \cup \Phi_{-}(X, Y)$ com índice igual a um valor fixado é aberto.

Prova. (i) Mostraremos primeiramente que $T+S$ possui imagem fechada. Suponhamos que $\alpha(T)<\infty$. Se $R(T+S)$ não é fechada então segue, pela Proposição 49, que existe um subespaço fechado de dimensão infinita $M$ de $X$ tal que para qualquer $x \in M$ temos $\|(T+S)(x)\|<(\gamma(T)-\|S\|)\|x\|$. Então para cada $x \neq 0$ em $M$ temos que

$$
\gamma(T) d(x, N(T)) \leq\|T(x)\| \leq\|S(x)\|+\|(T+S)(x)\|<(\|S\|+\gamma(T)-\|S\|)\|x\| .
$$

Portanto temos que $d(x, N(T))<\|x\|$, para qualquer $x \neq 0$ em $M$. Então, pela Proposição 56, temos que $\operatorname{dim}(M) \leq \operatorname{dim}(N(T))=\alpha(T)<\infty$, que é uma contradição. Deste modo, $R(T+S)$ é fechada.

Suponhamos que $\beta(T)<\infty$. Segue da Proposição 28 e da Proposição 74 que $\left\|S^{*}\right\|=$ $\|S\|<\gamma(T)=\gamma\left(T^{*}\right)$. Além disso, pela Proposição 77, temos que $\alpha\left(T^{*}\right)=\beta(T)<\infty$. Então pelo que temos provado, concluímos que $R\left(T^{*}+S^{*}\right)$ é fechada e

$$
\beta(T)=\alpha\left(T^{*}\right) \geq \alpha\left(T^{*}+S^{*}\right)
$$

Como $T^{*}+S^{*}=(T+S)^{*}$ então obtemos de (2.19), pela Proposição 77, que

$$
\beta(T) \geq \alpha\left(T^{*}+S^{*}\right)=\dot{\alpha}\left((T+S)^{*}\right)=\beta(T+S)
$$

e que $R(T+S)$ é fechada.

Finalmente, segue da. Proposição 99 que $\alpha(T) \geq \alpha(T+S)$.

(ii) Definimos a aplicação $\varphi:[0,1] \rightarrow \mathbb{Z} \cup\{-\infty,+\infty\}$ por $\varphi(\lambda)=i(T+\lambda S), \forall \lambda \in[0,1]$. Consideremos em $[0,1]$ a topologia usual e em $\mathbb{Z}$ a topologia discreta, isto é, pontos são abertos. Mostraremos que $\varphi$ é contínua, pois isto implica que $\varphi([0,1])$ é conexo e portanto consiste de um único ponto, implicando que $i(T)=\varphi(0)=\varphi(1)=i(T+S)$.

Primeiramente provaremos a continuidade de $\varphi$ para $\|S\|$ suficientemente pequeno. Suponhamos que $\alpha(T)<\infty$. Então existe um subespaço fechado $M$ de $X$ tal que $X=$ $M \oplus N(T)$. Seja $T_{\left.\right|_{M}}$ a restrição de $T$ a $M$. Então $T_{\left.\right|_{M}}$ possui uma inversa contínua desde que é contínuo, injetor e $R\left(T_{\left.\right|_{M}}\right)=R(T)$. Então se $\|S\|<\gamma\left(T_{\left.\right|_{M}}\right)$ segue, por (i) e pelo 
Corolário 100, que

$$
\alpha\left(T_{\left.\right|_{M}}+S\right)=\alpha\left(T_{\left.\right|_{M}}\right)=0 \quad \beta\left(T_{\left.\right|_{M}}+S\right)=\beta(T)
$$

Segue de (2.20) e do Lema 101 que para $\|S\|<\gamma\left(T_{\left.\right|_{M}}\right)$,

$$
i(T)=i\left(T_{\left.\right|_{M}}\right)+\alpha(T)=i\left(T_{\left.\right|_{M}}+S\right)+\alpha(T)=i(T+S)
$$

Suponhamos agora que $\beta(T)<\infty$. Aplicando (2.21) a. $T^{*}$ e $S^{*}$ obtemos que

$$
i(T+S)=-i\left(T^{*}+S^{*}\right)=-i\left(T^{*}\right)=i(T)
$$

para $\|S\|$ suficientemente pequeno.

Dado $\lambda_{o} \in[0,1]$, segue de (i) que $T+\lambda_{o} S \in \Phi_{+}(X, Y) \cup \Phi_{-}(X, Y)$ e possui imagem fechada. Deste modo, pelos resultados obtidos acima segue que

$$
\varphi(\lambda)=i\left(T+\lambda_{\circ} S+\left(\lambda-\lambda_{0}\right) S\right)=i\left(T+\lambda_{o} S\right)=\varphi\left(\lambda_{\circ}\right)
$$

para $\lambda$ suficientemente próximo de $\lambda_{o}$. Deste modo, $\varphi$ é contínua.

Corolário 103. Sejam $X$ e $Y$ espaços de Banach e $T \in \Phi_{+}(X, Y) \cup \Phi_{-}(X, Y)$. Suponhamos que $S \in L(X, Y)$ satisfaz $\|S\|<\gamma(T)$, onde $\gamma(T)$ é o módulo mínimo de T. Então existe um número $\rho>0$ tal que $\alpha(T+\lambda S)$ e $\beta(T+\lambda S)$ são constantes no anel $0<|\lambda|<\rho$.

Prova. Suponhamos primeiramente que $\alpha(T)<\infty$. Para $x \in N(T+\lambda S)$ e $\lambda \neq 0$ temos que $T(x)=-\lambda S(x)$ e então

$$
S(x) \in R(T)=R_{1} \text { e } x \in S^{-1}\left(R_{1}\right)=D_{1} .
$$

Deste modo,

$$
-\lambda S(x)=T(x) \in T\left(D_{1}\right)=R_{2} \text { e } x \in S^{-1}\left(R_{2}\right)=D_{2} .
$$

Segue que

$$
N(T+\lambda S) \subset \cap_{k=1}^{\infty} D_{k}
$$

onde $D_{k}=S^{-1} R_{k}, R_{1}=R(T)$ e $R_{k+1}=T\left(D_{k}\right)$. É fácil ver que

$$
R_{1} \supset R_{2} \supset \cdots \text { e } D_{1} \supset D_{2} \supset \cdots
$$

Mostraremos por indução que $R_{n}$ e $D_{n}$ são subespaços fechados de $Y$ e de $X$, respectivamente. Temos por hipótese que $R_{1}$ é fechado e como $S$ é contínuo então $D_{1}$ também é fechado. Suponhamos que $R_{k}$ e $D_{k}$ são fechados. Pelo Lema 79 temos que $R_{k+1}=T\left(D_{k}\right)$ 
é fechado e portanto $D_{k+1}=S^{-1} R_{k+1}$ é fechado pois $S$ é contínuo. Então $D_{n}$ e $R_{n}$ são fechados por indução. Sejam

$$
X_{1}=\cap_{k=1}^{\infty} D_{k} \text { e } Y_{1}=\cap_{k=1}^{\infty} R_{k}
$$

Segue da definição de $D_{k}$ e $R_{k}$ que $T\left(X_{1}\right) \subset Y_{1}$ e $S\left(X_{1}\right) \subset Y_{1}$. Sejam $T_{1}$ e $S_{1}$ os operadores $T$ e $S$, respectivamente, restritos a $X_{1}$ com imagens em $Y_{1}$. Como $T$ é contínuo e $X_{1}$ é fechado então $T_{1}$ é um operador fechado. Mostraremos que $R\left(T_{1}\right)=Y_{1}$. Seja $y$ um elemento em $Y_{1}=\cap_{n=1}^{\infty} T\left(D_{n}\right)$. Então para cada $n \geq 1$ existe um $x_{n} \in D_{n}$ tal que $T\left(x_{n}\right)=y$. Como $N(T)$ é de dimensão finita e $D_{n} \supset D_{n+1}$ então existe $k_{o} \in \mathbb{Z}$ tal que

$$
N(T) \cap D_{k_{o}}=N(T) \cap D_{k}, \forall k>k_{o} .
$$

Então pelo modo como a seqüência $\left\{x_{k}\right\}$ foi escolhida, e como $D_{k} \subset D_{k_{o}}$, segue que

$$
x_{k}-x_{k_{o}} \in N(T) \cap D_{k_{o}}=N(T) \cap D_{k} \subset D_{k}, \forall k \geq k_{o} .
$$

Então $x_{k_{\circ}} \in \cap_{k \geq k_{o}} D_{k}=X_{1}$ e $T\left(x_{k_{o}}\right)=y$, implicando que $T_{1}$ é sobrejetora. Deste modo existe, pela Proposição 102, um número $\rho>0$ tal que para $|\lambda|<\rho$,

$$
\begin{gathered}
i(T+\lambda S)=i(T), \\
\beta\left(T_{1}+\lambda S\right)=\beta\left(T_{1}\right)=0 \text { e } \alpha\left(T_{1}+\lambda S_{1}\right)=i\left(T_{1}\right)=\alpha\left(T_{1}\right) .
\end{gathered}
$$

Segue de 2.22 que $N(T+\lambda S)=N\left(T_{1}+\lambda S_{1}\right)$, para $\lambda \neq 0$. Em particular,

$$
\alpha(T+\lambda S)=\alpha\left(T_{1}+\lambda S_{1}\right), \forall \lambda \neq 0
$$

Segue de (2.23), (2.24) e (2.25) que $\alpha\left(T+\lambda S^{\prime}\right)$ e $\beta(T+\lambda S)$ são constantes no anel $0<|\lambda|<\rho$, quando $\alpha(T)<\infty$. Se $\alpha(T)=\infty$ então $\beta(T)<\infty$ por hipótese. Assim como na prova da Proposição 102 aplicamos o resultado provado acima aos operadores conjugados apropriados para provar esse corolário no caso em que $\alpha(T)=\infty$.

Proposição 104. Sejam $X$ e $Y$ espaços de Banach e $T: X \rightarrow Y$ um operador com imagem fechada para o qual $\alpha(T)<\infty$. Seja $S: X \rightarrow Y$ um operador estritamente singular. Então $\alpha(T+S)<\infty, T+S$ possui imagem fechada e $i(T+S)=i(T)$.

Prova. Seja $X_{1} \subset X$ tal que $X=X_{1} \oplus N(T)$. Então $T_{\left.\right|_{X_{1}}}$ é um isomorfismo. Se $\operatorname{dim}(N(T+$ $\left.\left.S^{\prime}\right)\right)=\infty$ então existe um subespaço de dimensão infinita $Z$ de $X_{1}$ sobre o qual $T+S=0$, isto é, sobre o qual $S=-T$ é inversível. E isto contradiz a afirmação de que $S$ é estritamente singular. Supondo que $(T+S)(X)$ não é um subespaço fechado temos, pela. 
Proposição 49, que existe um subespaço de dimensão infinita $Z$ de $X_{1}$ tal que $\left\|(T+S)_{\mid Z}\right\|<$ $\left\|T_{\left.\right|_{X_{1}}}^{-1}\right\|^{-1}$. Então $S_{\left.\right|_{Z}}$ é um isomorfismo-e chegamos novamente a uma contradição.

Pelo que temos provado o número $i(T+t S)$ está definido para todo $t \in[0,1]$. Pela Proposição 102 temos que $i(T+t S)$ é uma função contínua de $t$ e, deste modo, uma constante, isto é, $i(T)=i(T+S)$.

Corolário 105. Seja $X$ um espaços de Banach. Para $S \in K(X)$ o operador $I_{X}-S$ é um operador de Fredholm com $i\left(I_{X}-S\right)=0$.

Prova. É uma conseqüência imediata da Proposição 104.

Proposição 106. Sejam $X$ e $Y$ espaços de Banach. Então $T \in \Phi_{-}(X, Y)$ se, e somente se, $\beta(T-S)<\infty$, para qualquer $S \in K(X, Y)$.

Prova. Se $T \in \Phi_{-}(X, Y)$ então $\beta(T-S)<\infty, \forall S \in K(X, Y)$, pela Proposição 104 . Suponhamos agora que $T \notin \Phi_{-}(X, Y)$. Se $\beta(T)=\infty$ então existe um $S \in K(X, Y)$ (a saber, $K=0)$ tal que $\beta(T-S)=\infty$, finalizando então a prova para esse caso. Se $\beta(T)<$ $\infty$ então $R(T)$ não é fechada. Seja $\left\{a_{n}\right\}$ uma seqüência em $\mathbb{Z}$ definida indutivamente por

$$
a_{1}=2, \quad a_{n}=2\left(1+\sum_{k=1}^{n-1} a_{k}\right), \quad n=2,3, \ldots
$$

Suponhamos que existem duas seqüências $\left\{y_{k}\right\} \subset Y$ e $\left\{y_{k}^{*}\right\} \subset Y^{*}$ tais que

$$
\left\|y_{k}\right\| \leq a_{k}, \quad\left\|y_{k}^{*}\right\|=1, \quad\left\|T^{*}\left(y_{k}^{*}\right)\right\|<1 / 2^{k} a_{k}, \quad y_{j}^{*}\left(y_{k}\right)=\delta_{j k}, j, k=1,2, \ldots
$$

Assumindo isto por um momento definimos o operador de posto finito

$$
S_{n}(x)=\sum_{k=1}^{n} T^{*}\left(y_{k}^{*}(x)\right) y_{k}, \quad n=1,2, \ldots
$$

Então para $n>m$ temos que

$$
\left\|S_{n}(x)-S_{m}(x)\right\| \leq \sum_{k=m+1}^{n} \| T^{*}\left(y_{k}^{*}\|\| x\|\| y_{k}\|\leq\| x \| \sum_{k=m+1}^{n} 2^{-k} \leq \frac{\|x\|}{2^{m}} \rightarrow 0\right.
$$

quando $m \rightarrow \infty$. Deste modo, $S_{n}$ converge em $L(X, Y)$ para o compacto

$$
S(x)=\sum_{k=1}^{\infty} T^{*}\left(y_{k}^{*}(x)\right) y_{k} .
$$

Segue de (2.27) que para cada $x \in X$ e cada $k \in \mathbb{N}$ temos 


$$
y_{k}^{*}(S(x))=T^{*}\left(y_{k}^{*}(x)=y_{k}^{*}(T(x)) .\right.
$$

Conseqüentemente, cada um dos $y_{k}^{*}$ anula $R(T-S)$. Como os $y_{k}^{*}$ são linearmente independentes então segue que $\beta(T-S)=\infty$ e isso prova o resultado.

Basta portanto encontrar seqüências satisfazendo (2.27). Faremos isso por indução. Como $R(T)$ não é fechado, então também não o é $R\left(T^{*}\right)$. Então existe $y_{1}^{*}$ tal que $\left\|y_{1}\right\|=1$ e $\left\|T^{*}\left(y_{1}^{*}\right)\right\|<1 / 4$ e existe um $y_{1}$ tal que $\left\|y_{1}\right\|<2$ com $y_{1}^{*}\left(y_{1}\right)=1$. Suponhamos que tenhamos encontrado $y_{1}, \ldots, y_{n-1}, y_{1}^{*}, \ldots, y_{n-1}^{*}$ satisfazendo (2.26). Então existe um $y_{n}^{*}$ que anula $y_{1}, \ldots, y_{n-1}$ tal que $\left\|y_{n}^{*}\right\|=1$ e $\left\|T^{*}\left(y_{n}^{*}\right)\right\|<1 / 2^{n} a_{n}$. Existe também um $y \in Y$ tal que $y_{n}^{*}(y)=1$ e $\|y\|<2$. Definimos

$$
y_{n}=y-\sum_{k=1}^{n-1} y_{k}^{*}(y) y_{k} .
$$

Então, por (2.26) e pela hipótese de indução temos que

$$
\left\|y_{n}\right\| \leq\|y\|\left(1+\sum_{k=1}^{n-1}\left\|y_{k}\right\|\right) \leq 2\left(1+\sum_{k=1}^{n-1} a_{k}\right)=a_{n} .
$$

Além disso, pelo modo como $y_{n}^{*}$ e $y_{n}$ foram escolhidos temos que $y_{n}^{*}\left(y_{n}\right)=1, y_{n}^{*}\left(y_{k}\right)=0$, para $1 \leq k<n$. Também temos que

$$
y_{k}^{*}\left(y_{n}\right)=y_{k}^{*}(y)-y_{k}^{*}(y)=0, \quad 1 \leq k<n .
$$

Deste modo, (2.27) ocorre para qualquer $n$.

Proposição 107. Sejam $X$ e $Y$ espaços de Banach e sejam $B, T \in L(X, Y)$, com $D(T) \subset$ $D(B)$. Defina $U$ como o conjunto dos $\lambda \in \mathbb{C}$ para os quais o operador $T+\lambda B$ é fechado, possui imagem fechada e um indice definido. Então

(i) U é aberto;

(ii) Se $C$ é uma componente conexa de $U$ (isto é, para algum $x \in U$, o conjunto $C$ é o maior subconjunto conexo de $U$ contendo $x$ ) então sobre $C$, com possíveis exceções de pontos isolados, temos que $\alpha(T+\lambda B)$ e $\beta(T+\lambda B)$ possuem valores constantes $n_{1}$ e $n_{2}$, respectivamente. Nos pontos isolados, $\alpha(T+\lambda B)>n_{1} \in \beta(T+\lambda B)>n_{2}$.

Prova. (i) Seja $\lambda \in U$. Então $T+\lambda B$ é um operador fechado, a imagem $R(T+\lambda B)$ é um subespaço fechado e $T+\lambda B$ possui um índice. Consideremos a bola aberta $B(\lambda, \gamma(T+\lambda B))$ de centro $\lambda$ e raio $\gamma(T+\lambda B)$, onde $\gamma(T+\lambda B)$ é o módulo mínimo do operador $T+\lambda B$. Seja $\mu \in B(\lambda, \gamma(T+\lambda B))$. Então $|\lambda-\mu|<\gamma(T+\lambda B)$. Observemos que 


$$
T+\mu B=(T+\lambda B)+(\mu-\lambda) B .
$$

Então pela. Proposição $102, T+\mu B$ é um operador fechado, a imagem $R(T+\mu B)$ é um subespaço fechado e $T+\mu B$ possui um índice, isto é, $\mu \in U$. Logo,

$$
B(\lambda, \gamma(T+\lambda B)) \subset U, \forall \lambda \in U \text {. }
$$

Portanto $U$ é um conjunto aberto.

(ii) Seja $C$ uma componente conexa de $U$. Como qualquer componente conexa de um conjunto aberto num espaço de escalares é também um conjunto aberto, segue que $C$ é um conjunto aberto. Seja $\alpha\left(\lambda_{o}\right)=n_{1}$ o menor inteiro que é atingido por $\alpha(\lambda)=\alpha(T+\lambda B)$ sobre $C$. Suponhamos que $\alpha\left(\lambda^{\prime}\right) \neq n_{1}$, para algum $\lambda^{\prime} \in C$.

Como $C$ é conexo então também é conexo por caminhos, implicando que existe um caminho $f:[0,1] \rightarrow C \operatorname{com} f(0)=\lambda_{o}$ e $f(1)=\lambda^{\prime}$ que determina um arco $\Gamma$ em $C$ unindo $\lambda_{o}$ e $\lambda^{\prime}$.

Como $C$ é um conjunto aberto segue pelo Corolário 103 que para cada $\mu \in \Gamma$ existe uma bola aberta $S(\mu) \subset C$ tal que $\alpha(\lambda)$ é constante sobre o conjunto $S(\mu)-\{\mu\}$.

Sendo $\Gamma$ compacto e conexo (imagem do compacto e conexo $[0,1]$ pela função contínua $f)$, segue que existe uma quantidade finita de pontos $\lambda_{1}, \lambda_{2}, \cdots, \lambda_{n}=\lambda^{\prime}$ sobre $\Gamma$ tais que as bolas abertas

$$
S\left(\lambda_{o}\right), \ldots, S\left(\lambda_{n}\right) \text { cobrem } \Gamma \text { e } S\left(\lambda_{i}\right) \cap S\left(\lambda_{i+1}\right) \neq \emptyset, \quad i=1, \ldots, n-1 .
$$

Afirmamos que $\alpha(\lambda)=\alpha\left(\lambda_{o}\right)=n_{1}, \forall \lambda \in S\left(\lambda_{o}\right)$. De fato, segue pela Proposição 102 que $\alpha(\lambda) \leq \alpha\left(\lambda_{o}\right)$ para $\lambda$ suficientemente próximo a $\lambda_{o}$. Vejamos

$$
\alpha(\lambda)=\alpha(T+\lambda B)=\alpha\left(\left(T+\lambda_{o} B\right)+\left(\lambda-\lambda_{0}\right) B\right) \leq \alpha\left(T+\lambda_{o} B\right)=\alpha\left(\lambda_{o}\right) .
$$

Como $\alpha\left(\lambda_{0}\right)$ é o valor mínimo de $\alpha(\lambda)$ sobre $C$ segue que $\alpha(\lambda)=\alpha\left(\lambda_{o}\right)$ para $\lambda$ suficientemente próximo de $\lambda_{0}$. Desde que $\alpha(\lambda)$ é constante para todo $\lambda \neq \lambda_{o}$ em $S\left(\lambda_{0}\right)$ e $S\left(\lambda_{i}\right) \cap S\left(\lambda_{i+1}\right) \neq \emptyset \quad(i=1, \cdots, n-1)$ então esta constante é $\alpha\left(\lambda_{o}\right)$. Portanto $\alpha(\lambda)$ é constante sobre o conjunto $S\left(\lambda_{i}\right)-\left\{\lambda_{i}\right\}$. Então segue por (2.28) e pelo fato de que $\alpha(\lambda)=\alpha\left(\lambda_{0}\right), \forall \lambda \in S\left(\lambda_{0}\right)$ que $\alpha(\lambda)=\alpha\left(\lambda_{0}\right)$ para todo $\lambda \neq \lambda^{\prime}$ em $S\left(\lambda^{\prime}\right)$ e $\alpha\left(\lambda^{\prime}\right)>n_{1}$.

Este resultado pode ser aplicado ao operador conjugado $T^{*}+\lambda B^{*}$ para provar o resultado para $\beta(T+\lambda B)=\alpha\left(T^{*}+\lambda B^{*}\right)$.

A seguir exibiremos resultados de perturbação de operadores de Fredholm por operadores de posto finito e por operadores em um $\Phi$-ideal. Porém, em preparação para 
apresentar tais resultados, apresentaremos algumas considerações e resultados envolvendo operadores de Riesz.

Sejam $X$ um espaço de Banach e $T: X \rightarrow X$ um operador linear. Denotaremos por $T^{n}: X \rightarrow X$ a composição $T \circ T \circ \cdots \circ T$ de $T$ por ele mesmo $n$ vezes, onde $n \in \mathbb{N}$. Definimos $T^{\circ}=I_{X}$ como sendo o operador identidade sobre $X$. Temos que

$$
N\left(T^{o}\right)=\{0\} \subset N(T) \subset N\left(T^{2}\right) \subset \ldots \subset N\left(T^{n}\right) \subset \ldots
$$

a qual chamaremos de cadeia de núcleos de $T$. Se existe um $n \in \mathbb{N}$ tal que $N\left(T^{n}\right)=$ $N\left(T^{n+1}\right)$ então $N\left(T^{n+1}\right)=N\left(T^{n+2}\right)$, pois se $x \in N\left(T^{n+2}\right)$ então $T^{n+1}(T(x))=0$, implicando que $T(x) \in N\left(T^{n+1}\right)=N\left(T^{n}\right)$ e isso implica que $x \in N\left(T^{n+1}\right)$. Isso implica que se existe um $n \in \mathbb{N}$ tal que $N\left(T^{n}\right)=N\left(T^{n+1}\right)$ então $N\left(T^{n}\right)=N\left(T^{n+m}\right)$, para $m=1,2,3, \ldots$ O menor número $n \in \mathbb{N}$ para o qual isso ocorre é chamado de comprimento da cadeia de núcleos de $T$ e o denotaremos por $p(T)$. Se $N\left(T^{n}\right) \neq N\left(T^{n+1}\right), \forall n \in \mathbb{N}$ então definimos $p(T)=\infty$.

Por outro lado temos que

$$
X \supset R(T) \supset R\left(T^{2}\right) \supset \ldots \supset R\left(T^{n}\right) \supset \ldots,
$$

a qual chamaremos de cadeia da imagem de $T$. Se existe um $n \in \mathbb{N}$ tal que $R\left(T^{n}\right)=$ $R\left(T^{n+1}\right)$ então $R\left(T^{n}\right)=R\left(T^{n+m}\right)$, para $m=1,2,3, \ldots$ O menor número $n \in \mathbb{N}$ para o qual isso ocorre é chamado de comprimento da cadeia da imagem de $T$ e o denotaremos por $q(T)$. Se $R\left(T^{n}\right) \neq R\left(T^{n+1}\right), \forall n \in \mathbb{N}$ então definimos $q(T)=\infty$.

Obviamente $p(T)=0$ significa que $T$ é injetor e $q(T)=0$ significa que $T$ é sobrejetor.

Proposição 108. Dado um operador linear $T=I_{X}-S: X \rightarrow X$, onde $X$ é um espaço de Banach e $S \in \mathcal{F}(X)$, então $p(T)=q(T)<\infty$.

Prova. Temos que

$$
T^{n}=\left(I_{X}-S^{n}\right)^{n}=I_{X}-\left[n S-\left(\begin{array}{l}
n \\
2
\end{array}\right) S^{2}+\cdots+(-1)^{n-1} S^{n}\right]=I_{X}-S_{n}, \forall n \geq 1
$$

onde $S_{n}=\left[n S-\left(\begin{array}{c}n \\ 2\end{array}\right) S^{2}+\cdots+(-1)^{n-1} S^{n}\right]$.

Segue, obviamente, que $N\left(T^{n}\right) \subset R\left(S_{n}\right) \subset R(S)$. Como $S \in \mathcal{F}(X)$ então $S_{n} \in \mathcal{F}(X)$, implicando, pelo Corolário 105, que $\alpha\left(I_{X}-S_{n}\right)=\beta\left(I_{X}-S_{n}\right)$. Deste modo, $\beta\left(T^{n}\right)=$ $\beta\left(I_{X}-S_{n}\right)=\alpha\left(I_{X}-S_{n}\right)=\alpha\left(T^{n}\right)$ é constante a partir de um mesmo $n \in \mathbb{N}$. Logo, $p(T)=q(T)<\infty$. 
Definição 109. Sejam $X$ um espaço de Banach e $T \in L(X)$. Um número $\lambda \in \mathbb{C}$ é dito um ponto de Riesz de $T$ se para-operador $\lambda I_{X}-T$ são válidas as seguintes propriedades:

(i) $\lambda I_{X}-T$ é aberto;

(ii) $R\left(\lambda I_{X}-T\right)$ é fechada;

(iii) $i\left(\lambda I_{X}-T\right)=0$;

(iv) $p\left(\lambda I_{X}-T\right)=q\left(\lambda I_{X}-T\right)<\infty$.

O conjunto $P_{T}$ de todos os pontos de Riesz é chamado região de Riesz de T e o número $r_{P}(T)=\inf \{r>0: \lambda$ é um ponto de Riesz de T para todo $|\lambda|>r\}$

é o raio de Riesz de $T$. Dizemos que $T$ é um operador de Riesz se $r_{P}(T)=0$, isto é, se todo $\lambda \neq 0$ é um ponto de Riesz de $T$.

Definição 110. Sejam $X$ um espaço de Banach e $T \in L(X)$. O conjunto $\Phi_{T}=\{\lambda \in \mathbb{C}$ : $\left.\lambda I_{X}-T \in \Phi(X)\right\}$ é dito região de Fredholm de T. Os pontos de $\Phi_{T}$ são ditos pontos de Fredholm de T.

Observação 111. Segue da Proposição 102 que $\Phi_{T}$ é um conjunto aberto e da Proposição 107, com $I_{X}$ no lugar de $B$, que sobre qualquer componente conexa de $\Phi_{T}$ a função indice $\lambda \rightarrow i\left(\lambda I_{X}-T\right)$ é constante.

Proposição 112. Sejam $X$ um espaço de Banach e $T \in L(X)$. Então T é um operador de Riesz em $X$ se, e somente se, para todo $\lambda \neq 0, \alpha\left(\lambda I_{X}-T\right)<\infty$ e $\beta\left(\lambda I_{X}-T\right)<\infty$.

Prova. Se $T$ é um operador de Riesz então trivialmente $\alpha\left(\lambda I_{X}-T\right)<\infty$ e $\beta\left(\lambda I_{X}-T\right)<$ $\infty, \forall \lambda \neq 0$.

Reciprocamente, se $\alpha\left(\lambda I_{X}-T\right)<\infty$ e $\beta\left(\lambda I_{X}-T\right)<\infty, \forall \lambda \neq 0$ então $\Phi_{T}$ possui somente uma componente conexa $C \supset \mathbb{C}-\{0\}$, implicando, pela Observação 111, que todo $\lambda \in C$ é um ponto de Riesz.

Proposição 113. Se $X$ é um espaço de Banach então todo operador linear $T: X \rightarrow X$ em um $\Phi$-ideal $\mathcal{J}$ sobre $X$ é um operador de Riesz.

Prova. Como $T \in \mathcal{J}$ então $\lambda I_{X}-T$ é um operador de Fredholm para todo $\lambda \in \mathbb{C}$. Então segue da Proposição 112 que $T$ é um operador de Riesz. 
Lema 114. Sejam $X, Y, Z$ e $W$ espaços de Banach. Se os operadores $S \in L(Z, X), T \in$ $\Phi(X, Y)$ e $K \in L(Y, W)$ são tais que $S T=C$ (ou $T K=D$ ) com $i(C)=0$ (respectivamente, $i(D)=0$ ) então $S \in \Phi(Z, X)$ (respectivamente, $K \in \Phi(Y, W)$ ) e $i(S)=-i(T)$ (respectivamente, $i(K)=-i(T))$.

Prova. O resultado é uma conseqüência imediata da Proposição 80(iii), p. 25.

Proposição 115. Sejam $X$ um espaço de Banach, $T \in \Phi(X)$ e $S: X \rightarrow X$ um operador de posto finito. Então $i(T+S)=i(T)$.

Prova. Segue da Observação 122 que $T+S$ possui um índice finito. Pela Proposição 120 existem operadores $B \in L(X)$ e $H \in \mathcal{F}(X)$ tais que $B T=I_{X}-H$. Então, pelo Corolário 105 temos que $i\left(I_{X}-H\right)=0$. Então pelo Lema 114 temos que $i(B)=-i(T)$. Além disso,

$$
B(T+S)=B T+B S=I_{X}-H+B S=I_{X}-H_{1},
$$

onde $H_{1}=H-B S \in \mathcal{F}(X)$. Então pela Proposição 80(iii) temos que

$$
i(T+S)=-i(B)=i(T)
$$

Proposição 116. Seja $X$ um espaço de Banach. Para todo $T \in \Phi(X)$ e para todo $K$ em um $\Phi$-ideal $\mathcal{J}$ em $L(X)$ temos que $i(T+K)=i(T)$.

Prova. Como $T \in \Phi(X)$ então segue pela Proposição 120 que existe um $B \in L(X)$ e $J \in \mathcal{F}(X)$ tais que $B T=I_{X}-J$. Pelo Corolário 105 temos que $i(I-J)=0$. Então pelo Lema 114 temos que $i(B)=-i(T)$. Além disso,

$$
B(T+K)=B T+B K=I_{X}-J+B K=I_{X}-J_{1}
$$

$\operatorname{com} J_{1}=J-B K \in \mathcal{J}$. Segue da Proposição 113 que $J_{1}$ é um operador de Riesz em $X$ e portanto $i\left(I_{X}-J_{1}\right)=0$ e $i(T+K)=-i(B)=i(T)$.

\subsection{Caracterizações de operadores de Fredholm}

Apresentaremos a seguir algumas caracterizações dos operadores de Fredholm. Tais caracterizações dependem dos conceitos de ideais de operadores e radical de um operador. 
Lema 117. Seja $G$ um subespaço de um espaço vetorial $X$ tal que $X=G+V$, onde $V$ é um subespaço de dimensão finita de $F$. Então $G$ é de codimensão finita em $F$.

Prova. Seja $\left\{v_{1}, \ldots, v_{n}\right\}$ uma base algébrica de $V$. Dado $x \in X$ temos que $x=g+\alpha_{1} v-$ $1+\cdots+\alpha_{n} v_{n}$, onde $\alpha_{1}, \ldots, \alpha_{n} \in \mathbb{C}$. Temos ainda que $[x]=x+G=\alpha_{1} v_{1}+\cdots+\alpha_{n} v_{n}+G$. Afirmamos que $\left\{\left[v_{1}\right], \ldots,\left[v_{n}\right]\right\}$ é uma base algébrica para $X / G$. De fato, os elementos $\left[v_{1}\right], \ldots,\left[v_{n}\right]$ são linearmente independentes pois se $\beta_{1}\left[v_{1}\right]+\cdots+\beta_{n}\left[v_{n}\right]=[0]$ então $\alpha_{1} v_{1}+$ $\cdots+\beta_{n} v_{n} \in G$, implicando que $\alpha_{1} v_{1}+\cdots+\beta_{n} v_{n}=0$ e portanto $\beta_{i}=0$, onde $i=1, \ldots n$. Além disso, dado $x \in X$ temos que $[x]=\left[\alpha_{1} v_{1}+\cdots+\alpha_{n} v_{n}\right]=\alpha_{1}\left[v_{1}\right]+\cdots+\alpha_{n}\left[v_{n}\right]$, onde $\alpha_{1}, \ldots, \alpha_{n} \in \mathbb{C}$.

Lema 118. Seja $X$ um espaço vetorial. Se $C_{1} \subset C_{2} \subset X$ e $C_{1}$ é de codimensão finita em $X$ então $C_{1}$ é de codimensão finita em $C_{2}$.

Prova. Seja $\left\{\left[v_{1}\right], \ldots,\left[v_{m}\right]\right\}$ uma base algébrica de $X / C_{1}$. Dado $[c] \in C_{2} / C_{1}$ temos que $[c]=c+C_{1}$, onde $c \in C_{2}$. Além disso, temos que $[c] \in X / C_{1}$ implicando que existem escalares $\alpha_{1}, \ldots, \alpha_{n} \in \mathbb{C}$ tais que $[c]=\alpha_{1}\left[v_{1}\right]+\cdots+\alpha_{n}\left[v_{m}\right]$. Se $\left[v_{1}\right], \ldots,\left[v_{m}\right]$ são linearmente dependentes então algum $\left[v_{i}\right]$, onde $i=1, \ldots, n$, é uma combinação linear dos restantes. Deste modo, obtemos uma base algébrica para $C_{2} / C_{1}$ que é composta por uma quantidade menor ou igual a $m$. Logo, $C_{1}$ é de codimensão finita em $C_{2}$.

Lema 119. Seja $K: X \rightarrow X$ um isomorfismo em um espaço vetorial normado $X e$ $B: X \rightarrow X$ um operador de posto finito. Então o operador linear $K+B$ tem imagem fechada.

Prova. Suponhamos que o operador $K+B$ não possua imagem fechada. Então pela Proposição 49 existe um subespaço de dimensão infinita $Z$ de $X$ tal que a restrição $(K+$ $B)_{\left.\right|_{Z}}$ de $K+B$ a. $Z$ é um operador compacto e $\left\|(K+B)_{\left.\right|_{Z}}\right\| \leq \frac{1}{\left\|K^{-1}\right\|}$. Pela Proposição 53 a restrição $B_{l_{Z}}$ de $B$ a $Z$ é um isomorfismo. Uma contradição, pois $\operatorname{dim}(R(B))<\infty$ e $\operatorname{dim}(Z)=\infty$.

Proposição 120. Sejam $X$ e $Y$ espaços de Banach. Um operador $T \in L(X, Y)$ é Fredholm se, e somente se, existem operadores $U, V \in L(X, Y), H \in \mathcal{F}(X)$ e $J \in \mathcal{F}(Y)$ tais que $U T=I_{X}-H$ e $T V=I_{Y}-J$, onde $\mathcal{F}(X)$ denota a classe de todos os operadores de posto finito de $X$ em $X$ e $\mathcal{F}(Y)$ denota a classe de todos os operadores de posto finito de $Y$ em $Y$. 
Prova. Pela Proposição $54 T$ admite a seguinte fatorização

$$
T=J_{R(T)}^{Y} T_{0} Q
$$

onde $T_{0}$ é um isomorfismo de $X / N(T)$ sobre $R(T), Q$ é a aplicação quociente canônica de $X$ em $X / N(T)$ e $J_{R(T)}^{Y}$ é a inclusão canônica de $R(T)$ em $Y$. Em suma, temos que

$$
\frac{X}{N(T)} \stackrel{H_{9}}{\rightarrow} X \stackrel{Q}{\rightarrow} \frac{X}{N(T)} \stackrel{T_{3}}{\rightarrow} R(T) \stackrel{J_{R(T)}^{Y}}{\rightarrow} Y \stackrel{J_{Q}}{\rightarrow} R(T) .
$$

Segue pela. Proposição 158 que existe $J_{o} \in L(Y, R(T))$ com $Q H_{o}=I_{\frac{X}{N(T)}}$ e, pela Proposição 159, que existe $H_{o} \in L\left(\frac{X}{N(T)}, X\right)$ com $J_{o} J_{R(T)}^{Y}=I_{R(T)}$. Se $H=I_{X}-H_{\circ} Q, S=$ $I_{Y}-J_{R(T)}^{Y} J_{o}$ e $U=V=H_{o} T_{o}^{-1} J_{o}$, então

$$
\begin{gathered}
U T=H_{o} T_{o}^{-1} J_{o} J_{R(T)}^{Y} T_{o} Q=H_{o} Q=I_{X}-H \\
T V=J_{R(T)}^{Y} T_{o} Q H_{o} T_{o}^{-1} J_{o}=J_{R(T)}^{Y} J_{o}=I_{Y}-S .
\end{gathered}
$$

Dado $x \in R(H)$, temos que existe $e \in X$ tal que

$$
\begin{gathered}
T\left(e-H_{o} Q(e)\right)=T(e)-T H_{o} Q(e)=T(e)-J_{R(T)}^{Y} T_{o} Q H_{o} Q(e)= \\
=T(e)-J_{R(T)}^{Y} T_{o} Q(e)=T(e)-T(e)=0 .
\end{gathered}
$$

Portanto, $R(H) \subset N(T)$ e então $\operatorname{dim}(R(H))<\infty$. Além disso, se $y \in N(S)$ então $y-J_{R(T)}^{Y} J_{o}(y)=0$ implicando que $y=J_{R(T)}^{Y} J_{o}(y)=T V(y) \in R(T)$. Logo, $N(S) \subset R(T)$. Por outro lado, se $y \in R(T)$ então existe $x \in X$ tal que $y=T(x)$, implicando que

$$
S(y)=S T(x)=T(x)-J_{R(T)}^{Y} J_{0} T(x)=T(x)-T(x)=0
$$

ou seja, $R(T) \subset N(S)$. Assim, $N(S)=R(T)$.

Reciprocamente, suponhamos que existem operadores lineares $U, V \in L(Y, X), H \in$ $\mathcal{F}(X)$ e $J \in \mathcal{F}(Y)$ tais que

$$
U T=I_{X}-H \text { e } T V=I_{Y}-J
$$

Dado $n \in N\left(I_{X}-H\right)$ temos que $n=H(n)$, implicando que $N\left(I_{X}-H\right) \subset H(X)$. Como $\operatorname{dim}(H(X))<\infty$ então

$$
\operatorname{dim}\left(N\left(I_{X}-H\right)\right)<\infty
$$

Se $x \in N(T)$ então $T(x)=0$, implicando por (2.29) que

$$
\left(I_{X}-H\right)(x)=U T(x)=U(0)=0 .
$$


Portanto $x \in N\left(I_{X}-H\right)$, implicando que $N(T) \subset N\left(I_{X}-H\right)$. Segue de (2.30) que

$$
\operatorname{dim}(N(T))<\infty
$$

Mostraremos agora que a imagem $R(T)$ de $T$ é um subespaço fechado e possui codimensão finita em $Y$. De fato, temos que $Y=R\left(I_{Y}-J\right)+R(J)$, implicando pelo Lema 117 que

$$
\operatorname{codim}\left(R\left(I_{Y}-J\right)\right)<\infty \text { em } Y,
$$

pois $\operatorname{dim}(R(J))<\infty$ por hipótese. Como $I_{Y}$ é um isomorfismo e $\operatorname{dim}(R(J))<\infty$ então segue do Lema 119 que

$$
R\left(I_{Y}-J\right) \text { é fechado. }
$$

Além disso, temos que $R\left(I_{Y}-J\right)=R(T V) \subset R(T) \subset Y$, implicando, pelo Lema 118, que

$$
\operatorname{codim}\left(R\left(I_{Y}-J\right)\right)<\infty \text { em } R(T) .
$$

Segue de (2.32) e (2.33) pela Proposição 9 que $R\left(I_{Y}-J\right)$ é um subespaço complementado de dimensão finita em $Y$. Logo, existe um subespaço de dimensão finita $M$ de $X$ tal que

$$
Y=R\left(I_{Y}-J\right)+M
$$

Por (2.34) e (2.35) existe um subespaço de dimensão finita $M_{\circ}$ de $R(T)$ tal que

$$
R(T)=R\left(I_{Y}-J\right)+M_{o}
$$

Segue de (2.33) e (2.36), pela Proposição 3, que a imagem $R(T)$ de $T$ é um subespaço fechado. Por (2.36) e (2.32) temos que

$$
\operatorname{codim}(R(T))<\infty \text { em } Y \text {. }
$$

De (2.37) e (2.31) obtemos que $T \in \Phi(X, Y)$.

Na álgebra de Calkin $L(X) / K(X)$, onde $K(X)$ é o ideal de todos os operadores compactos sobre um espaços de Banach $X$, consideremos a aplicação quociente $Q: L(X) \rightarrow$ $\frac{L(X)}{K(X)}$ definida por $Q(T)=[T], \forall T \in L(X)$. Neste caso, consideraremos que $\left[T_{1}\right]=\left[T_{2}\right]$ se, e somente se, $T_{1}-T_{2} \in K(E)$. É definida a seguinte lei interna: se $T, S \in L(X)$, então $[T] \odot[S]=[T S]$. Tal lei está bem definida. Com efeito, sejam $\left[T_{1}\right]=\left[T_{2}\right]$ e $\left[S_{1}\right]=\left[S_{2}\right]$ em $L(X) / K(X)$. Temos que $T_{1}-T_{2}=K_{1} \in K(X), \quad S_{1}-S_{2}=K_{2} \in K(X)$. Então 
$T_{1}=K_{1}+T_{2}$ e $S_{2}=S_{1}-K_{2}$, implicando que $T_{1} S_{1}=K_{1} S_{1}+T_{2} S_{1}$ e $T_{2} S_{2}=T_{2} S_{1}-T_{2} K_{2}$ e daí segue que $T_{1} S_{1}-T_{2} S_{2}=K_{1} S_{1}+T_{2} K_{2} \in K(X)$, pois $K(X)$ é um ideal de operadores. Assim, $\left[T_{1} S_{1}\right]=\left[T_{2} S_{2}\right]$.

Proposição 121. Seja $X$ um espaço de Banach. Um operador $T \in L(X)$ é Fredholm se, e somente se, $[T]$ é inversivel em $L(X) / K(X)$.

Prova. Segue da Proposição 120 que existem operadores $U, V \in L(X), H, J \in \mathcal{F}(X)$ tais que

$$
U T=I_{X}-H \text { e } T V=I_{X}-J
$$

Observemos que $\left[I_{X}-H\right]=\left[I_{X}\right]$, pois $I_{X}-H-I_{X}=H \in K(X)$. Analogamente, $\left[I_{X}-J\right]=\left[I_{X}\right]$. Assim

$$
\begin{gathered}
{[U] \odot[T]=[U T]=\left[I_{X}-H\right]=[I]} \\
{[T] \odot[V]=[T V]=\left[I_{X}-J\right]=\left[I_{X}\right]}
\end{gathered}
$$

Sendo $I_{X}$ o elemento identidade na álgebra de Calkin $L(X) / K(X)$, segue que $[T]$ é inversível nesse espaço.

Reciprocamente, se $[T]$ é inversível em $L(X) / K(X)$ então existem operadores $U, V \in$ $L(X)$ tais que

$$
\begin{aligned}
& {\left[I_{X}\right]=[T] \odot[V]=[T V] \Rightarrow T V=I_{X}-H} \\
& {\left[I_{X}\right]=[U] \odot[T]=[U T] \Rightarrow U T=I_{X}-J}
\end{aligned}
$$

onde $H, J \in K(X)$. Como o operador identidade subtraído de um operador compacto é um operador de Fredholm (veja o Corolário 105) então segue da Proposição 120 que existem operadores $U_{o}, V_{o} \in L(X)$ e operadores $H_{o}, J_{o} \in \mathcal{F}(X)$ tais que

$$
\begin{gathered}
U_{o}(U T)=I_{X}-H_{o} \Rightarrow\left(U_{o} U\right) T=I_{X}-H_{o} \\
(T V) V_{o}=I_{X}-J_{o} \Rightarrow T\left(V V_{o}\right)=I_{X}-J_{o}
\end{gathered}
$$

implicando, pela Proposição 120 , que $T \in \Phi(X)$.

Observação 122. Se $A, B \in L(X)$ e $A B$ é um operador de Fredholm então ambos os $A$ e $B$ são operadores de Fredholm ou nem A nem B são operadores de Fredholm.

De acordo com o resultado da proposição anterior os operadores de Fredholm possuem imagem inversível, pela aplicação quociente canônica, em $L(X) / K(X)$. Se considerarmos 
que os elementos inversíveis de uma álgebra formam um grupo e que os fatores de um produto inversível são ou ambos inversíveis ou ambos não inversíveis então segue que se $A, B \in L(X)$ e $A B$ é um operador de Fredholm então ambos os $A$ e $B$ são operadores de Fredholm ou nem $A$ nem $B$ são operadores de Fredholm. A soma de um operador de Fredholm com um operador de posto finito é também um operador de Fredholm.

Observação 123. S'egue das considerações anteriores que se $X$ é um espaço de Banach então os operadores inessenciais são os elementos de $L(X)$ cuja imagem inversa na álgebra de Calkin $L(X) / K(X)$ pertence ao radical. 


\section{Teoria espectral e funções de operadores lineares}

Nesse capítulo apresentaremos resultados de teoria espectral de operadores lineares em preparação para o estudo das funções de um operador linear. O objetivo é apresentar o Teorema da aplicação espectral (veja Teorema 138) que será utilizado nas demonstrações dos resultados parciais do Problema 1 (apresentado na Introdução) para o caso de espaços de Banach $X$ e $Y$ tais que todo operador linear contínuo de $X$ em $Y$ é estritamente singular e também para o caso em que todo operador linear contínuo de $X$ em $Y$ é inessencial.

Definição 124. Sejam $X$ um espaço de Banach e $T \in L(X)$. O conjunto resolvente $\rho(T)$ de $T$ é o conjunto dos números complexos $\lambda$ para os quais $(\lambda I-T)^{-1}$ existe como um operador contínuo com domínio $X$. $O$ espectro $\sigma(T)$ de $T$ é o complemento algébrico de $\rho(T)$ em $\mathbb{C}$, isto é, $\sigma(T)=\mathbb{C}-\rho(T)$. A função $R_{T}: \rho(T) \rightarrow L(X)$ definida por $R_{T}(\lambda)=(\lambda I-T)^{-1}$ é chamada função resolvente de $T$.

Lema 125. Sejam $X$ um espaços de Banach e $T \in L(X)$. O conjunto resolvente $\rho(T)$ de $T$ é aberto e a função resolvente $R_{T}$ é analítica em $\rho(T)$.

Prova. Fixemos $\lambda$ em $\rho(T)$ e consideremos $\mu \in \mathbb{C}$ tal que

$$
|\mu|<\frac{1}{\left|R_{T}(\lambda)\right|}
$$

Mostraremos que $\lambda+\mu \in \rho(T)$ e assim concluiremos que $\rho(T)$ é um conjunto aberto. Uma analogia com a teoria de séries geométricas sugere que se

$$
(\lambda+\mu) I-T=\mu I+(\lambda I-T)
$$


possui uma inversa, onde $I$ é o operador identidade sobre $X$, então esta inversa é dada pela série

$$
S(\mu)=\sum_{k=0}^{\infty}(-\mu)^{k}(\lambda I-T)^{-(k+1)}=\sum_{k=0}^{\infty}(-\mu)^{k} R_{T}(\lambda)^{k+1}
$$

ou seja,

$$
\begin{aligned}
& S(\mu)=(\lambda I-T)^{-1}-\mu\left[(\lambda I-T)^{-1}\right]^{2}+\mu^{2}\left[(\lambda I-T)^{-1}\right]^{3}-\cdots \\
& S(\mu)=(\lambda I-T)^{-1} \underbrace{\left[I-\mu(\lambda I-T)^{-1}+\left(\mu(\lambda I-T)^{-1}\right)^{2}-\cdots\right]}_{W(\mu)}
\end{aligned}
$$

Segue de (3.1) que $\left|\mu(\lambda I-T)^{-1}\right|<1$ e, portanto, a série

$$
|I|+\left|\mu(\lambda I-T)^{-1}\right|+\left|\mu(\lambda I-T)^{-1}\right|^{2}+\cdots
$$

converge e, assim, também converge a série $W(\mu)$. Logo, $S(\mu)$ converge.

Além disso, $S(\mu)$ comuta com $T$. Para verificar isto, basta mostrar que $T$ comuta com $(\lambda I-T)^{-1}$. Observemos que

$$
\begin{gathered}
(\lambda I-T)^{-1}(\lambda I-T)=I \\
(\lambda I-T)^{-1} \lambda-(\lambda I-T)^{-1} T=I \\
(\lambda I-T)^{-1} T=(\lambda I-T)^{-1} \lambda-I
\end{gathered}
$$

e por outro lado,

$$
\begin{gathered}
(\lambda I-T)(\lambda I-T)^{-1}=I \\
\lambda(\lambda I-T)^{-1}-T(\lambda I-T)^{-1}=I \\
T(\lambda I-T)^{-1}=\lambda(\lambda I-T)^{-1}-I .
\end{gathered}
$$

Segue de (3.5) e (3.6) que $T(\lambda I-T)^{-1}=(\lambda I-T)^{-1} T$.

Mostraremos que $S(\mu)$ é o inverso de $(\lambda+\mu) I-T$. De fato,

$$
\begin{gathered}
{[(\lambda+\mu) I-T] S(\mu)=(\lambda I-T) S(\mu)+\mu S(\mu)=} \\
=(\lambda I-T) \sum_{k=0}^{\infty}(-\mu)^{k}\left[(\lambda I-T)^{-1}\right]^{k+1}+\mu \sum_{k=0}^{\infty}(-\mu)^{k}\left[(\lambda I-T)^{-1}\right]^{k+1}= \\
=\sum_{k=0}^{\infty}(-\mu)^{k}(\lambda I-T)^{-k}-\sum_{k=0}^{\infty}(-\mu)^{k+1}(\lambda I-T)^{-k+1}= \\
=\sum_{k=0}^{\infty}\left[(-\mu) R_{T}(\lambda)\right]^{k}-\sum_{k=0}^{\infty}\left[(-\mu) R_{T}(\lambda)\right]^{k+1}=I .
\end{gathered}
$$

Como $S(\mu)$ comuta com $T$ então também ocorre que

$$
S^{\prime}(\mu)[(\lambda+\mu) I-T]=I .
$$


Portanto, $\lambda+\mu \in \rho(T)$ e, deste modo, $\rho(T)$ é um conjunto aberto. Além disso, mostramos que $R_{T}(\lambda+\mu)=S(\mu)$ e é uma função analítica na vizinhança $|\mu|<\left|R_{T}(\lambda)\right|^{-1}$ do ponto $\mu=0$, pois

$$
S(\mu)=\sum_{k=0}^{\infty}(-\mu)^{k}(\lambda I-T)^{-(k+1)}=\sum_{k=0}^{\infty}(-\mu)^{k} R_{T}(\lambda)^{k+1}
$$

é uma série de potências e, deste modo, uma função analítica.

Corolário 126. Se $d(\lambda)$ é a distância de $\lambda$ ao espectro $\sigma(T)$ de um operador $T \in L(X)$, onde $X$ é um espaço de Banach, então $\left|R_{T}(\lambda)\right| \geq \frac{1}{d(\lambda)}$. Deste modo, $\left|R_{T}(\lambda)\right| \rightarrow \infty$ quando $d(\lambda) \rightarrow 0$ e o conjunto resolvente é o domínio natural de analiticidade de $R_{T}(\lambda)$.

Prova. Vimos na prova do Lema 125 que se $|\mu|<\left|R_{T}(\lambda)\right|^{-1}$ então $\lambda+\mu \in \rho(T)$. Logo

$$
d(\lambda) \geq\left|R_{T}(\lambda)\right|^{-1}
$$

pois se $d(\lambda)<\left|R_{T}(\lambda)\right|^{-1}$, isto é, se $\inf \{|\mu-\lambda|: \mu \in \sigma(T)\}<\left|R_{T}(\lambda)\right|^{-1}$ então existe $\mu_{o} \in \sigma(T)$ tal que $\left|\mu_{o}-\lambda\right|<\left|R_{T}(\lambda)\right|^{-1}$. Então pelo resultado do Lema 125 temos que

$$
\mu_{o}=\left(\mu_{o}-\lambda\right)+\lambda \in \rho(T)
$$

uma contradição.

Proposição 127. Sejam $X$ é um espaço vetorial normado, I o operador identidade sobre $X$ e $T \in L(X)$ tal que $|\lambda|>\|T\|,(\lambda I-T)^{-1}$ existe, é contínuo e

$$
(\lambda I-T)^{-1}(x)=\sum_{n=1}^{\infty} \frac{T^{n-1}(x)}{\lambda^{n}}
$$

para cada $x \in R(\lambda I-T)$. Se $X$ é completo $e|\lambda|>\|T\|$ então $\lambda \in \rho(T)$. Neste caso,

$$
(\lambda I-T)^{-1}=R_{T}(\lambda)=\sum_{n=1}^{\infty} \frac{T^{n-1}}{\lambda^{n}} .
$$

Prova. Se $|\lambda|>\|T\|$ temos que

$$
\begin{aligned}
\|(\lambda I-T)(x)\| & =\|\lambda(x)-T(x)\| \\
& \geq|\lambda|\|x\|-\|T(x)\| \\
& \geq(|\lambda|-\|T\|)\|x\|
\end{aligned}
$$


e portanto $(\lambda I-T)^{-1}$ existe e é um operador contínuo para $|\lambda|>\|T\|$. Temos que $\|T / \lambda\|<1$ e então aplicando a. Proposição 52 a.o operador $A=\frac{T}{\lambda}$ temos que

$$
\left(I-\frac{T}{\lambda}\right)^{-1}=I+\frac{T}{\lambda}+\frac{T^{2}}{\lambda^{2}}+\cdots+\frac{T^{n}}{\lambda^{n}}+\cdots
$$

e daí,

$$
\lambda(\lambda I-T)^{-1}=\left(\frac{\lambda I-T}{\lambda}\right)^{-1}=I+\frac{T}{\lambda}+\cdots+\frac{T^{n}}{\lambda^{n}}+\cdots
$$

Portanto,

$$
(\lambda I-T)^{-1}=\frac{I}{\lambda}+\frac{T}{\lambda^{2}}+\cdots+\frac{T^{n}}{\lambda^{n+1}}+\cdots=\sum_{n=1}^{\infty} \frac{T^{n-1}}{\lambda^{n}}
$$

Logo, $\lambda \in \rho(T)$ se $|\lambda|>\|T\|$.

Corolário 128. Se $T \in L(X)$ e $X$ é um espaço de Banach então $|\lambda| \leq\|T\|, \forall \lambda \in \sigma(T)$.

Prova. A prova é uma conseqüência imediata da Proposição 127.

Lema 129. Sejam $X$ um espaço vetorial normado e $T \in L(X)$. O conjunto fechado $\sigma(T)$ é limitado e não-vazio. Além disso, $\sup |\sigma(T)|=\lim _{n \rightarrow \infty} \sqrt[n]{\left\|T^{n}\right\|} \leq\|T\|$. Para $|\lambda|>\sup |\sigma(T)|$ a série $R_{T}(\lambda)=\sum_{n=0}^{\infty} \frac{T^{n}}{\lambda^{n+1}}$ converge em $L(X)$.

Prova. Seja $f(\lambda)=\sum_{n=0}^{\infty} \frac{T^{n}}{\lambda^{n+1}}$. Esta série possui domínio de convergência $D=\{\lambda$ : $\left.|\lambda|>\lim _{n \rightarrow \infty} \lim \sup \sqrt[n]{\left\|T^{n}\right\|}\right\}$. Seja $I$ o operador identidade sobre $X$. Temos que $R_{T}(\lambda)=$ $f(\lambda)=\sum_{n=0}^{\infty} \frac{T^{n}}{\lambda^{n+1}}$ para $\lambda \in D$, pois

$$
\begin{gathered}
f(\lambda)(\lambda I-T)=\left(\sum_{n=0}^{\infty} \frac{T^{n}}{\lambda^{n+1}}\right)(\lambda I-T)= \\
=\lambda\left(\sum_{n=0}^{\infty} \frac{T^{n}}{\lambda^{n+1}}\right)-\left(\sum_{n=0}^{\infty} \frac{T^{n}}{\lambda^{n+1}}\right) T= \\
=\sum_{n=0}^{\infty} \frac{T^{n}}{\lambda^{n}}-\sum_{n=0}^{\infty} \frac{T^{n+1}}{\lambda^{n+1}}=I=(\lambda I-T) f(\lambda),
\end{gathered}
$$

para $\lambda \in D$.

Vimos no Corolário 126 que $\rho(T)$ é o domínio natural de analiticidade de $R_{T}(\lambda)$ e, portanto, sua série de Laurent $\sum_{n=0}^{\infty} \frac{T^{n}}{\lambda^{n+1}}$ possui domínio de convergência $|\lambda|>\sup |\sigma(T)|$. Deste modo, 


$$
\sup |\sigma(T)|=\lim \sup \sqrt[n]{\left\|T^{n}\right\|}
$$

Agora mostraremos que sup $|\sigma(T)| \leq \lim \inf \sqrt[n]{\left\|T^{n}\right\|}$ e com isto concluiremos que existe o limite $\lim _{n \rightarrow \infty} \sqrt[n]{\left\|T^{n}\right\|}$ e é igual a sup $|\sigma(T)|$.

De fato, suponhamos que $\lambda \in \sigma(T)$ é tomado de modo arbitrário. Então

$$
\lambda^{n} \in \sigma\left(T^{n}\right)
$$

e isto decorre da fatorização

$$
\lambda^{n} I-T^{n}=(\lambda I-T) P_{n}(T)=P_{n}(T)(\lambda I-T)
$$

onde $P_{n}(T)=\sum_{k=1}^{n} \lambda^{n-k} I T^{k-1}$, pois se $\lambda^{n} I-T^{n}$ possui uma inversa contínua então o mesmo ocorre com $\lambda I-T$, o que contradiz $\lambda \in \sigma(T)$. Logo, verifica-se 3.12. Deste modo, pelo Corolário 128 , temos que

$$
|\lambda|^{n} \leq\left\|T^{n}\right\|
$$

e então $\sup |\sigma(T)| \leq \sqrt[n]{\left\|T^{n}\right\|}$. Segue daí que

$$
\sup |\sigma(T)| \leq \lim \inf \sqrt[n]{\left\|T^{n}\right\|}
$$

Mostraremos que $\sigma(T) \neq \emptyset$. De fato, se $\sigma(T)=\emptyset$ então $R_{T}(\lambda)$ é uma função analítica e limitada em $\mathbb{C}$ então temos, pelo Teorema de Liouville, que $R_{T}(\lambda)$ é constante. Segue do Corolário 126 que $\left|R_{T}(\lambda)\right| \rightarrow 0$ quando $|\lambda| \rightarrow+\infty$. Então $R_{T}(\lambda)=0$ é o operador nulo. Como $R_{T}(\lambda)$ é sobrejetora sobre $X$ temos que $X=\{0\}$, que é o caso trivial. Logo, $\sigma(T) \neq \emptyset$.

Observação 130. Seja $X$ um espaço vetorial normado. Se $T \in L(X)$ e se a série $\sum_{n=1}^{\infty} \frac{T^{n-1}}{\lambda^{n}}$ converge em $L(X)$ para algum valor de $\lambda$ então $\lambda \in \rho(T)$ e o operador definido pela série é $R_{T}(\lambda)$, pois se $A=\sum_{n=1}^{\infty} \frac{T^{n-1}}{\lambda^{n}}$ é fácil verificar que $(\lambda I-T) A=A(\lambda I-T)=I$.

Definição 131. A quantidade $r_{\sigma}(T)=\sup |\sigma(T)|=\lim _{n \rightarrow \infty} \sqrt[n]{\|T\|}$ é chamada de raio espectral do operador $T$.

Lema 132. Sejam $X$ um espaço vetorial normado e $T \in L(X)$. Então para qualquer par $\lambda, \mu \in \rho(T)$ temos que

$$
R_{T}(\lambda)-R_{T}(\mu)=(\mu-\lambda) R_{T}(\lambda) R_{T}(\mu) .
$$

Esta identidade é conhecida como equação resolvente. 
Prova. Temos que

$$
(\mu I-T)(\lambda I-T)\left[R_{T}(\lambda)-R_{T}(\mu)\right]=(\mu I-T)-(\lambda I-T)=(\mu-\lambda) I .
$$

Multiplicando ambos os membros desta equação por $R_{T}(\lambda) R_{T}(\mu)$ obtemos

$$
R_{T}(\lambda)-R_{T}(\mu)=(\mu-\lambda) R_{T}(\lambda) R_{T}(\mu) .
$$

Definição 133. Por $\mathcal{F}(T)$ denotaremos a família de todas as funções $f$ que são analíticas sobre alguma vizinhança de $\sigma(T)$ de um operador $T \in L(X)$, onde $X$ é um espaço de Banach.

Definição 134. Seja $f \in \mathcal{F}(T)$ e seja $U$ um conjunto aberto cujo bordo $B$ consiste de um número finito de curvas de Jordan retificáveis, orientadas no sentido anti-horário. Suponha que $U \supseteq \sigma(T)$ e que $U \cup B$ está contido no dominio de analiticidade de $f$. Então o operador $f(T)$ é definido pela equação

$$
f(T)=\frac{1}{2 \pi i} \int_{B} f(\lambda) R_{T}(\lambda) d \lambda .
$$

Nos próximos resultados utilizaremos as seguintes observações:

\section{Observação 135 .}

Seja $n \in \mathbb{Z}$ e $\gamma$ uma circunferência de centro $z_{o}$ e raio $r$. Calcularemos a integral $\int_{\gamma}\left(z-z_{0}\right)^{n} d z$.

A equação paramétrica de $\gamma$ é

$$
z(t)=z_{o}+r(\cos (t)+i \operatorname{sen}(t)), \quad 0 \leq t \leq 2 \pi .
$$

Temos que

$$
z^{\prime}(t)=r(-\operatorname{sen}(t)+i \cos (t))=i r(\cos (t)+i \operatorname{sen}(t)), \quad 0 \leq t \leq 2 \pi .
$$

Então

$$
\begin{gathered}
\int_{\gamma}\left(z-z_{0}\right)^{n} d z=\int_{0}^{2 \pi}\left(z(t)-z_{0}\right)^{n} z^{\prime}(t) d t= \\
=\int_{0}^{2 \pi} r^{n}(\cos (t)+i \operatorname{sen}(t))^{n} i r(\cos (t)+i \operatorname{sen}(t)) d t=
\end{gathered}
$$




$$
\begin{gathered}
=i r^{n+1} \int_{0}^{2 \pi}(\cos (t)+i \operatorname{sen}(t))^{n+1} d t= \\
=i r^{n+1} \int_{0}^{2 \pi}[\cos ((n+1) t)+i \operatorname{sen}((n+1) t)] d t .
\end{gathered}
$$

Portanto,

$$
\int_{\gamma}\left(z-z_{0}\right)^{n} d z=i r^{n+1} \int_{0}^{2 \pi}[\cos ((n+1) t)+i \operatorname{sen}((n+1) t)] d t .
$$

Mas,

$$
\int_{0}^{2 \pi} \cos (k t) d t=\int_{0}^{2 \pi} \operatorname{sen}(k t) d t=0, \text { se } k \neq 0
$$

e

$$
\int_{0}^{2 \pi} \cos (k t) d t=2 \pi \text { e } \int_{0}^{2 \pi} \operatorname{sen}(k t)=0, \text { se } k=0 .
$$

Então, por (3.18) e (3.19), temos em (3.17) que

$$
\int_{\gamma}\left(z-z_{0}\right)^{n} d z=\left\{\begin{array}{rll}
2 \pi i, & \text { se } & n=-1 \\
0, & \text { se } & n \neq-1
\end{array}\right.
$$

\section{Observação 136.}

Sejam $X$ um espaço de Banach e $T \in L(X)$. Sabemos que $\sigma(T)$ é limitado e $R_{T}(\lambda)=$ $\sum_{n=1}^{\infty} \frac{T^{n-1}}{\lambda^{n}}$ quando $|\lambda|>r_{\sigma}(T)$. Se $C$ é uma curva simples fechada, orientada no sentido anti-horário, contendo o círculo $|\lambda|=r_{\sigma}(T)$, podemos integrar $R_{T}(\lambda)$ termo a termo em torno da curva $C$ e obtemos, aplicando a Observação $135 \operatorname{com} z_{o}=0$, que

$$
\begin{gathered}
\int_{C} R_{T}(\lambda) d \lambda=\int_{C} \sum_{n=1}^{\infty} \frac{T^{n-1}}{\lambda^{n}} d \lambda= \\
=\sum_{n=1}^{\infty} \int_{C} \frac{T^{n-1}}{\lambda^{n}} d \lambda=\sum_{n=1}^{\infty} \int_{|\lambda|=r_{\sigma}(T)} \frac{T^{n-1}}{\lambda^{n}} d \lambda= \\
=\int_{|\lambda|=r_{\sigma}(T)} I \lambda^{-1} d \lambda+\int_{|\lambda|=r_{\sigma}(T)} T \lambda^{-2} d \lambda+\int_{|\lambda|=r_{\sigma}(T)} T^{2} \lambda^{-3} d \lambda+\cdots= \\
=2 \pi i I .
\end{gathered}
$$

Então,

$$
I=\frac{1}{2 \pi i} \int_{C} R_{T}(\lambda) d \lambda
$$


Analogamente, temos que $T^{k}=\frac{1}{2 \pi i} \int_{C} \lambda^{k} R_{T}(\lambda) d \lambda, \quad k=0,1,2, \ldots$ De fato, multiplicando ambos os membros da igualdade

$$
R_{T}(\lambda)=I \lambda^{-1}+T \lambda^{-2}+T^{2} \lambda^{-3}+\cdots, \quad|\lambda|>r_{\sigma}(T)
$$

por $\lambda^{k}, \quad(k=0,1,2,3, \ldots)$, temos que

$$
\lambda^{k} R_{T}(\lambda)=I \lambda^{k-1}+T \lambda^{k-2}+\cdots+T^{k-1} \lambda^{0}+T^{k} \lambda^{-1}+\cdots
$$

Integrando termo-a-termo obtemos

$$
\int_{C} \lambda^{k} R_{T}(\lambda) d \lambda=\int_{C} I \lambda^{k-1} d \lambda+\cdots+\int_{C} T^{k-1} d \lambda+\int_{C} T^{k} \lambda^{-1} d \lambda+\cdots=2 \pi i T^{k}
$$

Portanto,

$$
T^{k}=\frac{1}{2 \pi i} \int_{C} \lambda^{k} R_{T}(\lambda) d \lambda
$$

Proposição 137. Se $f, g \in \mathcal{F}(T)$ e $\alpha, \beta \in \mathbb{C}$ então

(i) $\alpha f+\beta g \in \mathcal{F}(T)$ e $\alpha f(T)+\beta g(T)=(\alpha f+\beta g)(T)$;

(ii) $f g \in \mathcal{F}(T)$ e $f(T) g(T)=(f g)(T)$;

(iii) Se $f$ possui a expansão em séries de potências $f(\lambda)=\sum_{k=0}^{\infty} \alpha_{k} \lambda^{k}$, válida em uma vizinhança de $\sigma(T)$ então $f(T)=\sum_{k=0}^{\infty} \alpha_{k} T^{k}$;

(iv) $f \in \mathcal{F}\left(T^{*}\right)$ e $f\left(T^{*}\right)=f(T)^{*}$.

Prova. (i) É imediata.

(ii) Também é imediato que $f g \in \mathcal{F}(T)$; sejam $U_{1}$ e $U_{2}$ duas vizinhanças de $\sigma(T)$ cujos bordos $B_{1}$ e $B_{2}$ consistem de um número finito de curvas de Jordan retificáveis e, suponhamos que $U_{1} \cup B_{1} \subset U_{2}$. Suponhamos também que $U_{2} \cup B_{2}$ está contida em uma região comum de analiticidade de $f$ e $g$. Então, pelo Lema 132, temos que

$$
\begin{gathered}
f(T) g(T)=\left(\frac{1}{2 \pi i} \int_{B_{1}} f(\lambda) R_{T}(\lambda) d \lambda\right)\left(\frac{1}{2 \pi i} \int_{B_{2}} g(\mu) R_{T}(\mu) d \mu\right)= \\
=-\frac{1}{4 \pi^{2}}\left(\int_{B_{1}} f(\lambda) R_{T}(\lambda) d \lambda\right)\left(\int_{B_{2}} g(\mu) R_{T}(\mu) d \mu\right)= \\
=-\frac{1}{4 \pi i} \int_{B_{1}} \int_{B_{2}} f(\lambda) g(\mu) R_{T}(\lambda) R_{T}(\mu) d \mu d \lambda=
\end{gathered}
$$




$$
\begin{gathered}
=-\frac{1}{4 \pi^{2}} \int_{B_{1}} \int_{B_{2}} f(\lambda) g(\mu) \frac{R_{T}(\lambda)-R_{T}(\mu)}{\lambda-\mu} d \mu d \lambda= \\
=-\frac{1}{4 \pi^{2}} \int_{B_{1}} f(\lambda) R_{T}(\lambda) \underbrace{\left(\int_{B_{2}} \frac{g(\mu)}{\mu-\lambda} d \mu\right)}_{2 \pi i g(\lambda)} d \lambda+\frac{1}{4 \pi^{2}} \int_{B_{2}} g(\mu) R_{T}(\mu)\left(\int_{B_{1}} \frac{f(\lambda)}{\mu-\lambda} d \lambda\right) d \mu= \\
=-\frac{1}{4 \pi^{2}} \int_{B_{1}} f(\lambda) 2 \pi i g(\lambda) R_{T}(\lambda) d \lambda= \\
=\frac{1}{2 \pi i} \int_{B_{1}} f(\lambda) g(\lambda) R_{T}(\lambda) d \lambda=(f g)(T) .
\end{gathered}
$$

Isto prova (ii).

(iii) Notemos que a série de potências $\sum_{k=0}^{\infty} \alpha_{k} \lambda^{k}$ converge uniformemente sobre o círculo $C=\{\lambda:|\lambda| \leq r(T)+\varepsilon\}$ para $\varepsilon$ suficientemente pequeno. Conseqüentemente

$$
\begin{gathered}
f(T)=\frac{1}{2 \pi i} \int_{C}\left(\sum_{k=0}^{\infty} \alpha_{k} \lambda^{k}\right) R_{T}(\lambda) d \lambda= \\
=\frac{1}{2 \pi i} \sum_{k=0}^{\infty} \alpha_{k} \int_{C} \lambda^{k}\left(\sum_{j=0}^{\infty} \frac{T^{j}}{\lambda^{j+1}}\right) d \lambda= \\
=\sum_{k=0}^{\infty} \alpha_{k} T^{k},
\end{gathered}
$$

pelo Lema 129 , pois $T^{k}=\frac{1}{2 \pi i} \int_{C} \lambda^{k} R_{T}(\lambda) d \lambda, \quad k=1,2,3, \ldots$ em virtude da Observação 136, Fórmula (3.22).

Finalmente estamos em condições de enunciar e provar o principal resultado desse capítulo.

Teorema 138 (Teorema da aplicação espectral). Sejam $X$ um espaço de Banach e $T \in L(X)$. Se $f \in \mathcal{F}(T)$ então $f(\sigma(T))=\sigma(f(T))$.

Prova. Seja $\lambda \in \sigma(T)$. Definimos a função $g: D(f) \subset \mathbb{C} \rightarrow \mathbb{C}$ por

$$
g(\xi)=\frac{f(\lambda)-f(\xi)}{\lambda-\xi}, \forall \xi \in D(f) .
$$

Pela Proposição 137 segue que

$$
f(\lambda) I_{X}-f(T)=\left(\lambda I_{X}-T\right) g(T)
$$


Suponhamos, por contradição, que $f(\lambda) \notin \sigma(f(T))$. Nesse caso, temos que $f(\lambda) I_{X}-$ $f(T)$ possui uma inversa contínua $A$ definida em todos os pontos e, daí, o operador $g(T) A$ é uma inversa contínua para $\lambda I_{X}-T$, contradizendo a hipótese $\lambda \in \sigma(T)$. Logo, $f(\lambda) \in \sigma(f(T))$. Assim, fica provado que $f(\sigma(T)) \subset \sigma(f(T))$.

Reciprocamente, seja $\mu \in \sigma(f(T))$. Suponhamos, por contradição, que $\mu \notin f(\sigma(T))$. Nesse caso, a função

$$
h(\xi)=(f(\xi)-\mu)^{-1}
$$

pertence a. $\mathcal{F}(T)$, pois $\mu \notin f(\sigma(T))$, implicando que $\mu \neq f(\xi), \forall \xi \in \sigma(T)$, que por sua vez implica que $f(\xi)-\mu \neq 0, \forall \xi \in \sigma(T)$.

Então pela. Proposição 137 temos que

$$
h(T)\left(f(T)-\mu I_{X}\right)=I_{X}
$$

o que contradiz a hipótese $\mu \in \sigma(f(T))$. Logo, $\mu \in f(\sigma(T))$, isto é, $\sigma(f(T)) \subset f(\sigma(T))$.

Assim, $\sigma(f(T))=f(\sigma(T))$. 
Capítulo 4

\section{Subespaços complementados na soma de espaços de Banach}

Nesse capítulo exibiremos o resultado obtido por Wojtaszczyk, P. [34], em 1978, que dá uma resposta positiva ao Problema 1 apresentado na Introdução para o caso de espaços de Banach $X$ e $Y$ tais que $S S(X, Y)=L(X, Y)$. O resultado estudado em [20] mostra que nesse caso para todo subespaço complementado $Z$ em $X \oplus Y$ existe um isomorfismo bijetor $\tau_{o} \in L(X \oplus Y)$ e subespaços $X_{o}$ complementado em $X$ e $Y_{o}$ complementado em $Y$ tais que $\tau_{o}(Z)=X_{o} \oplus Y_{o}$.

O lema a seguir é um resultado elementar da Álgebra Linear e portanto omitiremos sua demonstração.

Lema 139. Sejam $X$ e $Y$ espaços de Banach e $V$ um subespaço de dimensão finita de $X \oplus Y$. Então $V=V_{X} \oplus V_{Y}$, onde $V_{X}$ e $V_{Y}$ são subespaços de dimensão finita de $X$ e de $Y$, respectivamente.

Lema 140. Se $V_{1}$ e $V_{2}$ são dois subespaços fechados de um espaço de Banach $X$ com. $\operatorname{dim}\left(X / V_{1}\right)=\operatorname{dim}\left(X / V_{2}\right)<\infty$ então existe um isomorfismo de $X$ em $X$ que leva $V_{1}$ sobre $V_{2}$ (o isomorfismo pode ser escolhido sendo o operador identidade sobre $V_{1} \cap V_{2}$ desde que $\left.\operatorname{dim}\left[V_{1} /\left(V_{1} \cap V_{2}\right)\right]=\operatorname{dim}\left[V_{2} /\left(V_{1} \cap V_{2}\right)\right]<\infty\right)$.

Prova. Temos, pelo Lema 78, que existem subespaços de dimensão finita $M$ de $V_{1}$ e $N$ de $V_{2}$ tais que

$$
V_{1}=\left(V_{1} \cap V_{2}\right) \oplus N, \text { onde } \operatorname{dim}(N)<\infty
$$




$$
V_{2}=\left(V_{1} \cap V_{2}\right) \oplus M, \text { onde } \operatorname{dim}(M)<\infty .
$$

Como $\operatorname{dim}\left(X / V_{1}\right)=\operatorname{dim}\left(X / V_{2}\right)<\infty$ então $\operatorname{codim}\left(V_{1}\right)=\operatorname{codim}\left(V_{2}\right)<\infty$ em $X$ então $V_{1}$ e $V_{2}$ são complementados em $X$ pelos subespaços de dimensão finita $W_{1}$ e $W_{2}$, respectivamente, pois $V_{1}$ e $V_{2}$ são fechados. Ou seja,

$$
\begin{aligned}
& U=V_{1} \oplus W_{1}=\left(V_{1} \cap V_{2}\right) \oplus\left[N \oplus W_{1}\right] \\
& U=V_{2} \oplus W_{2}=\left(V_{1} \cap V_{2}\right) \oplus\left[M \oplus W_{2}\right]
\end{aligned}
$$

Além disso, $\operatorname{dim}\left(W_{1}\right)=\operatorname{dim}\left(W_{2}\right)<\infty$. Segue daí que $\operatorname{dim}(M)=\operatorname{dim}(N)<\infty$. Então existe um isomorfismo sobrejetor $\varphi: N \rightarrow M$. Por (4.1) consideremos as projeções $P_{1}: V_{1} \rightarrow V_{1} \cap V_{2}$ e $P_{2}: V_{1} \rightarrow N$. Seja $T: V_{1} \rightarrow V_{2}$ o operador linear definido por por $T\left(v_{1}\right)=P_{1}\left(v_{1}\right)+\varphi\left(P_{2}\left(v_{1}\right)\right), \forall v_{1} \in V_{1}$. Temos que

- $T$ é injetora.

Se $v_{1} \in N(T)$ então $T\left(v_{1}\right)=0$. Logo,

$$
0=T\left(v_{1}\right)=P_{1}\left(v_{1}\right)+\varphi\left(P_{2}\left(v_{1}\right)\right) .
$$

Portanto $P_{1}\left(v_{1}\right)=-\varphi\left(P_{2}\left(v_{1}\right)\right)$. Segue de (4.1) e (4.2) que $\varphi\left(P_{2}\left(v_{1}\right)\right)=0$ implicando que $P_{2}\left(v_{1}\right)=0$ e $P_{1}\left(v_{1}\right)=0$. Como $v_{1}=P_{1}\left(v_{1}\right)+P_{2}\left(v_{1}\right)$ então $v_{1}=0$. Assim, $N(T)=\{0\}$. - $T$ é sobrejetora.

Seja $v+m \in V_{2}$, onde $v \in V_{1} \cap V_{2}$ e $m \in M$. Então

$$
T\left(v+\varphi^{-1}(m)\right)=P_{1}\left(v+\varphi^{-1}(m)\right)+\varphi\left(P_{2}\left(v+\varphi^{-1}(m)\right)\right)=v+\varphi \varphi^{-1}(m)=v+m .
$$

- $T$ é contínuo, pois $\varphi, P_{1}$ e $P_{2}$ são contínuos.

Então segue do Teorema da aplicação aberta que $T$ é um isomorfismo.

Seja $\phi: W_{1} \rightarrow W_{2}$ um isomorfismo sobrejetor. Consideremos o operador linear $S$ : $X \rightarrow X$ definido por $S\left(v_{1}+w_{1}\right)=T\left(v_{1}\right)+\phi\left(w_{1}\right)$, onde $v_{1} \in V_{1}$ e $w_{1} \in W_{1}$. Temos que - $S$ é contínuo;

- $S$ é injetor, pois se $v_{1}+w_{1} \in N(L)$ então $T\left(v_{1}\right)=-\phi\left(w_{1}\right)$, implicando que $v_{1}=0$ e $w_{1}=0$. Portanto $N(L)=\{0\}$.

Pelo Teorema da aplicação aberta, $S$ é um isomorfismo sobre X.

Lema 141. Seja $X$ um espaço de Banach e $T: X \rightarrow X$ um operador de Fredholm de indice 0. Então existe um operador $H: X \rightarrow X$ de posto finito tal que $T+H: X \rightarrow X$ é um isomorfismo. 
Prova. Como $i(T)=0$ então

$$
\alpha(T)=\beta(T)<\infty .
$$

Como todo subespaço de dimensão finita é complementado então existe um subespaço fechado $U$ de $X$ tal que

$$
X=U \oplus N(T)
$$

Sendo $T$ um operador de Fredholm então $R(T)$ é um subespaço fechado de codimensão finita de $X$. Logo existe um subespaço fechado $V$ de $X$ tal que

$$
X=V \oplus R(T)
$$

e $\operatorname{dim}(V)=\operatorname{dim}(N(T))<\infty$

Então existe um isomorfismo $\varphi: N(T) \rightarrow V$. Além disso, segue de (4.6) que existe uma projeção $P: X \rightarrow N(T)$ tal que $R(P)=N(T)$. Então o operador $\varphi \circ P: X \rightarrow V$ tem posto finito pois $V$ é um subespaço de dimensão finita. Definimos $H=\varphi \circ P$. Seja $x \in N(T+H)$. Temos que

$$
0=(T+H)(x)=T(x)+H(x) .
$$

Portanto $T(x)=-H(x)$. Como $T(x) \in R(T)$ e $-H(x) \in R(H)$ e $R(H) \subset V$ então segue de (4.7) que $T(x)=-\varphi(P(x))=0$. Assim $x \in N(T)$. Como $\varphi$ é um isomorfismo então $\varphi(P(x))=0$ implica que $P(x)=0$ e portanto $x \in U$. Deste modo $x \in N(T) \cap U=$ $\{0\}$. Logo $x=0$.

Mostramos deste modo que $N(T+H)=\{0\}$ e portanto $\alpha(T+H)=0$.

Como $T$ é um operador de Fredholm e $H$ é um operador de posto finito então, pela Proposição 115 temos que $i(T+H)=i(T)=0$, implicando que $\beta(T+H)=0$. Portanto $T+H$ é um isomorfismo de $X$ sobre $X$.

Lema 142. Sejam $X$ e $Y$ espaços de Banach e consideremos o operador linear $T: X \oplus$ $Y \rightarrow X \oplus Y$, definido por $T(x, y)=\left(T_{\left.\right|_{X}}(x), T_{\left.\right|_{Y}}(y)\right), \forall(x, y) \in X \oplus Y$, onde $T_{\left.\right|_{X}} \in L(X)$ e $T_{\left.\right|_{Y}} \in L(Y)$. Suponhamos que $T_{\left.\right|_{X}}$ possui uma inversa contínua em $X$ e $T_{\left.\right|_{Y}}$ possui uma inversa contínua em $Y$. Então $T$ possui uma inversa contínua em $X \oplus Y$.

Prova. Sejam $A_{1}: X \rightarrow X$ e $A_{2}: Y \rightarrow Y$ as inversas de $T_{\left.\right|_{X}}$ e $T_{\left.\right|_{Y}}$, respectivamente. Então

$$
\begin{aligned}
& T_{\left.\right|_{X}} \circ A_{1}=A_{1} \circ T_{\left.\right|_{X}}=I_{X} \\
& T_{\left.\right|_{Y}} \circ A_{2}=A_{2} \circ T_{\left.\right|_{Y}}=I_{Y}
\end{aligned}
$$


Consideremos o operador linear $A: X \oplus Y \rightarrow X \oplus Y$ definido por

$$
A(x, y)=\left(A_{1}(x), A_{2}(y)\right), \forall x \in X, \forall y \in Y \text {. }
$$

Temos que

- $A$ está bem definido pois $A_{1}(x) \in X$ e $A_{2}(y) \in Y$;

- $A$ é contínuo; com efeito, consideremos em $X \oplus Y$ a norma $\|(x, y)\|=\max (\|x\|,\|y\|)$.

Como $A_{1}$ e $A_{2}$ são operadores lineares contínuos em $X$ e $Y$, respectivamente, segue que existem $M>0$ e $N>0$ tais que

$$
\begin{gathered}
\left\|A_{1}(x)\right\| \leq M\|x\|, \forall x \in X \\
\left\|A_{2}(y)\right\| \leq N\|y\|, \forall y \in Y .
\end{gathered}
$$

Então dado $x+y \in X \oplus Y$ arbitrário, temos que

$$
\|A(x, y)\|=\left\|\left(A_{1}(x), A_{2}(y)\right)\right\|=\max \left(\left\|A_{1}(x)\right\|,\left\|A_{2}(y)\right\|\right)
$$

Seja $M_{o}=\max \{M, N\}$. Temos que

$$
\begin{gathered}
\left\|A_{1}(x)\right\| \leq M_{o}\|x\|, \\
\left\|A_{2}(y)\right\| \leq M_{o}\|y\|,
\end{gathered}
$$

e isto implica que $\max \left(\left\|A_{1}(x)\right\|,\left\|A_{2}(y)\right\|\right) \leq \max \left(M_{o}\|x\|, M_{o}\|y\|\right)$. Assim, em (4.8) temos que

$$
\|A(x, y)\| \leq M_{o} \max (\|x\|,\|y\|)=M_{o}\|(x, y)\| .
$$

Portanto $A$ é um operador linear contínuo em $X \oplus Y$. Além disso, $A T(x, y)=A\left(T_{\left.\right|_{X}}(x), T_{\left.\right|_{Y}}(y)\right)=\left(A_{1} T_{I_{X}}(x), A_{2} T_{I_{Y}}(y)\right)=(x, y)=T A(x, y), \forall(x, y) \in X \oplus Y$.

Logo, $A$ é uma inversa contínua de $T$.

Definição 143. Um ponto $x \in \mathbb{R}$ é dito um ponto isolado se existe $\varepsilon>0$ tal que $B(x, \varepsilon) \cap$ $X=\{x\}$.

Observação 144. Se todos os pontos de um conjunto $X \subset \mathbb{R}^{2}$ são isolados então $X$ é enumerável. De fato, segue da Proposição 2 que existe um subconjunto $E \subset X$ enumerável $e$ denso em $X$. Como todo ponto de $X$ é isolado segue que $E=X$ e portanto $X$ é enumerável. 
Lema 145. Seja $X$ um espaço de Banach complexo e seja $P$ uma projeção em $X$. Seja $S: X \rightarrow X$ um operador estritamente singular e seja $Q=P+S$. Então o espectro $\sigma(Q)$ de $Q$ é enumerável e seus únicos possíveis pontos limites são 0 e 1 .

Prova. (i) $Q-\lambda I$ é um operador de Fredholm de índice $0, \forall \lambda \in \mathbb{C}-\{0,1\}$, onde $I$ é o operador identidade.

De fato, temos que

$$
Q-\lambda I=(P+S)-\lambda I=(P-\lambda I)+S
$$

O operador linear $P-\lambda I$ é contínuo e possui imagem fechada, pois $P$ é uma projeção em $X$. Se o operador $P-\lambda I$ é uma projeção em $X$ então não necessariamente $\alpha(P-\lambda I)<$ $\infty$. Mas $P-\lambda I$ é uma projeção em $X$ se, e somente se,

$$
P-\lambda I=(P-\lambda I)^{2}=(P-\lambda I)(P-\lambda I)=P^{2}-2 \lambda P+\lambda^{2} P=P-2 \lambda P+\lambda^{2} P,
$$

que implica $\left(\lambda^{2}-\lambda\right) x=0, \forall x \neq 0$ em $R(P)$ e portanto $\lambda(\lambda-1)=0$, implicando que $\lambda=0$ ou $\lambda=1$.

Portanto o operador linear $P-\lambda I$ é uma projeção se, e somente se, $\lambda=0$ ou $\lambda=1$. Se $\lambda \in \mathbb{C}-\{0,1\}$ então para $x \in N(P-\lambda I)$ temos que $(P-\lambda I)(x)=0$ implicando que $P(x)=\lambda x$ e então $P^{2}(x)=\lambda P(x)$, isto é, $P(x)=\lambda(\lambda x)$ e isto implica que $\lambda x=\lambda^{2} x$. Deste modo, $\left(\lambda^{2}-\lambda\right) x=0$.

Como $\lambda \in \mathbb{C}-\{0,1\}$ então $x=0$. Logo, $N(P-\lambda I)=\{0\}$ quando $\lambda \in \mathbb{C}-\{0,1\}$ e conseqüentemente temos que

$$
\alpha(P-\lambda I)=0, \forall \lambda \in \mathbb{C}-\{0,1\} .
$$

Além disso, $\operatorname{codim}(R(P-\lambda I))=0$ em $X$, ou equivalentemente, o operador $P-\lambda I$ é sobrejetor para $\lambda \in \mathbb{C}-\{0,1\}$. Com efeito, como $P: X \rightarrow X$ é uma projeção então $P^{*}: X^{*} \rightarrow X^{*}$ também o é, pois

$$
\left(P^{*}\right)^{2} x^{*}(x)=P^{*}\left(P^{*} x^{*}(x)\right)=P^{*} x^{*}(P(x))=x^{*} P^{2}(x)=x^{*} P(x)=P^{*} x^{*}(x), \forall x \in X .
$$

Então pelo que provamos anteriormente;

$$
N\left(P^{*}-\lambda I^{*}\right)=0, \forall \lambda \in \mathbb{C}-\{0,1\}
$$

implicando que

$$
\alpha\left(P^{*}-\lambda I^{*}\right)=0, \forall \lambda \in \mathbb{C}-\{0,1\}
$$


Segue pela. Proposição 77 e por (4.11) que

$$
\beta(P-\lambda I)=\alpha\left((P-\lambda I)^{*}\right)=\alpha\left(P^{*}-\lambda I^{*}\right)=0, \forall \lambda \in \mathbb{C}-\{0,1\}
$$

De (4.10) e (4.11) temos que $i(P-\lambda I)=0, \forall \lambda \in \mathbb{C}-\{0,1\}$. Pela Proposição 104 segue que

$$
i(Q-\lambda I)=i((P-\lambda I)+S)=i(P-\lambda I)=0
$$

(ii) $\sigma(Q)$ é enumerável.

O conjunto $\mathbb{C}-\{0,1\}$ é conexo (por caminhos) de acordo com a Proposição 15. Então quaisquer dois pontos de $\mathbb{C}-\{0,1\}$ estão contidos num mesmo subconjunto conexo de $\mathbb{C}-\{0,1\}$ e então numa mesma componente conexa de $\mathbb{C}-\{0,1\}$. Então, pela Proposição 107 , esses dois pontos arbitrários podem ser conectados por uma quantidade finita de bolas abertas que interceptam-se e onde $\alpha(Q-\lambda I)$ é constante, exceto possivelmente nos centros das bolas abertas. Portanto $\alpha(Q-\lambda I)$ é constante sobre $C-\{0,1\}$, exceto possivelmente num conjunto de pontos isolados. Vimos no Corolário 128 que $\sigma(Q) \subset\{\lambda:|\lambda| \leq\|Q\|\}$. Então $Q-\lambda I$ é inversível para $|\lambda|>\|Q\|$. Em tais pontos o operador $Q-\lambda I$ é injetor, implicando que $N(Q-\lambda I)=\{0\}$. Logo $\alpha(Q-\lambda I)=0$, exceto possivelmente sobre um conjunto de pontos isolados. Então

$$
0=i(Q-\lambda I)=\alpha(Q-\lambda I)-\beta(Q-\lambda I)=\beta(Q-\lambda I),
$$

exceto possivelmente em um conjunto de pontos isolados.

Desse modo, o espectro $\sigma(Q)$ de $Q$ está contido em um conjunto de pontos isolados, implicando, pela Proposição 144, que $\sigma(Q)$ é enumerável.

Definição 146. Seja $\Gamma$ uma curva simples e fechada em $\mathbb{C}$. Indicaremos por int $(\Gamma)$ o conjunto dos pontos interiores a curva $\Gamma$ e por ext $(\Gamma)$ o conjunto dos pontos exteriores a curva $\Gamma$.

A demonstração do teorema principal desta seção envolve a construção de uma projeção da forma $\frac{1}{2 \pi i} \int_{\Gamma} R_{Q}(\lambda) d \lambda$ e a partir daí outros operadores até que se obtém um isomorfismo e os subespaços complementados que se desejam. As demonstrações envolvem o caso em que os espaços de Banach em questão são complexos e o caso em que são reais. Na prova apresentamos o caso complexo e no seu último parágrafo mencionamos que o caso real é análogo utilizando um processo de complexificação de espaços de Banach. 


\section{Observação 147.}

Seja $X$ um espaço de Banach sobre o conjunto dos números reais. Definimos $\widehat{X}=$ $\left\{\left(x, x^{\prime}\right) \in X \times X: x, x^{\prime} \in X\right\}$ como o espaço complexificado de $X$. Mostraremos a seguir que com a multiplicação por escalar $(a+i b)\left(x, x^{\prime}\right)=\left(a x-b x^{\prime}, b x+a x^{\prime}\right)$ e a norma $\|(x, y)\|=\sup \left\{\sqrt{\left\|a x-b x^{\prime}\right\|^{2}+\left\|b x+a x^{\prime}\right\|^{2}}: a^{2}+b^{2}=1\right\}$ o espaço complexificado $\widehat{X}$ é um espaço de Banach. Com efeito, se $a=1$ e $b=0$ temos que $\|x\| \leq \sqrt{\|x\|^{2}+\left\|x^{\prime}\right\|^{2}} \leq$ $\left\|\left(x, x^{\prime}\right)\right\|, \forall\left(x, x^{\prime}\right) \in \widehat{X}$ e $\left\|x^{\prime}\right\| \leq\left\|\left(x, x^{\prime}\right)\right\|$. Então

$$
\max \left(\|x\|,\left\|x^{\prime}\right\|\right) \leq\left\|\left(x, x^{\prime}\right)\right\|, \forall\left(x, x^{\prime}\right) \in \widehat{X}
$$

Além disso, como $a^{2} \leq a^{2}+b^{2} \leq 1$ e $b^{2} \leq a^{2}+b^{2} \leq 1$ então

$$
\begin{aligned}
\left\|a x-b x^{\prime}\right\|^{2} \leq & \left(|a|\|x\|+|b|\left\|x^{\prime}\right\|\right)^{2}=a^{2}\|x\|^{2}+b^{2}\left\|x^{\prime}\right\|^{2}+2\left|a\|b \mid\| x\|\| x^{\prime} \| \leq\right. \\
& \leq\|x\|^{2}+\left\|x^{\prime}\right\|^{2}+2\|x\|\left\|x^{\prime}\right\|=\left(\|x\|+\left\|x^{\prime}\right\|\right)^{2}
\end{aligned}
$$

e $\left\|b x+a x^{\prime}\right\|^{2} \leq\left(\|x\|+\left\|x^{\prime}\right\|\right)^{2}$, implicando que $\sqrt{\left\|a x-b x^{\prime}\right\|^{2}+\left\|b x+a x^{\prime}\right\|^{2}} \leq \sqrt{2}(\|x\|+$ $\left.\left\|x^{\prime}\right\|\right)$ e então

$$
\left\|\left(x, x^{\prime}\right)\right\| \leq \sqrt{2}\left(\|x\|+\left\|x^{\prime}\right\|\right) .
$$

A desigualdade (4.14) implica que toda seqüência de Cauchy em $\widehat{X}$ converge em $\widehat{X}$ e portanto $\widehat{X}$ é um espaço de Banach.

Dado um operador real $T \in L(X, Y)$ então ele induz um operador complexo $\widehat{T}: \widehat{X} \rightarrow \widehat{Y}$ definido por $\widehat{T}\left(x, x^{\prime}\right)=\left(T(x), T\left(x^{\prime}\right)\right), \forall\left(x, x^{\prime}\right) \in \widehat{X}$. Obviamente $\widehat{T}$ é linear. Segue da continuidade de $T$ e de (4.15) que $\widehat{T}$ é contínuo. Se $P \in L(X, Y)$ é uma projeção real então o operador complexo $\widehat{P}: \widehat{X} \rightarrow \widehat{Y}$ induzido por $P$ também é uma projeção. De fato, dado $\left(x, x^{\prime}\right) \in \widehat{X}$ temos que

$$
\begin{gathered}
\widehat{P}^{2}\left(x, x^{\prime}\right)=\widehat{P} \widehat{P}\left(x, x^{\prime}\right)=\widehat{P}\left(P(x), P\left(x^{\prime}\right)\right)= \\
=\left(P^{2}(x), P^{2}\left(x^{\prime}\right)\right)=\widehat{P}\left(x, x^{\prime}\right) .
\end{gathered}
$$

Se $\widehat{T}_{1}: \widehat{X} \rightarrow \widehat{X}$ e $\widehat{T}_{2}: \widehat{X} \rightarrow \widehat{X}$ são operadores induzidos por $T_{1}: X \rightarrow X$ e $T_{2}: X \rightarrow X$, respectivamente, então $\widehat{T}_{1}+\widehat{T}_{2}=\widehat{T}_{1+T_{2}}$. De fato, dado $\left(x, x^{\prime}\right) \in \widehat{X}$ temos que

$$
\left(\widehat{T}_{1}+\widehat{T}_{2}\right)\left(x, x^{\prime}\right)=\left(\left(T_{1}+T_{2}\right)(x),\left(T_{1}+T_{2}\right)\left(x^{\prime}\right)\right)=\left(\widehat{T}_{1}+T_{2}\right)\left(x, x^{\prime}\right) .
$$


Se $\widehat{T}: \widehat{X} \rightarrow \widehat{Y}$ é o operador induzido pelo operador real $T: X \rightarrow Y$ e $\widehat{Q}: \widehat{X} \rightarrow \widehat{Y}$ é o operador induzido pelo operador real $Q: X \rightarrow Y$ então $\widehat{Q T}=\widehat{Q} \widehat{T}$. Com efeito, dado $\left(x, x^{\prime}\right) \in \widehat{X}$ temos que

$$
\widehat{Q} \widehat{T}\left(x, x^{\prime}\right)=\widehat{Q}\left(T(x), T\left(x^{\prime}\right)\right)=\left(Q T(x), Q T\left(x^{\prime}\right)\right)=\widehat{Q T}\left(x, x^{\prime}\right) .
$$

Se $T: X \rightarrow X$ é um operador real com inversa contínua $T^{-1}: X \rightarrow X$ então é fácil ver que o operador $\widehat{T}: \widehat{X} \rightarrow \widehat{X}$ induzido por $T$ tem inversa contínua $(\widehat{T})^{-1}=\widehat{T^{-1}}$.

Se $T_{n}: X \rightarrow X$ é uma seqüência de operadores reais que converge na norma de $L(X)$ para um operador real $T$ então $\widehat{T_{n}} \rightarrow \widehat{T}$. De fato, dado $\left(x, x^{\prime}\right) \in \widehat{X}$ temos que

$$
\begin{gathered}
\left\|\widehat{T_{n}}\left(x, x^{\prime}\right)-\widehat{T}\left(x, x^{\prime}\right)\right\|=\left\|\left(\left(T_{n}-T\right)(x),\left(T_{n}-T\right)\left(x^{\prime}\right)\right)\right\| \leq \\
\leq \sqrt{2}\left(\left\|\left(T_{n}-T\right)(x)\right\|+\left(T_{n}-T\right)\left(x^{\prime}\right) \|\right)
\end{gathered}
$$

que converge a zero quando $n \rightarrow \infty$.

A classe de todos os operadores induzidos por operadores reais é fechada, isto é, se $\left\{\widehat{T_{n}}\right\}$ é uma seqüência de operadores induzidos por operadores reais de $X$ em $Y$, onde $X$ e $Y$ são espaços de Banach sobre o conjunto dos números reais, tal que $T_{n} \rightarrow L$ então existe $T: X \rightarrow Y$ tal que $L=\widehat{T}$. De fato, dado $\left(x, x^{\prime}\right) \in \widehat{X}$, desde que $T_{n} \rightarrow L$, quando $n \rightarrow \infty$, então temos que $L\left(x, x^{\prime}\right)=\left(\lim _{n \rightarrow \infty} T_{n}(x), \lim _{n \rightarrow \infty} T_{n}\left(x^{\prime}\right)\right)$, que é um operador linear devido a linearidade de $T_{n}$ e do limite e também é contínuo, devido a continuidade de $T_{n}$ e do limite. Definindo $T: X \rightarrow Y$ por $T(x)=\lim _{n \rightarrow \infty} T_{n}(x), \forall x \in X$, temos que $L=\widehat{T}$.

Lema 148. Seja $Z$ um espaço de Banach real e $Q \in L(Z)$. Seja $\Gamma$ o bordo de um retângulo simétrico ao eixo real sobre um plano complexo e que não intercepta $\sigma(\widehat{Q})$. Então a projeção $\frac{1}{2 \pi i} \int_{\Gamma} R_{\widehat{Q}}(\lambda) d \lambda$ em $\widehat{Z}$ é induzido por alguma projeção real em $Z$.

Prova. Como $\frac{1}{2 \pi i} \int_{\Gamma} R_{\widehat{Q}}(\lambda) d \lambda$ é soma de Riemman então basta mostrarmos que esta soma é um operador induzido por um operador real. Para fazer isto tomamos uma partição do bordo de $\Gamma$ contendo os vértices e com os pontos dessa partição simétricos com relação ao eixo real e ao eixo imaginário. Então essa soma será da forma

$$
\frac{1}{2 \pi i} \sum\left(a_{k} R_{\widehat{Q}}\left(\lambda_{k}\right)-\overline{a_{k}} R_{\widehat{Q}}\left(\overline{\lambda_{k}}\right)\right)
$$


onde $a_{k}$ são reais ou imaginários puros. Se $a_{k}$ é real então

$$
\begin{aligned}
\left(a_{k} R_{\widehat{Q}}\left(\lambda_{k}\right)-a_{k} R_{\widehat{Q}}\left(\overline{\lambda_{k}}\right)\right) & =a_{k}\left(\left(\lambda_{k} I-\widehat{Q}\right)^{-1}-\left(\overline{\lambda_{k}} I-\widehat{Q}\right)^{-1}\right)= \\
& =a_{k}\left(\left(\lambda_{k}-\overline{\lambda_{k}}\right)\left(\lambda_{k} I-\widehat{Q}\right)^{-1}\left(\overline{\lambda_{k}} I-\widehat{Q}\right)^{-1}=\right. \\
& =a_{k}\left(\lambda_{k}-\overline{\lambda_{k}}\right)\left(\widehat{Q}^{2}+\left|\lambda_{k}\right|^{2} I-\left(\lambda_{k}+\overline{\lambda_{k}}\right) \widehat{Q}\right)^{-1}
\end{aligned}
$$

Se $a_{k}$ é imaginário puro então

$$
\begin{aligned}
\left(a_{k} R_{\widehat{Q}}\left(\lambda_{k}\right)-\overline{a_{k}} R_{\widehat{Q}}\left(\overline{\lambda_{k}}\right)\right) & \left.=a_{k} R_{\widehat{Q}}\left(\lambda_{k}\right)-\left(-a_{k}\right) R_{\widehat{Q}}\left(\overline{\lambda_{k}}\right)\right)= \\
& =a_{k}\left(R_{\widehat{Q}}\left(\lambda_{k}\right)+R_{\widehat{Q}}\left(\overline{\lambda_{k}}\right)\right)= \\
& =a_{k}\left(\lambda_{k}+\overline{\lambda_{k}}\right)\left(\widehat{Q}^{2}+\left|\lambda_{k}\right|^{2} I-\left(\lambda_{k}+\overline{\lambda_{k}}\right) \widehat{Q}\right)^{-1}
\end{aligned}
$$

Em ambos os casos obtemos somandos que consistem de um escalar imaginário puro multiplicado por um operador induzido de um operador real. Então a soma (4.16) é um operador real e como a classe dos operadores induzidos é fechada então também a integral $\frac{1}{2 \pi i} \int_{\Gamma} R_{\widehat{Q}}(\lambda) d \lambda$ é uma projeção induzida por uma projeção real.

Teorema 149. Sejam $X$ e $Y$ dois espaços de Banach tais que todo operador de $Y$ em $X$ é estritamente singular. Seja $P$ uma projeção de $X \oplus Y$ sobre um subespaço de dimensão infinita $Z$. Então existe um isomorfismo $\tau_{\circ}$ de $X \oplus Y$ em $X \oplus Y$ e subespaços complementados $X_{\mathrm{o}}$ de $X$ e $Y_{\circ}$ de $Y$ tais que $\tau_{\circ}(Z)=X_{\circ} \oplus Y_{\circ}$.

Prova. Inicialmente assumiremos que $X$ e $Y$ são espaços de Banach sobre o conjunto dos números complexos. Consideraremos os seguintes passos:

$1^{\underline{0}}$ ) A decomposição natural da projeção $P$ em operadores de $X$ em $X$, de $X$ em $Y$, de $Y$ em $X$ e de $Y$ em $Y$ e escreveremos $P$ em sua representação matricial em termos destes operadores.

$2^{\underline{Q}}$ ) Obteremos a partir de $P$ um operador $Q \in L(X \oplus Y)$ tal que $P-Q$ é estritamente singular e cujo espectro é enumerável com 0 e 1 sendo seus únicos pontos de acumulação.

$3^{\underline{Q}}$ ) Consideraremos então uma outra projeção $\widetilde{P}$ sobre $X \oplus Y$.

$4^{\underline{O}}$ ) Obteremos a partir de $\widetilde{P}$ um operador de Fredholm $T_{1}$ em $X \oplus Y$ de índice 0.

$5^{\mathrm{O}}$ ) Construiremos um isomorfismo $T_{2}$ em $X \oplus Y$. 
$6^{\underline{0}}$ ) A partir deste isomorfismo obteremos um operador de Fredholm $T \in L(X \oplus Y)$ de índice 0 que permite considerar um isomorfismo $\tau_{1}$ de $X \oplus Y$ em $X \oplus Y$. Então é considerado um isomorfismo $\tau_{2}$ em um subespaço de $X \oplus Y$ que leva $\tau_{1}(Z)$ sobre a soma direta de subespaços $X_{o}$ complementado em $X$ com $Y_{o}$ complementado em $Y$.

$7^{\underline{0}}$ ) Consideraremos então sua extensão $\widehat{\tau_{2}}$ ao espaço $X \oplus Y$.

$8^{\text {O }}$ ) Finalmente obteremos o isomorfismo $\tau_{0}$ desejado.

10 passo.) Escrevemos a projeção $P: X \oplus Y \rightarrow Z \subset X \oplus Y$ do seguinte modo

$$
P(x, y)=\left(T_{1}(x, y), T_{2}(x, y)\right), \forall(x, y) \in X \oplus Y,
$$

onde $T_{1}: X \oplus Y \rightarrow X$ e $T_{2}: X \oplus Y \rightarrow Y$ são operadores lineares. Temos que

$$
P(x, y)=\left(T_{1}(x, y), T_{2}(x, y)\right)=\left(T_{1}\left(\pi_{1}(x)\right)+T_{1}\left(\pi_{2}(y)\right), T_{2}\left(\pi_{1}(x)\right)+T_{2}\left(\pi_{2}(y)\right)\right),
$$

onde $\pi_{1}: X \rightarrow X \oplus\{0\}$ e $\pi_{2}: Y \rightarrow\{0\} \oplus Y$ são operadores lineares definidos, respectivamente, por $\pi_{1}(x)=(x, 0), \forall x \in X$ e $\pi_{2}(y)=(0, y), \forall y \in Y$. Logo,

$$
P(x, y)=\left(S_{1}(x)+S_{3}(y), S_{2}(x)+S_{4}(y)\right), \forall(x, y) \in X \oplus Y,
$$

onde $S_{1}=T_{1} \pi_{1}: X \rightarrow X, S_{2}=T_{2} \pi_{1}: X \rightarrow Y, S_{3}=T_{1} \pi_{2}: Y \rightarrow X$ e $S_{4}=T_{2} \pi_{2}: Y \rightarrow Y$, ou ainda, na representação matricial

$$
P=\left(\begin{array}{cc}
S_{1} & S_{3} \\
S_{2} & S_{4}
\end{array}\right)
$$

20 passo.) Pondo

$$
Q=\left(\begin{array}{cc}
S_{1} & 0 \\
S_{2} & S_{4}
\end{array}\right)
$$

ou ainda, $Q(x, y)=\left(S_{1}(x), S_{2}(x)+S_{4}(y)\right), \forall(x, y) \in X \oplus Y$, temos que

$$
P-Q=\left(\begin{array}{cc}
0 & S_{3} \\
0 & 0
\end{array}\right) \in S S(X \oplus Y),
$$

pois $S_{3} \in S S(Y, X)$. Substituiremos $Q$ por uma projeção $\widetilde{P}$ em $X \oplus Y$ que possua uma representação matricial similar à de $Q$.

3 o passo.) Consideremos o operador linear

$$
\widetilde{P}=\frac{1}{2 \pi i} \int_{\Gamma} R_{Q}(\lambda) d \lambda,
$$


onde $R_{Q}(\lambda)$ é o operador resolvente de $Q$ e $\Gamma \subset \mathbb{C}$ é uma curva simples, fechada, que não intercepta o espectro $\sigma(Q)$ de $Q$, possui o número 1 em seu interior e os números 0 e 2 em seu exterior. Tal curva existe pelo Lema 145.

O operador $\widetilde{P}$ é uma projeção em $X \oplus Y$ pois aplicando a Proposição 137(ii) à função analítica $g: \mathbb{C} \rightarrow \mathbb{C}$ definida por $g(\lambda)=1, \forall \lambda \in \mathbb{C}$ e sendo $\widetilde{P}=g(Q)$ (ver (3.22)) temos que

$$
\widetilde{P} \circ \widetilde{P}=g(Q) \circ g(Q)=(g \circ g)(Q)=g(Q)=\widetilde{P}
$$

Temos também que $\widetilde{P}$ comuta com $Q$. De fato, segue da Observação 136, fórmula (3.22) para $k=1$, que

$$
Q=\frac{1}{2 \pi i} \int_{\Gamma} \lambda R_{Q}(\lambda) d \lambda
$$

e é o operador associado à função analítica $f: \mathbb{C} \rightarrow \mathbb{C}$ definida por $f(\lambda)=\lambda, \forall \lambda \in \mathbb{C}$. Temos que $f \circ g=g \circ f$ pois

$$
\begin{aligned}
& (f \circ g)(\lambda)=f(g(\lambda))=f(1)=1 \\
& (g \circ f)(\lambda)=g(f(\lambda))=g(\lambda)=1 .
\end{aligned}
$$

Segue da Proposição 137-(ii) que $Q \circ \widetilde{P}=f(Q) \circ g(Q)=(f \circ g)(Q)=g(Q)=\widetilde{P}$, implicando que $Q \widetilde{P}(X \oplus Y) \subset \widetilde{P}(X \oplus Y)$.

Denotemos por $I$ o operador identidade sobre $X \oplus Y$. Seja $\alpha \in \operatorname{ext}(\Gamma)$. Temos que o operador $\alpha I-Q \widetilde{P}_{\widetilde{P}_{(X \oplus Y)}}=\alpha I-\widetilde{P}$ é inversível pois segue da Proposição 138 que

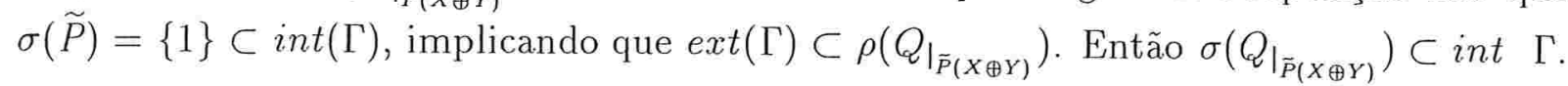
Pelo mesmo argumento temos que $\sigma\left(Q_{\left.\right|_{(I-\bar{P})(X \oplus Y)}}\right) \subset \operatorname{ext}(\Gamma)$, pois dado $\alpha \in \operatorname{int}(\Gamma)$ segue que $\alpha I-Q(I-\widetilde{P})=\alpha I-(I-P)$, que é inversível, pois $\sigma(I-P)=\{0\} \subset \operatorname{ext}(\Gamma)$. Deste modo, int $(\Gamma) \subset \rho\left(Q_{\left.\right|_{(I-\widetilde{P})(X \oplus Y)}}\right)$ implicando que $\sigma\left(Q_{\left.\right|_{(I-\widetilde{P})(X \oplus Y)}}\right) \subset \operatorname{ext}(\Gamma)$. Além disso, podemos escrever

$$
\widetilde{P}=\left(\begin{array}{ll}
P_{1} & 0 \\
P_{2} & P_{4}
\end{array}\right),
$$

onde $P_{1}$ é uma projeção em $X, P_{4}$ é uma projeção em $Y$ e $P_{2}$ é um operador linear de $X$ em $Y$.

4 o passo.) O operador $I+\widetilde{P}-Q$ sobre $X \oplus Y$ deixa $\widetilde{P}(X \oplus Y)$ invariante e é inversivel nesse subespaço. Com efeito, dado $(x, y) \in X \oplus Y$ temos que

$$
\begin{aligned}
& (I+\widetilde{P}-Q) \widetilde{P}(x, y)=\widetilde{P}(x, y)+\widetilde{P}^{2}(x, y)-Q \widetilde{P}(x, y)= \\
= & 2 \widetilde{P}(x, y)-Q \widetilde{P}(x, y)=2 \widetilde{P}(x, y)-\widetilde{P} Q(x, y) \in \widetilde{P}(X \oplus Y) .
\end{aligned}
$$


Além disso, como $(I+\widetilde{P}-Q)_{\left.\right|_{\tilde{P}(X \oplus Y)}}=(2 I-Q)_{\left.\right|_{\tilde{P}(X \oplus Y)}}, 2 \in \operatorname{ext}(\Gamma)$ e $\sigma\left(Q_{\left.\right|_{\Gamma_{P}(X \nRightarrow Y)}}\right) \subset$ $\operatorname{int}(\Gamma)$ então segue que $2 \in \rho(Q)$ e portanto $(2 I-Q)_{\left.\right|_{\vec{P}(X \oplus Y)}}$ é inversível sobre $\widetilde{P}(X \oplus Y)$.

O operador $I+\widetilde{P}-Q$ sobre $X \oplus Y$ também deixa $(I-\widetilde{P})(X \oplus Y)$ invariante e é inversível sobre este subespaço. De fato, dado $(x, y) \in X \oplus Y$ temos que

$$
\begin{gathered}
(I+\widetilde{P}-Q)(I-\widetilde{P})(x, y)=(I+\widetilde{P}-Q)(x, y)-(I+\widetilde{P}-Q) \widetilde{P}(x, y)= \\
=I(x, y)-Q(x, y)-\widetilde{P}(x, y)+Q \widetilde{P}(x, y)=(I-\widetilde{P})(x, y)-Q(I-\widetilde{P})(x, y)= \\
=(I-\widetilde{P})(x, y)-(I-\widetilde{P}) Q(x, y) \in(I-\widetilde{P})(X \oplus Y) .
\end{gathered}
$$

Além disso, como $(I+\widetilde{P}-Q)_{\left.\right|_{(I-\widetilde{P})(X \oplus Y)}}=(I-Q)_{\left.\right|_{(I-\tilde{P})(X \oplus Y)}}, 1 \in \operatorname{int}(\Gamma)$ e $\sigma\left(Q_{\mathrm{I}_{(I-\widetilde{P})(X \oplus Y)}}\right) \subset$ $\operatorname{ext}(\Gamma)$ então segue que $I+\widetilde{P}-Q$ é inversivel sobre $(I-\widetilde{P})(X \oplus Y)$.

Segue pelo Lema 142 que o operador $I+\widetilde{P}-Q$ é inversível sobre $X \oplus Y$. Deste modo, segue da Proposição 104 que

$$
T_{1}=I+\widetilde{P}-P=(I+\widetilde{P}-Q)+(Q-P)
$$

é um operador de Fredholm de índice 0 , pois $Q-P \in S S(X \oplus Y)$ e $i(I+\widetilde{P}-Q)=0$.

Mostraremos agora que o subespaço $T_{1}(Z)$ é de codimensão finita em $\widetilde{P}(X \oplus Y)$. De fato, dado $(x, y) \in X \oplus Y$ temos que

$$
\left(T_{1} P\right)(x, y)=(I+\widetilde{P}-P) P(x, y)=P(x, y)+\widetilde{P} P(x, y)-P^{2}(x, y)=\widetilde{P} P(x, y),
$$

implicando que $T_{1} P=\widetilde{P} P$. Então $T_{1}(Z)=T_{1} P(X \oplus Y) \subset \widetilde{P}(X \oplus Y)$.

Para mostrar que $T_{1}(Z)$ é de codimensão finita em $\widetilde{P}(X \oplus Y)$ basta mostrar que $Z$ é de codimensão finita em $T_{1}^{-1}(\widetilde{P}(X \oplus Y))$. Se $\operatorname{dim}\left(\frac{T_{1}^{-1}(\widetilde{P}(X \oplus Y))}{Z}\right)=\infty$ então existe um subespaço de dimensão infinita $W$ de $X \oplus Y$ tal que $P_{\left.\right|_{W}}=0, T_{\left.1\right|_{W}}$ é um isomorfismo e $T_{1}(W) \subset \widetilde{P}(X \oplus Y)$. Para $w \in W$ temos que $T_{1}(w)=(I+\widetilde{P}-P)(w)=w+\widetilde{P}(w)-P(w)=$ $w+\widetilde{P}(w)$. Logo, $w=T_{1}(w)-\widetilde{P}(w) \in \widetilde{P}(X \oplus Y)$, ou seja, $W \subset \widetilde{P}(X \oplus Y)$. Mas, sobre $\widetilde{P}(X \oplus Y)$ o operador $Q=0 I-(-Q)$ é inversível $\left(\right.$ pois $\sigma\left(Q_{\left.\right|_{\tilde{P}(X \oplus Y)}}\right) \subset \operatorname{int}(\Gamma)$ e $\left.0 \in \operatorname{ext}(\Gamma)\right)$ e $Q_{\left.\right|_{W}}=(Q-P)_{\left.\right|_{W}}$, que é uma contradição, pois $Q-P \in S S(X \oplus Y)$.

5으 passo.) Consideremos a projeção $B: X \oplus Y \rightarrow X \oplus Y$ definida por $B(x, y)=$ $\left(P_{1}(x), P_{4}(y)\right)$. O próximo passo na prova é construir um isomorfismo $T_{2}: X \oplus Y \rightarrow X \oplus Y$ de modo que $T_{2} \widetilde{P} T_{2}^{-1}=B$. Seja $S=\widetilde{P}-B$. Observemos que

$$
S^{2}=S \widetilde{P} S=\widetilde{P} S \widetilde{P}=0
$$

Definindo o operador $\widetilde{S}=S \widetilde{P}-\widetilde{P} S: X \oplus Y \rightarrow X \oplus Y$ temos que 


$$
\begin{gathered}
\widetilde{S}^{2}=\widetilde{S} \widetilde{S}=\widetilde{S}(S \widetilde{P}-\widetilde{P} S)=(S \widetilde{P}-\widetilde{P} S)(S \widetilde{P}-\widetilde{P} S)= \\
=S \widetilde{P} S \widetilde{P}-S \widetilde{P} S-\widetilde{P} S^{2} \widetilde{P}+\widetilde{P} S \widetilde{P} S=0 .
\end{gathered}
$$

O operador $T_{2}=I-\widetilde{S}: X \oplus Y \rightarrow X \oplus Y$ é um isomorfismo cuja inversa é o operador $T_{2}^{-1}=I+\widetilde{S}: X \oplus Y \rightarrow X \oplus Y$, pois

$$
T_{2} T_{2}^{-1}=(I-\widetilde{S})(I+\widetilde{S})=I-\widetilde{S}+\widetilde{S}-\widetilde{S}^{2}=I=T_{2}^{-1} T_{2} .
$$

Observemos ainda que

$$
\begin{gathered}
(I-\widetilde{S}) \widetilde{P}(I+\widetilde{S})=(I-S \widetilde{P}+\widetilde{P} S) \widetilde{P}(I+S \widetilde{P}-\widetilde{P} S)= \\
=(I-S \widetilde{P}+\widetilde{P} S)(\widetilde{P}+\widetilde{P} S \widetilde{P}-\widetilde{P} S)=(I-S \widetilde{P}+\widetilde{P} S) \widetilde{P}-(I-S \widetilde{P}+\widetilde{P} S) \widetilde{P} S= \\
=\widetilde{P}-S \widetilde{P}+\widetilde{P} S \widetilde{P}-\widetilde{P} S+S \widetilde{P} S+\widetilde{P} S \widetilde{P} S=\widetilde{P}-S \widetilde{P}-\widetilde{P} S .
\end{gathered}
$$

Por outro lado,

$$
\begin{aligned}
(\widetilde{P}-S) \widetilde{P}(\widetilde{P}-S)= & (\widetilde{P}-S)(\widetilde{P}-\widetilde{P} S)=(\widetilde{P}-S) \widetilde{P}-(\widetilde{P}-S) \widetilde{P} S= \\
& =\widetilde{P}-S \widetilde{P}-\widetilde{P} S+S \widetilde{P} S
\end{aligned}
$$

Então

$$
T_{2} \widetilde{P} T_{2}^{-1}=(I-\widetilde{S}) \widetilde{P}(I+\widetilde{S})=(\widetilde{P}-S) \widetilde{P}(\widetilde{P}-S)=B \widetilde{P} B=B
$$

$6^{0}$ o passo.) Como $T_{2}$ é inversível sobre $X \oplus Y$ segue que $i\left(T_{2}\right)=i\left(T_{2}^{-1}\right)=0$, implicando que $T=T_{2} T_{1} T_{2}^{-1}$ é um operador de Fredholm e

$$
i(T)=i\left(T_{2} T_{1} T_{2}^{-1}\right)=i\left(T_{2}\right)+i\left(T_{1}\right)+i\left(T_{2}^{-1}\right)=0 .
$$

Além disso, $T(Z)$ é um subespaço de codimensão finita em $R(B)=X_{1} \oplus Y_{1}$, onde $X_{1}=P_{1}(X)$ e $Y_{1}=P_{4}(Y)$.

Como $T$ é um operador de Fredholm de índice 0 então pelo Lema 141 existe um operador de posto finito $H: X \oplus Y \rightarrow X \oplus Y$ tal que o operador $\tau_{1}=T+H$ é um isomorfismo de $X \oplus Y$ em $X \oplus Y$.

Temos que $\tau_{1}(Z)=T(Z) \oplus H(Z)$. Como $T(Z)$ é de codimensão finita em $X_{1} \oplus Y_{1}$, então existe um subespaço de dimensão finita $D$ de $X_{1} \oplus Y_{1}$ tal que

$$
X_{1} \oplus Y_{1}=D \oplus T(Z),
$$

pois como $Z$ é complementado (imagem da projeção $P$ ) então é fechado e a imagem de um subespaço fechado por um operador de Fredholm é também um subespaço fechado. Por (4.17) temos que 
$X_{1} \oplus Y_{1} \oplus H(Z)=D \oplus T(Z) \oplus H(Z)$.

Colocando $C=H(Z)$ obtemos que

$$
\tau_{1}(Z) \oplus D=X_{1} \oplus Y_{1} \oplus C
$$

onde $\operatorname{dim}(C)<\infty$. Pelo Lema 139 podemos escrever $C=C_{X} \oplus C_{Y}$, onde $C_{X} \subset X, C_{Y} \subset$ $Y, \operatorname{dim}\left(C_{X}\right)<\infty$ e $\operatorname{dim}\left(C_{Y}\right)<\infty$. Então segue de (4.18) que

$\tau_{1}(Z) \subset \tau_{1}(Z) \oplus D=X_{1} \oplus Y_{1} \oplus C=X_{1} \oplus Y_{1} \oplus C_{X} \oplus C_{Y}=\left(X_{1} \oplus C_{X}\right) \oplus\left(Y_{1} \oplus C_{Y}\right)$.

Deste modo, $\tau_{1}(Z) \subset X_{2} \oplus Y_{2}$, onde $X_{2}=X_{1} \oplus C_{X}$ e $Y_{2}=Y_{1} \oplus C_{Y}$.

Pelo Lema 140 temos que se $X_{o}$ e $Y_{o}$ são subespaços de codimensão finita em $X_{2}$ e $Y_{2}$, respectivamente, tais que

$$
\operatorname{dim}\left(\frac{X_{2} \oplus Y_{2}}{\tau_{1}(Z)}\right)=\operatorname{dim}\left(\frac{X_{2} \oplus Y_{2}}{X_{o} \oplus Y_{o}}\right)<\infty
$$

então existe um isomorfismo sobrejetor $\tau_{2}: X_{2} \oplus Y_{2} \rightarrow X_{2} \oplus Y_{2}$ que leva $\tau_{1}(Z)$ sobre $X_{o} \oplus Y_{o}$. Temos que $X_{2} \oplus Y_{2}$ é complementado em $X \oplus Y$, isto é,

$$
X \oplus Y=X_{2} \oplus Y_{2} \oplus E,
$$

onde $E$ é um subespaço fechado de $X \oplus Y$.

$7 \underline{\mathbf{o}}$ pasșo.) Então $\tau_{2}$ pode ser extendido a um isomorfismo $\hat{\tau_{2}}$ de $X \oplus Y$ definido por

$$
\hat{\tau_{2}}(u+e)=\tau_{2}(u)+e,
$$

onde $e \in E$ e $u \in X_{2} \oplus Y_{2}$.

8o passo.) Conseqüentemente, $\tau_{o}=\hat{\tau}_{2} \tau_{1}: X \oplus Y \rightarrow X \oplus Y$ é um isomorfismo tal que $\tau_{o}(Z)=X_{o} \oplus Y_{o}$. Como $X_{o}$ é complementado em $X$ e $Y_{o}$ é complementado em $Y$ então o resultado está provado para o caso complexo.

9ọ passo.) O caso real é provado de modo análogo utilizando um processo de complexificação de espaços de Banach conforme apresentado na Observação 147. Se $X$ e $Y$ são espaços de Banach então definimos os espaços $\widehat{X}=\{(u, v): u, v \in X\} \operatorname{com}(a+b i)(u, v)=$ $(a u-b v, a v+b u)$ e com a norma $\|(u, v)\|=\max \left\{\left(\|a u-b v\|^{2}+\|a v+b u\|^{2}\right)^{\frac{1}{2}}: a^{2}+b^{2}=1\right\}$ e $\widehat{Y}=\{(c, d): c, d \in Y\}$. A projeção $P$ sobre $X \oplus Y$ induz uma projeção $\widehat{P}$ sobre $\widehat{X} \oplus \widehat{Y}$. Tomando $\Gamma$ como sendo um retângulo simétrico com respeito ao eixo real asseguramos que o operador $\widetilde{P}=\frac{1}{2 \pi i} \int_{\Gamma} R_{Q}(\lambda) d \lambda$ utilizado na demonstração é da forma $\widehat{\widetilde{P}}$ para o operador $\widetilde{P}$. A prova então é análoga ao caso complexo. 
Observação 150.

Vimos no Exemplo 40 que $l_{1}(\mathbb{N})$ e $l_{2}(\mathbb{N})$ são totalmente incomparáveis e portanto $L\left(l_{1}(\mathbb{N}), l_{2}(\mathbb{N})\right)=S S\left(l_{1}(\mathbb{N}), l_{2}(\mathbb{N})\right)$. Então, pelo teorema anterior, qualquer subespaço complementado de dimensão infinita $M$ em $l_{1}(\mathbb{N}) \oplus l_{2}(\mathbb{N})$ é isomorfo à soma $X_{o} \oplus Y_{o}$, onde $X_{o}$ é um subespaço complementado de $l_{1}(\mathbb{N})$ e $Y_{o}$ é um subespaço complementado de $l_{2}(\mathbb{N})$. Como todo subespaço complementado de dimensão infinita em $l_{p}(\mathbb{N}), 1 \leq p \leq \infty$, é isomorfo a $l_{p}(\mathbb{N})([22])$ então temos que $M$ é isomorfo a $l_{1}(\mathbb{N})$ ou a $l_{2}(\mathbb{N})$ ou a $l_{1}(\mathbb{N}) \oplus l_{2}(\mathbb{N})$. 



\section{Operadores inessenciais e improjetivos}

Os operadores inessenciais foram introduzidos por Kleinecke, D., em 1963, em seu estudo da teoria de perturbação de operadores de Fredholm. Inicialmente essa classe de operadores foi definida para operadores atuando sobre um mesmo espaço de Banach. Outros autores [24] generalizaram o conceito de operadores inessenciais para operadores atuando entre espaços de Banach distintos. Nesse capítulo observaremos que a classe dos operadores inessenciais constitui um ideal de operadores (veja Observação 152). Apresentaremos alguns resultados de simetria desta classe e algumas caracterizações dos operadores inessenciais (veja Proposição 165). Tarafdar, E. [29] e [30] introduziu o conceito de operadores improjetivos. Neste mesmo trabalho o autor mostrou que todo operador inessencial atuando entre o mesmo espaço de Banach é um operador improjetivo. González, M. e Aiena, P. [3] mostraram que este resultado ocorre também para operadores atuando entre espaços de Banach distintos (veja Proposição 181). Exibiremos nesse capítulo algumas caracterizações dos operadores improjetivos e alguns resultados mostrando casos particulares em que ocorre a igualdade $\operatorname{Im} p=\operatorname{In}$. Finalmente apresentaremos os contraexemplos dados por González, M. e Aiena, P. (veja. Teorema 195) que mostram que tal igualdade não ocorre em geral. 


\section{$5.1 \quad$ Operadores inessenciais}

Definição 151. Sejam $X$ e $Y$ espaços de Banach. Um operador $T: X \rightarrow Y$ é dito inessencial se o operador $I_{X}-S T \in \Phi(X)$ para todo operador $S \in L(Y, X)$. Denotaremos por In $(X, Y)$ a classe de todos os operadores inessenciais de $X$ em $Y$ e por In a classe de todos os operadores inessenciais entre quaisquer espaços de Banach.

\section{Observação 152.}

In é um ideal de operadores conforme vimos na Definição 95. Temos também que In é a classe de perturbação para a classe dos operadores de Fredholm $\Phi$, isto é, é o maior ideal de operadores verificando a seguinte propriedade

$$
\text { Se } T \in \Phi(X, Y) \text { e } K \in \operatorname{In}(X, Y) \text { então } T+K \in \Phi(X, Y) \text {. }
$$

Tal resultado segue da Proposição 116. Em particular, In contém as classes dos operadores compactos e estritamente singulares.

Definição 153. Dois espaços de Banach $Y$ e $Z$ são essencialmente incomparáveis se $L(Y, Z)=\operatorname{In}(Y, Z)$.

Exemplo 154. Se $Z=C[0,1]$ e $Y$ é um espaço de Banach que não contém cópias complementadas de $c_{o}(\mathbb{N})$ então $Y$ e $Z$ são essencialmente incomparáveis.

Notemos que qualquer cópia de $c_{o}(\mathbb{N})$ em $Z$ é um subespaço complementado. Deste modo, dado $S \in L(Y, Z)$ e $T \in L(Z, Y)$ se o operador $S T$ não é fracamente compacto então ele é urm isomorfismo em um subespaço $M$ isomorfo a $c_{o}(\mathbb{N})$ e $T(M)$ é um subespaço complementado isomorfo a $c_{0}(\mathbb{N})$ em $Y$.

Os conceitos a seguir servirão como subsídios para a verificação da caracterização dos operadores inessenciais devida a Pietsch, A. [23] que apresentaremos na Proposição 165. Também apresentaremos uma caracterização dual desta propriedade [1] (veja Proposição 170 dessa seção).

Definição 155. Sejam $X$ e $Y$ espaços de Banach e $T \in L(X, Y)$. Então o módulo de injeção de $T$ é definido por

$$
j(T)=\sup \{\tau \geq 0:\|T(x)\| \geq \tau\|x\|, \forall x \in X\} .
$$

Em particular, definimos $j(0)=0$. 
Definição 156. Sejam $X \in Y$ espaços de Banach. Um operador $T \in L(X, Y)$ é dito uma injeção se $j(T)>0$. Uma injeção pode ser caracterizada como um operador injetor de $X$ em $Y$ com imagem fechada.

Se $\|T\|=j(T)=1$ então $T$ é uma isometria. Isto significa que $\|T(x)\|=\|x\|, \forall x \in X$. Proposição 157. Sejam $X$ e $Y$ espaços de Banach, $T$ e $A \in L(X, Y)$. Então $j(T+A) \leq$ $J(T)+\|A\|$.

Prova. Temos que

$$
\|T(x)\| \geq\|T(x)+A(x)\|-\|A(x)\| \geq j(T+A)\|x\|-\|A\|\|x\| .
$$

Conseqüentemente, segue que $j(T+A) \leq j(T)+\|A\|$.

Proposição 158. Seja $X$ um espaço de Banach. Um subespaço $M$ de $X$ é complementado em $X$ se, e somente se, existem operadores $J \in L(M, X)$ e $Q \in L(X, M)$ tais que $I_{M}=$ $Q J$, onde $I_{M}$ é o operador identidade sobre $M$. Nesse caso $J$ é a inclusão canônica de $M$ em $X$.

Prova. Se $I_{M}=Q J$ então o operador $P=J Q$ é uma projeção com $M=R(P)$.

Reciprocamente, suponhamos que $M$ é um subespaço complementado em $X$. Então existe uma projeção $P \in L(X)$ tal que $M=R(P)$. Considerando $P$ como um operador linear de $X$ sobre $M$ obtemos um operador $Q \in L(X, M)$ com $I_{M}=Q J$, onde $J$ denota a inclusão canônica de $M \operatorname{em} X$.

Proposição 159. Seja $X$ um espaço de Banach. Um subespaço $N$ de $X$ é complementado em $X$ se, e somente se, existem operadores $J \in L(X / N, X)$ e $Q \in L(X, X / N)$ tais que $I_{X / N}=Q J$, onde $I_{X / N}$ é o operador identidade sobre $X / N$. Nesse caso $Q$ é a aplicação quociente canônica de $X$ sobre $X / N$.

Prova. É análoga a prova da proposição anterior.

Definição 160. Sejam $X$ e $Y$ espaços de Banach. Um operador $T \in L(X, Y)$ é dito uma $\Phi$-injeção se existe um subespaço de codimensão finita $M$ de $X$ tal que $T J_{M}^{X}$ é uma injeção.

Proposição 161. Sejam $X$ e $Y$ espaços de Banach e $S \in S S(X, Y)$. Se $T \in L(X, Y)$ é uma $\Phi$-injeção então também o é o operador $S+T$. 
Prova. Temos que existe um subespaço de codimensão finita $M$ de $X$ tal que $T J_{M}^{X}$ é uma injeção. Suponhamos que $S+T$ não seja uma $\Phi$-injeção. Então a restrição de $S+T$ a qualquer subespaço de codimensão finita de $X$ não é uma injeção. Segue da Proposição 49 que existe um subespaço de dimensão infinita $M_{o}$ de $M$ com

$$
\left\|(S+T) J_{M_{o}}^{X}\right\|<j\left(T J_{M}^{X}\right)
$$

onde $j\left(T J_{M}^{X}\right)>0$ pois $T J_{M}^{X}$ é uma injeção. Seja $A=\left\{\tau \geq 0:\left\|T J_{M}^{X}(x)\right\| \geq \tau\|x\|, \forall x \in M\right\}$ e $B=\left\{\tau \geq 0:\left\|T J_{M_{o}}^{X}(x)\right\| \geq \tau\|x\|, \forall x \in M_{o}\right\}$. Para qualquer $\tau_{o} \in A$ temos que $\tau_{o} \in B$, implicando que $j\left(T J_{M_{o}}^{X}\right) \geq \tau_{o}$. Segue daí que que $j\left(T J_{M}^{X}\right) \leq j\left(T J_{M_{o}}^{X}\right)$. Então segue de (5.1) que

$$
\left\|(S+T) J_{M_{o}}^{X}\right\|<j\left(T J_{M}^{X}\right)<j\left(T J_{M_{o}}^{X}\right)
$$

Temos que

$$
\begin{gathered}
\left\|S J_{M_{o}}^{X}(x)\right\|=\left\|S J_{M_{o}}^{X}(x)+T J_{M_{o}}^{X}(x)-T J_{M_{o}}^{X}(x)\right\| \geq\left\|T J_{M_{o}}^{X}(x)\right\|-\left\|(S+T) J_{M_{o}}^{X}(x)\right\| \geq \\
\geq j\left(T J_{M_{o}}^{X}\right)\|x\|-\left\|(S+T) J_{M_{o}}^{X}\right\|\|x\|=\left(j\left(T J_{M_{o}}^{X}\right)-\left\|(S+T) J_{M_{o}}^{X}\right\|\right)\|x\| .
\end{gathered}
$$

Deste modo, segue de (5.2) que $j\left(S J_{M_{o}}^{X}\right) \geq j\left(T J_{M_{o}}^{X}\right)-\left\|(S+T) J_{M_{o}}^{X}\right\|>0$ e então $S J_{M_{o}}^{X}$ é uma injeção. Como $S \in S S(X, Y)$ então $M_{0}$ é de dimensão finita, que é uma contradição.

Lema 162. Sejam $X$ e $Y$ espaços de Banach e $T \in L(X, Y)$ uma injeção. Se $A \in L(X, Y)$ e $\|A\|<j(T)$ então $T+A$ é uma injeção. Além disso, $R(T) \in \operatorname{Cod}(Y)$ se, e somente se, $R(T+A) \in \operatorname{Cod}(Y)$.

Prova. Para $0 \leq \lambda \leq 1$ temos, pela Proposição 157 , que

$$
j(T)=j(T+\lambda A-\lambda A) \leq j(T+\lambda A)+|\lambda|\|A\| \leq J(T+\lambda A)+\|A\|,
$$

implicando que

$$
j(T+\lambda A) \geq j(T)-\|A\|, \quad 0 \leq \lambda \leq 1 .
$$

Por hipótese temos que $j(T)-\|A\|>0$. Então o operador $T+\lambda A$ é uma injeção para $0 \leq \lambda \leq 1$. Em particular o operador $T+A$ é uma injeção.

Suponhamos que um, e somente um, dos subespaços $R(T)$ e $R(T+A)$ possui codimensão finita em $Y$. Então da.do $\varepsilon>0$ podemos encontrar $\lambda_{o}, \lambda_{1} \in[0,1]$ satisfazendo $\left|\lambda_{1}-\lambda_{0}\right|<\varepsilon$ tais que 


$$
M_{o}=R\left(T+\lambda_{o} A\right) \in \operatorname{Cod}(Y) \text { e } M_{1}=R\left(T+\lambda_{1} A\right) \notin \operatorname{Cod}(Y) .
$$

Como $M_{1} \notin \operatorname{Cod}(Y)$ então $M_{1} \neq Y$. Segue pela Proposição 55 que existe $y_{1} \in B_{Y}$ tal que $\left\|m-y_{1}\right\| \geq \frac{1}{1+\varepsilon}, \forall m \in M_{1}$, onde $B_{Y}$ denota a bola unitária de $Y$. Denotemos por $y_{1}\left(M_{1}\right)$ a classe de equivalência $\left\{y_{1}+m_{1}: m_{1} \in M_{1}\right\}$, cuja norma é $\left\|y_{1}\left(M_{1}\right)\right\|=$ $\inf \left\{\left\|y_{1}-m_{1}\right\|: m_{1} \in M_{1}\right\}$. Temos que $\left[\left\{y_{1}\right\} \cup M_{1}\right] \neq Y$, pois $M_{1} \notin \operatorname{Cod}(Y)$. Segue da. Proposição 55 que existe $y_{2} \in B_{Y}$ tal que $\left\|m-y_{2}\right\| \geq \frac{1}{1+\varepsilon}, \forall m \in\left[\left\{y_{1}\right\} \cup M_{1}\right]$. Também existe $y_{3} \in B_{Y}$ tal que $\left\|m-y_{3}\right\| \geq \frac{1}{1+\varepsilon}, \forall m \in\left[\left\{y_{1}, y_{2}\right\} \cup M_{1}\right] \mathrm{e}$

$$
\left\|y_{3}\left(M_{1}\right)-y_{1}\left(M_{1}\right)\right\|=\inf \left(\left\|y_{3}-m\right\| \geq \frac{1}{1+\varepsilon}: m \in\left[\left\{y_{1}, y_{2}\right\} \cup M_{1}\right]\right) .
$$

Deste modo obtemos uma seqüência $y_{1}, y_{2}, y_{3}, \ldots \in B_{Y}$ tal que

$$
\left\|y_{i}\left(M_{1}\right)-y_{k}\left(M_{1}\right)\right\| \geq \frac{1}{1+\varepsilon}, \quad i \neq k
$$

Por outro lado, como $Y / M_{0}$ é de dimensão finita então temos que

$$
\left\|y_{i}\left(M_{o}\right)-y_{k}\left(M_{o}\right)\right\|<\varepsilon \text {, para algum } i \neq k \text {. }
$$

Seja $x \in X$ tal que $\left\|y_{i}-y_{k}-\left(T+\lambda_{o} A\right)(x)\right\|<\varepsilon$. Então por $(5.3)$ temos que

$$
\begin{gathered}
(j(T)-\|A\|)\|x\| \leq j\left(T+\lambda_{o} A\right)\|x\| \leq\left\|\left(T+\lambda_{o} A\right)(x)\right\|= \\
=\left\|y_{i}-y_{k}-\left(T+\lambda_{o} A\right)(x)+\left(y_{k}-y_{i}\right)\right\| \leq\left\|y_{i}-y_{k}-\left(T+\lambda_{o} A\right)(x)\right\|+\left\|y_{k}-y_{i}\right\| \leq \varepsilon+2 .
\end{gathered}
$$

implicando que

$$
\begin{gathered}
\frac{1}{1+\varepsilon} \leq\left\|y_{i}\left(M_{1}\right)-y_{k}\left(M_{1}\right)\right\| \leq\left\|y_{i}-y_{k}-\left(T+\lambda_{1} A\right)(x)\right\|= \\
=\left\|y_{i}-y_{k}-T(x)-\lambda_{1} A(x)-\lambda_{o} A(x)+\lambda_{o} A(x)\right\| \leq \\
\leq\left\|y_{i}-y_{k}-\left(T+\lambda_{o} A\right)(x)\right\|+\left|\lambda_{o}-\lambda_{1}\right|\|A(x)\| \leq \varepsilon+\varepsilon\|A\|\|x\|= \\
=\varepsilon(1+\|A\|\|x\|) \leq \varepsilon\left[1+\frac{(2+\varepsilon)\|A\|}{j(T)-\|A\|}\right]
\end{gathered}
$$

que é falso para $\varepsilon$ suficientemente pequeno. Isto significa que os conjuntos $G_{o}=\{\lambda \in \mathbb{C}$ : $R(T+\lambda A) \in \operatorname{Cod}(Y)\}$ e $G_{1}=\{\lambda \in \mathbb{C}: R(T+\lambda A) \notin \operatorname{Cod}(Y)\}$ são abertos.

Proposição 163. Seja X um espaço de Banach. Então todo operador $S \in S S(X)$ é um operador de Riesz.

Prova. Seja $I$ o operador identidade sobre $X$. Segue da Proposição 161 que o operador $I-\lambda S$ é uma $\Phi$-injeção para todo número complexo $\lambda$. 
Dado $\lambda_{o} \in \mathbb{C}$ existe $M \in \operatorname{Cod}(X)$ tal que $\left(I-\lambda_{o} S\right) J_{M}^{X}$ é uma injeção. Seja $\lambda \in \mathbb{C}$ satisfazendo a relação

$$
\left|\lambda-\lambda_{o}\right||| S||<j\left(\left(I-\lambda_{o} S\right) J_{M}^{X}\right) .
$$

Segue, pelo Lema 162 , que $R\left(\left(I-\lambda_{o} S\right) J_{M}^{X}\right)$ e $R\left(\left(I-\lambda_{o} S\right) J_{M}^{X}-\lambda S+\lambda_{o} S\right)$ são subespaços de codimensão finita (infinita) em $X$ simultaneamente, ou seja, os subespaços $R((I-$ $\left.\left.\lambda_{o} S\right) J_{M}^{X}\right)$ e $R\left((I-\lambda S) J_{M}^{X}\right)$ são simultaneamente de codimensão finita (infinita) em $X$. Essa afirmação também é verdadeira para os subespaços $R\left(I-\lambda_{\circ} S\right)$ e $R(I-\lambda S)$.

Sejam $G_{o}=\left\{\lambda \in \mathbb{C}: R\left(I-\lambda S^{\prime}\right) \in \operatorname{Cod}(X)\right\}$ e $G_{1}=\{\lambda \in \mathbb{C}: R(I-\lambda S) \notin \operatorname{Cod}(X)\}$. Pelo que vimos na demonstração do Lema 162 os conjuntos $G_{0}$ e $G_{1}$ são abertos. Além disso, $G_{o} \cap G_{1}=\emptyset$ e $G_{o} \cup G_{1}=\mathbb{C}$. Como $\mathbb{C}$ é conexo então ou $G_{o}=\emptyset$ ou $G_{1}=\emptyset$. Como $0 \in G_{o}$ então segue que $G_{1}=\emptyset$. Portanto, $I-\lambda S \in \Phi(X), \forall \lambda \in \mathbb{C}$.

Observação 164. S'eja $X$ um espaço de Banach. Então todo operador inessencial de $X$ em $X$ é um operador de Riesz em $X$.

A proposição a seguir fornece uma proveitosa caracterização dos operadores inessenciais.

Proposição 165. Sejam $X$ e $Y$ espaços de Banach. Um operador $S \in L(X, Y)$ é inessencial se, e somente se, o operador $I_{X}-V S$ possui núcleo de dimensão finita para todo operador $V \in L(Y, X)$, onde $I_{X}$ denota o operador identidade sobre o espaço $X$.

Prova. Segue da definição de operadores inessenciais que se $S \in \operatorname{In}(X, Y)$ então $I_{X}-V S$ tem núcleo de dimensão finita para todo operador $V \in L(Y, X)$.

Reciprocamente, suponhamos que exista $V \in L(Y, X)$ tal que $R\left(I_{X}-V S\right)$ não é fechado. Então pela Proposição 49 para qualquer $\varepsilon>0$ existe um subespaço de dimensão infinita $M$ de $X$ tal que $\left(I_{X}-V S\right)_{\left.\right|_{M}}$ é aproximável (compacto) e $\left\|\left(I_{X}-V S\right)_{\left.\right|_{M}}\right\| \leq \varepsilon$. A prova desta proposição mostra ainda que o operador $\left(I_{X}-V S\right)_{\left.\right|_{M}}$ é nuclear. Para simplificar a notação denotaremos por $J$ a inclusão natural $J_{M}^{X}$ de $M$ em $X$. Coloquemos $P_{o}=\left(I_{X}-V S\right) J$. Então o operador $P_{o} \in L(M, X)$ é nuclear, isto é, existem funcionais lineares $x_{1}^{*}, x_{2}^{*}, x_{3}^{*}, \ldots \in M^{*}$ e $y_{1}, y_{2}, y_{3}, \ldots \in F$ tais que $P_{o}(x)=\sum_{i=1}^{\infty} x_{i}^{*}(x) y_{i}$ e $\sum_{i=1}^{\infty}\left\|x_{i}^{*}\right\|\left\|y_{i}\right\|<\infty$.

Pelo Teorema de Hahn-Banach cada funcional $x_{i}^{*}: M \rightarrow \mathbb{R}$ pode ser extendido a um funcional $f_{i}: X \rightarrow \mathbb{R}$, com $\left\|x_{i}^{*}\right\|=\left\|f_{i}\right\|, \forall i=1,2,3, \ldots$ Deste modo $P_{o}$ admite uma 
extensão nuclear $P \in L(X)$. Desde que todo operador nuclear é compacto (ver Observação 85) então $P \in K(X)$. Como $K(X) \subset K(X)^{\text {Rad }}$ ( veja. Proposição 92) então, em particular, existem operadores $U, H \in L(X)$ tais que $U\left(I_{X}-P\right)=I_{X}-H$, onde $H$ é um operador de posto finito. Como

$$
\left(I_{X}-H\right) J=U\left(I_{X}-P\right) J=U(J-P J)=U\left(J-P_{o}\right)=U V S J
$$

então $\left(I_{X}-U V S\right) J=H J$, implicando que $N(H J) \subset N\left(I_{X}-(U V) S\right)$ e então $\operatorname{dim}(N(H J))<$ $\infty$, visto que, por hipótese, $\operatorname{dim}\left(N\left(I_{X}-(U V) S\right)\right)<\infty$. Desde que $H J: M \rightarrow X$ tem posto finito e $\operatorname{dim}(M)=\infty$ então segue que $\operatorname{dim}(N(H J))=\infty$, que é uma contradição. Deste modo, $R\left(I_{X}-V S\right)$ é fechado, $\forall V \in L(Y, X)$. Portanto fica provado que qualquer operador $I_{X}-\lambda V S \operatorname{com} \lambda \in \mathbb{C}$ tem núcleo $N\left(I_{X}-\lambda V S\right)$ de dimensão finita e imagem $R\left(I_{X}-\lambda V S\right)$ fechada. Segue pelos mesmos argumentos da prova da Proposição 163 que os conjuntos $G_{o}=\left\{\lambda \in \mathbb{C}: \operatorname{codim}\left(R\left(I_{X}-\lambda V S\right)\right)<\infty\right\}$ e $G_{1}=\left\{\lambda \in \mathbb{C}: \operatorname{codim}\left(R\left(I_{X}-\lambda V S\right)\right)=\infty\right\}$ são abertos. Como $\mathbb{C}=G_{o} \cup G_{1}, G_{o} \cap G_{1}=\emptyset$ e $\mathbb{C}$ é conexo então ou $G_{o}=\emptyset$ ou $G_{1}=\emptyset$. Desde que $0 \in G_{o}$ então $G_{1}=\emptyset$. Em particular, $1 \in G_{o}$ e portanto $S \in \operatorname{In}(X, Y)$.

A classe In é simétrica no seguinte sentido:

Proposição 166. Sejam $X$ e $Y$ espaços de Banach. Então $L(X, Y)=\operatorname{In}(X, Y)$ se, $e$ somente se, $L(Y, X)=\operatorname{In}(Y, X)$.

Prova. Suponhamos que $T \in L(X, Y)-\operatorname{In}(X, Y)$. Pela Proposição 165 existe um operador $S \in L(Y, X)$ tal que $N\left(I_{X}-S T\right)$ é de dimensão infinita. Mostraremos que $S \in L(Y, X)-\operatorname{In}(Y, X)$. Se $x \in N\left(I_{X}-S T\right) \cap N(T)$ então $x=S T(x)$ e $T(x)=0$ implicando que $x=0$. Então

$$
N\left(I_{X}-S T\right) \cap N(T)=\{0\}
$$

Além disso se $x \in N\left(I_{X}-S T\right)$ então $0=T\left(I_{X}-S T\right)(x)=(T-T S T)(x)=$ $\left(I_{Y}-T S^{\prime}\right)(T(x))$, implicando que

$$
T(x) \in N\left(I_{Y}-T S\right)
$$

Como $\operatorname{dim}\left(N\left(I_{X}-S T\right)\right)=\infty$ então existe uma quantidade infinita de vetores $x_{1}, x_{2}, \ldots$ linearmente independentes em $N\left(I_{X}-S T\right)$ que geram este subespaço. Deste modo, se $\alpha_{1} x_{k 1}+\cdots+\alpha_{n} x_{k n}=0$ então $\alpha_{i}=0, \forall i=1, \ldots, n$, onde $k n \in \mathbb{N}$. Segue deste 
fato que os vetores $T\left(x_{1}\right), T\left(x_{2}\right), T\left(x_{3}\right), \ldots$ são linearmente independentes em $R(T)$. De fato, se $\alpha_{1} T\left(x_{k 1}\right)+\cdots+\alpha_{n} T\left(x_{k n}\right)=0$ então $T\left(\alpha_{1} x_{k 1}+\cdots+\alpha_{n} x_{k n}\right)=0$, implicando, por (5.4), que $\alpha_{1} x_{k 1}+\cdots+\alpha_{n} x_{k n} \in N\left(I_{X}-S T\right) \cap N(T)=\{0\}$. Logo

$$
\alpha_{1} x_{k 1}+\cdots+\alpha_{n} x_{k n}=0 \Rightarrow \alpha_{i}=0, \forall i=1, \ldots, n,
$$

onde $k n \in \mathbb{N}$. Portanto, $\operatorname{dim}\left(T\left(N\left(I_{X}-S T\right)\right)\right)=\infty$. Além disso, segue de (5.5) que $T\left(N\left(I_{X}-S T\right)\right) \subset N\left(I_{Y}-T S\right)$, implicando que $\operatorname{dim}\left(N\left(I_{Y}-T S\right)=\infty\right.$. Deste modo, segue da. Proposição 165 que $S \notin \operatorname{In}(Y, X)$.

Lema 167. Sejam $X$ e $Y$ espaços de Banach. Se $T \in L(X, Y)$ e $S \in L(Y, X)$ então

(i) $\alpha\left(I_{X}-S T\right)=\alpha\left(I_{Y}-T S\right)$;

(ii) $\beta\left(I_{X}-S T\right)=\beta\left(I_{Y}-T S\right)$;

(iii) $\bar{\beta}\left(I_{X}-S T\right)=\bar{\beta}\left(I_{Y}-T S\right)$.

Prova. (i) Se $x \in N\left(I_{X}-S T\right)$ então $x=S T(x)$, implicando que

$$
\left(I_{Y}-T S\right)(T(x))=T(x)-T S T(x)=T S T(x)-T S T(x)=0
$$

e portanto $T(x) \in N\left(I_{Y}-T S\right)$. Isto implica que $T\left(N\left(I_{X}-S T\right)\right) \subseteq N\left(I_{Y}-T S\right)$.

Por outro lado, seja $y \in N\left(I_{Y}-T S\right)$. Então $y=T S(y)$. Temos que

$$
\left(I_{X}-S T\right)(S(y))=S(y)-S T S(y)=S(y)-S(y)=0
$$

ou seja, $S(y) \in N\left(I_{X}-T S\right)$ e então $y \in T\left(N\left(I_{X}-S T\right)\right)$. Logo $S\left(N\left(I_{Y}-T S\right)\right) \subseteq$ $N\left(I_{X}-S T\right)$.

Consideremos o operador injetor $\widehat{T}: N\left(I_{X}-S T\right) \rightarrow N\left(I_{Y}-T S\right)$ induzido por $T$, definido por $\widehat{T}(x)=T(x), \forall x \in N\left(I_{X}-S T\right)$, e também o operador $\widehat{S}: N\left(I_{Y}-T S\right) \rightarrow$ $N\left(I_{X}-S T\right)$ induzido por $S$, definido por $\widehat{S}(y)=S(y), \forall y \in N\left(I_{Y}-T S\right)$. O operador $\widehat{T}$ é inversível com inversa $\widehat{S}$. De fato, seja $x \in N\left(I_{X}-S T\right)$, isto é, $x=S T(x)$. Temos que $\widehat{S} \widehat{T}(x)=\widehat{S}(T S T(x))=\operatorname{STSTST}(x)=x$, pois dado $y \in N\left(I_{Y}-T S\right)$ temos que $y=T S(y)$ e $\widehat{S}(y)=S T S(y)$. Por outro lado, dado $y \in N\left(I_{Y}-T S\right)$ temos que

$$
\widehat{T} \widehat{S}(y)=\widehat{T}(\operatorname{STS}(y))=\operatorname{TSTSTS}(y)=y .
$$

Isto prova (i).

Para provar (ii) notemos que se $x \in\left(I_{X}-S T\right)(X)$ então existe $e \in X$ tal que $x=$ $e-S T(e)$. Temos que $T(x)=T(e)-T S T(e)=\left(I_{Y}-T S\right)(T(e)) \in\left(I_{Y}-T S\right)(Y)$, 
implicando que

$$
T\left(\left(I_{X}-S T\right)(X)\right) \subseteq\left(I_{Y}-T S\right)(Y)
$$

e que o operador induzido $T^{\vee}: X /\left(I_{X}-S T\right)(X) \rightarrow Y /\left(I_{Y}-T S\right)(Y)$, definido por $T^{\vee}([x])=[T(x)], \forall[x] \in X /\left(I_{X}-S T\right)(X)$, é inversível, com inversa $S^{\vee}: Y /\left(I_{Y}-T S\right)(Y) \rightarrow$ $X /\left(I_{X}-S T\right)(X)$ induzida por $S$. Esse fato prova (ii). O mesmo argumento aplicado aos fechos das imagens provam (iii).

Corolário 168. Sejam $X$ e $Y$ espaços de Banach. Então $T \in \operatorname{In}(X, Y)$ se, e somente se, $I_{Y}-T S \in \Phi(Y), \forall S \in L(Y, X)$.

Prova. É uma conseqüência imediata da Proposição 166 e do Lema 167.

Lema 169. Sejam $X$ um espaço de Banach e $N$ um subespaço fechado de $X$. Sejam $T \in L(X)$, com imagem $R(T)$ fechada e $Q \in L(X, X / N)$, onde $Q$ denota a aplicação quociente canônica, definida por $Q(x)=[x], \forall x \in X$. Então

$$
\operatorname{codim}(R(T)) \geq \operatorname{codim}(Q(R(T))) \text {. }
$$

Prova. Se $\operatorname{codim}(R(T))<\infty$ então existe um subespaço $W$ de $X \operatorname{com} \operatorname{dim}(W)<\infty$ tal que

$$
X=T(X) \oplus W
$$

Digamos que $\operatorname{dim}(W)=n$. Aplicando $Q$ a ambos os membros de 5.6 obtemos

$$
X / N=Q(X)=Q(R(T))+Q(W) .
$$

A aplicação quociente canônica $Q$ é obviamente sobrejetora, implicando que

$$
\operatorname{codim}(Q(R(T))) \leq n=\operatorname{codim}(R(T)) .
$$

A próxima proposição dá uma caracterização dual da caracterização dos operadores inessenciais da Proposição 165.

Proposição 170. Sejam $X$ e $Y$ espaços de Banach. Então $T \in \operatorname{In}(X, Y)$ se, e somente se, $\bar{\beta}\left(I_{Y}-T S\right)<\infty, \forall S \in L(Y, X)$.

Prova. Obviamente $\bar{\beta}\left(I_{Y}-T S\right) \leq \beta\left(I_{Y}-T S\right)$ e deste modo, pelo o Corolário 168, se $T \in \operatorname{In}(X, Y)$ então $\bar{\beta}\left(I_{Y}-T S\right)<\infty$. 
Reciprocamente, suponhamos que $\bar{\beta}\left(I_{Y}-T S\right)<\infty, \forall S \in L(Y, X)$. Mostraremos que $I_{Y}-T S \in \Phi(Y)$ e daí concluiremos que $T \in \operatorname{In}(X, Y)$. Afirmamos que $\beta\left(I_{Y}-T S\right)<\infty$. De fato, se $\beta\left(I_{Y}-T S\right)=\infty$ então existe $S_{1} \in L(Y, X)$ tal que $\beta\left(I_{Y}-T S_{1}\right)=\infty$. Segue pela Proposição 63 que existe um subespaço fechado $N$ de codimensão infinita de $Y$ e um operador compacto $K: Y \rightarrow Y$ tais que

$$
\pi_{N} K=\pi_{N}\left(I_{F}-T S_{1}\right)
$$

onde $\pi_{N}$ denota a aplicação quociente canônica de $Y$ em $Y / N$ definida por $\pi_{N}(y)=$ $[y], \forall y \in Y$. Como $I_{Y} \in \Phi(Y)$ e $i\left(I_{Y}\right)=0$, onde $I_{Y}$ denota o operador identidade sobre $Y$, então pela Proposição 104 temos que $I_{Y}-K \in \Phi(Y)$. Segue da Proposição 120 que existem operadores $U \in L(Y)$ e $P \in \mathcal{F}(Y)$ tais que

$$
\left(I_{Y}-K\right) U=I_{Y}-P
$$

Compondo ambos os membros de (5.7) por $U$ obtemos

$$
\pi_{N} K U=\pi_{N}\left(I_{Y}-T S_{1}\right) U=\pi_{N} U-\pi_{N} T S_{1} U
$$

implicando que

$$
\pi_{N} T S_{1} U=\pi_{N} U-\pi_{N} K U=\pi_{N}\left(I_{Y}-K\right) U .
$$

Segue de (5.8) que $\pi_{N} T S_{1} U=\pi_{N}\left(I_{Y}-P\right)=\pi_{N}-\pi_{N} P$ implicando que $\pi_{N} P=$ $\pi_{N}\left(I_{Y}-T S_{1} U\right)$. Como $\pi_{N} P$ tem posto finito então sua imagem é fechada, implicando que $\pi_{N}\left(I_{Y}-T S_{1} U\right)(Y)=\overline{\pi_{N}\left(I_{Y}-T S_{1} U\right)(Y)}$.

Então pelos Lemas 165 e 169 segue que

$$
\begin{aligned}
\operatorname{codim}\left(\left(I_{Y}-T S_{1} U\right)(Y)\right) & \geq \operatorname{codim}\left[\pi_{N}\left(I_{Y}-T S_{1} U\right)\right](Y)= \\
& =\operatorname{codim} \overline{\left[\pi_{N}\left(I_{Y}-T S_{1} U\right)\right](Y)}= \\
& =\operatorname{codim}\left(\pi_{N} P(Y)\right)=\infty
\end{aligned}
$$

Portanto $\bar{\beta}\left(I_{Y}-T S_{1} U\right)=\infty$, que é uma contradição. Então $\beta\left(I_{Y}-T S\right)<\infty, \forall S \in$ $L(Y, X)$. Isto implica que dado $S \in L(Y, X)$ temos que $\beta\left(\lambda I_{F}-T S\right)<\infty, \forall \lambda \neq 0$ e deste modo $T S$ é um operador de Riesz sobre $Y$. Logo, $I_{Y}-T S \in \Phi(Y), \forall S \in L(Y, X)$. 


\subsection{Operadores improjetivos}

Nessa seção apresentaremos o conceito de operadores improjetivos, que foi introduzido por Tarafdar, E. em [29]. Apresentaremos os principais resultados acerca desse conceito. Dentre tais resultados destacamos uma importante caracterização geométrica dos espaços de Banach para os quais todo operador linear contínuo atuando entre estes espaços é improjetivo (veja 180).

Definição 171. Sejam $X$ e $Y$ espaços de Banach. Um operador linear contínuo $T: X \rightarrow$ $Y$ é dito improjetivo se não existe um subespaço de dimensão infinita $M \subset X$ tal que a restrição $T_{I_{M}}$ de $T$ a $M$ é um isomorfismo sobre a imagem e $T(M)$ é um subespaço complementado em $Y$.

Denotaremos por $\operatorname{Imp}(X, Y)$ a classe de todos os operadores improjetivos de $X$ em $Y$ e por I $m p$ a classe de todos os operadores improjetivos entre todos os espaços de Banach.

Observação 172. Obviamente todo operador estritamente singular é um operador improjetivo, isto é, $S S \subset \operatorname{Imp}$.

Observação 173. A inclusão SS $\subset$ Imp é estrita. De fato, consideremos uma isometria $T: l_{2}(\mathbb{N}) \rightarrow C[0,1]$ sobre a imagem. Temos que $T$ é improjetivo mas $T \notin$ $S S\left(l_{2}(\mathbb{N}), C[0,1]\right)$. Com efeito, suponhamos que $T$ não é improjetivo. Então existe um subespaço fechado de dimensão infinita $M \subset l_{2}(\mathbb{N})$ tal que a restrição $T_{\left.\right|_{M}}$ de $T$ a $M$ é um isomorfismo e $T(M)$ é um subespaço complementado em $C[0,1]$. Como $M$ é um subespaço fechado de $l_{2}(\mathbb{N})$ então $M$ é reflexivo. Daí $T(M)$ é reflexivo e então pela Proposição 20 $T(M)$ não pode ser complementado em $C[0,1]$. Uma contradição.

Definição 174. Um subconjunto $\mathcal{A} \subset L$ é um quase-ideal de operadores se:

(i) $\mathcal{F} \subset \mathcal{A}$, onde $\mathcal{F}$ é o conjunto de todos os operadores de posto finito;

(ii) Sejam $X, Y, Z$ e $W$ espaços de Banach. Se $A \in L(W, X), K \in \mathcal{A}(X, Y)$ e $B \in L(Y, Z)$ então $B K A \in \mathcal{A}(W, Z)$.

Um quase-ideal de operadores $\mathcal{A}$ é dito próprio se não existe um espaço de Banach de dimensão infinita $X$ tal que o operador identidade $I_{X} \in \mathcal{A}$. Um quase-ideal de operadores $\mathcal{A}$ é um ideal de operadores em $L$ se $\mathcal{A}(X, Y)$ é um subespaço de $L(X, Y)$ para quaisquer espaços de Banach $X$ e $Y$. 
Lema 175. Sejam $X$ e $Y$ espaços de Banach. Se $T: X \rightarrow Y$ é um operador linear contínuo tal que a restrição $T_{\left.\right|_{M}}$ de $T$ a um subespaço fechado $M$ de $X$ é um isomorfismo e $T(M)$ é complementado em $Y$ então $M$ é complementado em $X$.

Prova. Seja $P$ uma projeção contínua de $Y$ sobre $T(M)$ e seja $\varphi: T(M) \rightarrow M$ a inversa da restrição $T_{\left.\right|_{M}}$.

$$
X \stackrel{T}{\rightarrow} Y \stackrel{P}{\rightarrow} T M \stackrel{\varphi}{\rightarrow} M
$$

Temos que $(\varphi P T)(\varphi P T)=\varphi P T$ implicando que $\varphi P T$ é uma projeção de $X$ em $M$. Portanto $M$ é complementado em $X$.

Proposição 176. Sejam $X$ e $Y$ espaços de Banach. Se $T: X \rightarrow Y$ é um operador improjetivo então T B e CT também são operadores improjetivos, para quaisquer operadores $B \in L(Z, X) \in C \in L(Y, W)$.

Prova. Suponhamos que $T B \notin \operatorname{Imp}(Z, Y)$. Então existe um subespaço fechado de dimensão infinita $M$ de $Z$ tal que a restrição $(T B)_{\left.\right|_{M}}: M \rightarrow T B(M)$, de $T B$ a $M$, é um isomorfismo e $T B(M)$ é complementado em $Y$. Então existem números reais $N>0$ e $K>0$ tais que

$$
N\|m\| \leq\|T B(m)\| \leq K\|m\|, \forall m \in M,
$$

implicando que $N\|m\| \leq\|T B(m)\| \leq\|T\|\|B(m)\|$ e isso implica que

$$
\frac{N}{\|T\|}\|m\| \leq\|B(m)\|, \forall m \in M .
$$

Portanto, a restrição $B_{\left.\right|_{M}}$, de $B$ a $M$, é um isomorfismo. $\operatorname{Logo}, \operatorname{dim}(B(M))=\infty$ e $B(M)$ é um subespaço fechado de $X$. Temos que para qualquer $y \in B(M)$ existe um $x \in M$ tal que $B(x)=y$. Então

$$
\|T(y)\|=\|T B(x)\| \geq N\|x\| \geq N\|B\|^{-1}\|y\| .
$$

Assim, a restrição $T_{\left.\right|_{B(M)}}$ de $T$ a $B(M)$ é um isomorfismo sobre a imagem e $T B(M)$ é complementado em $Y$, implicando que $T$ não é improjetivo, o que contradiz a hipótese. De modo análogo verificamos que $C T$ é improjetivo.

Uma conseqüencia imediata da proposição anterior é que a classe Imp de todos os operadores improjetivos é um quase-ideal de operadores. A seguir exibiremos uma proveitosa caracterização dos operadores improjetivos. Para tal utilizaremos o seguinte lema. 
Lema 177. Sejam $X$ e $Y$ espaços de Banach $\in T \in L(X, Y)$.

(i) Se $M$ é um subespaço fechado de $X$ tal que $T J_{M}^{X}$ é um isomorfismo, $T(M)$ é complementado em $Y$ e $N$ é o complemento fechado de $T(M)$ então $M$ é complementado em $X$ e $T^{-1}(N)$ é o complemento fechado de $M$;

(ii) Se $N$ é um subespaço fechado de $Y$ tal que $Q_{N} T$ é sobrejetivo, $T^{-1}(N)$ é complementado em $X$ e $M$ é o complemento fechado de $T^{-1}(N)$ então $N$ é complementado em $Y$ e $T(M)$ é o complemento fechado de $N$, onde $Q_{N}: Y \rightarrow Y / N$ é a aplicação quociente canônica definida por $Q_{N}(y)=[y], \forall y \in Y$.

Prova. Temos que $T^{-1}(N) \cap M=\{0\}$ pois se $x \in T^{-1}(N) \cap M=\{0\}$ então $T(x) \in$ $T(M) \cap N=\{0\}$, implicando que $T(x)=0$. Como $T J_{M}^{X}$ é um isomorfismo então $x=0$. Além disso, como $T \in L(X, Y)$ e $N$ é um subespaço fechado então $T^{-1}(N)$ é um subespaço fechado. Portanto, $X=T^{-1}(N) \oplus M$ e isto prova (i).

Para provar (ii) observemos inicialmente que $T(M) \cap N=\{0\}$. De fato, se $y \in T(M) \cap$ $N$ então existe $m \in M$ tal que $y=T(m) \in N$. Isto implica que $m \in T^{-1}(N) \cap M$ e portanto $m=0$. Assim, $y=T(m)=0$. Portanto temos que $T(M) \cap N=\{0\}$. Observemos ainda que $T(M) \oplus N=T(X)+N=Y$. Com efeito, temos que

$$
X \stackrel{T}{\rightarrow} T(X) \subset Y \stackrel{Q_{N}}{\rightarrow} Y / N .
$$

Dado $[y] \in Y / N$, como $Q_{N} T$ é sobrejetor então existe $x \in X$ tal que $[y]=Q_{N} T(x)$. Em particular, $y=T(x)+n$, onde $n \in N$. Como $N$ é fechado então $T(M)$ também é um subespaço fechado e isto prova (ii).

Proposição 178. Sejam $X$ e $Y$ espaços de Banach. Um operador $T \in \operatorname{Imp}(X, Y)$ se, e somente se, não existe um subespaço fechado de codimensão infinita $N$ de $Y$ tal que $Q_{N} T$ é sobrejetor e $T^{-1}(N)$ é um subespaço complementado de $X$.

Prova. Suponhamos que $T \in \operatorname{Imp}(X, Y)$ e que $N$ seja um subespaço fechado de $Y$ tal que $Q_{N} T$ é sobrejetor e $T^{-1}(N)$ é complementado em $X$. Mostraremos que neste caso $N$ é de codimensão finita. Segue do Lema 177 que $N$ é complementado e $T(M)$ é o complemento fechado de $N$, isto é, $Y=T(M) \oplus N$. Seja $x \in M$ tal que $T(x)=0$. Como $T(x)=0 \in N$ então temos que $x \in T^{-1}(N)$. Então $x \in M$ então $x \in M \cap T^{-1}(N)=\{0\}$, ou seja, $x=0$. Portanto, a restrição $T_{\left.\right|_{M}}$ de $T$ a $M$ é injetora. Segue do Teorema da aplicação aberta que a restrição $T_{\left.\right|_{M}}$ é um isomorfismo. Como $T$ é improjetivo segue que $T(M)$ tem dimensão finita. Deste modo, temos que $\operatorname{codim}(N)<\infty$ em $Y$. 
Reciprocamente, suponhamos que $T \notin \operatorname{Imp}(X, Y)$. Então existe um subespaço fechado de dimensão infinita $M$ de $X$ tal que o operador $T J_{M}^{X}$ é um isomorfismo e $T(M)$ é complementado em $Y$. Seja $N$ o complemento fechado de $T(M)$. Então $Y=T(M) \oplus N$. Como $\operatorname{dim}(M)=\infty$ e $T J_{M}^{X}$ é um isomorfismo então $\operatorname{dim}(T(M))=\infty$, implicando que $\operatorname{codim}(N)=\infty \mathrm{em} Y$. Além disso, temos que $Q_{N} T$ é sobrejetor. Com efeito, se $[y] \in Y / N$ então existe $m \in M$ e $N_{o} \in N$ tais que $y=T(m)+n_{o}$. Aplicando $Q_{N}$ em ambos os membros dessa igualdade obtemos

$$
Q_{N}(y)=Q_{N} T(m)+Q_{N}\left(n_{o}\right)=Q_{N} T(m) \Rightarrow[y]=Q_{N}(y)=Q_{N} T(m) .
$$

Então segue do Lema 177 que $M$ é um complemento fechado de $T^{-1}(N)$, que é uma contradição. Logo, o operador $T$ é improjetivo.

Definição 179. Dois espaços de Banach $X$ e $Y$ são ditos projeção totalmente incomparáveis se não existe em $X$ um subespaço complementado de dimensão infinita que seja isomorfo a um subespaço complementado de $Y$.

Proposição 180. Sejam $X$ e $Y$ dois espaços de Banach. Então $L(X, Y)=\operatorname{Imp}(X, Y)$ se, e somente se, $X$ e $Y$ são projeção totalmente incomparáveis.

Prova. Suponhamos que $L(X, Y)=\operatorname{Imp}(X, Y)$. Suponhamos também que $X$ e $Y$ não são projeção totalmente incomparáveis. Então existem subespaços de dimensão infinita $Z$ e $U$ em $X$ e $Y$, respectivamente, com $Z$ complementado em $X$ e $U$ complementado em $Y$ tais que $Z \sim U$. Digamos que $j: Z \rightarrow U$ é um isomorfismo entre tais subespaços. Seja $P: X \rightarrow Z$ uma projeção sobre $Z$. Definimos o operador $\widetilde{T}: X \rightarrow Y$ por $\widetilde{T}(x)=$ ijP $P(x), \forall x \in X$, onde $i: U \rightarrow Y$ é a inclusão canônica de $U$ em $Y$ definida por $i(y)=$ $y, \forall y \in U$.Cono a composição de operadores contínuos é também um operador contínuo, então $\widetilde{T} \in L(X, Y)$. Além disso, a restrição $\widetilde{T}_{\mid z}$, de $\widetilde{T}$ a $Z$, é um isomorfismo. De fato, já observamos que $\widetilde{T}$ é contínuo. Além disso, $\widetilde{T}$ é injetor, pois se $z \in Z$ e $\widetilde{T}(z)=0$ então $0=\widetilde{T}(z)=i j P(z)=i j(z)$, implicando que $j(z)=0$. Deste modo, $z=0$, pois $j$ é um isomorfismo. Então pelo Teorema da aplicação aberta, a inversa de $\widetilde{T}$ é contínua. Logo, $\widetilde{T}$ é um isomorfismo.

Temos ainda que $\tilde{T}(Z)=i j P(Z)=i j(Z)=i(U)=U$. Logo, $\widetilde{T}(Z)$ é complementado em $Y$, implicando que $\widetilde{T} \in L(X, Y)-\operatorname{Imp}(X, Y)$, que é uma contradição. Logo, $X$ e $Y$ são projeção totalmente incomparáveis.

Reciprocamente, suponhamos que $X$ e $Y$ sejam projeção totalmente incomparáveis. 
Seja $T \in L(X, Y)-\operatorname{Imp}(X, Y)$. Então existe um subespaço fechado de dimensão infinita $M$ de $X$ tal que a restrição $T_{\left.\right|_{M}}$, de $T$ a $M$, é um isomorfismo e o subespaço $T(M)$ é complementado em $Y$. Segue do Lema 175 que $M$ é um subespaço complementado em $X$, o que contradiz a hipótese. $\operatorname{Logo}, T \in \operatorname{Imp}(X, Y)$, implicando que $L(X, Y)=\operatorname{Imp}(X, Y)$.

\subsection{Operadores inessenciais e improjetivos}

Veremos nesta seção que a classe de todos os operadores inessenciais está contida na classe de todos os operadores improjetivos. Apresentaremos também alguns resultados de [3] que mostram alguns casos em que estas classes de operadores coincidem. Contudo, como veremos na seção seguinte, existem espaços de Banach $X$ e $Y$ e existe um operador linear contínuo de $X$ em $Y$ que é improjetivo mas não é inessencial.

Proposição 181. Sejam $X$ e $Y$ espaços de Banach. Então $\operatorname{In}(X, Y) \subset \operatorname{Imp}(X, Y)$.

Prova. Sejam $X$ e $Y$ espaços de Banach e $T \in L(X, Y)-\operatorname{Imp}(X, Y)$. Então existe um subespaço fechado $M$ de $X$ de dimensão infinita tal que $T J_{M}^{X}$ é um isomorfismo e $T(M)$ é um subespaço complementado em $Y$. Segue do Lema 177 que $M$ é complementado em $X$. Se $N$ é o complemento fechado de $T(M)$ em $Y$, isto é, se $Y=T(M) \oplus N$ então $X=M \oplus$ $T^{-1}(N)$. Temos que os subespaços $N$ e $T^{-1}(N)$ são fechados em $Y$ e $X$, respectivamente. Definimos $S: Y=T(M) \oplus N \rightarrow X$ por $S(T(m)+n)=\left(T_{\left.\right|_{M}}\right)^{-1}(T(m))=m$. Temos que se $y \in T(M)$ então $S(y)=\left(T_{\left.\right|_{M}}^{-1}\right)^{-1}(y)$ e se $y \in N$ então $S(y)=S(0+y)=S(0)=0$. Além disso,

$$
N\left(I_{X}-S T\right)=M
$$

De fato, se $x \in M$ então $\left(I_{X}-S T\right)(x)=x-S T(x)=x-\left(T_{\left.\right|_{M}}\right)^{-1}(T(x))=x-x=0$. Portanto, $M \subset N\left(I_{X}-S T\right)$. Por outro lado, se $x \in N\left(I_{X}-S T\right)$ então $\left(I_{X}-S T\right)(x)=0$ implicando que $x-S T(x)=0$. Daí segue que $x=S T(x)$. Digamos que $T(x) \notin T(M)$. Então $T x \in N$ e, portanto, $S(T(x))=0 \in M$ implicando que $x=S(T(x)) \in M$. Se $T(x) \in T(M)$ então, obviamente, $x \in M$.

Segue de (5.9) que $\operatorname{dim}\left(N\left(I_{X}-S T\right)\right)=\operatorname{dim}(M)=\infty$, implicando, pela Proposição 165 , que $T \notin \operatorname{In}(X, Y)$. Deste modo, $\operatorname{In}(X, Y) \subset \operatorname{Im} p(X, Y)$. 
Veremos na próxima seção que tal inclusão é estrita, ou seja., existem espaços de Banach $X$ e $Y$ e um operador $T \in L(X, Y)$ tais que $T$ é improjetivo mas não é inessencial.

A seguir apresentaremos alguns resultados que mostram que sob algumas condições ocorre a igualdade $I n=I m p$.

Lema 182. Sejam $X$ e $Y$ espaços de Banach, $T \in L(X, Y)$ e $S \in L(Y, X)$.

(i) Se o subespaço $M=N\left(I_{X}-S T\right)$ é complementado em $X$ com complemento fechado $U$ então ambos os subespaços $T(M)$ e $S^{-1}(U)$ são complementados em $Y$, isto é, $Y=$ $T(M) \oplus S^{-1}(U)$;

(ii) Se o subespaço $N=\overline{R\left(I_{Y}-T S\right)}$ é complementado em $Y$ com complemento fechado $V$ então ambos os subespaços $T^{-1}(N)$ e $S(V)$ são complementados em $X$, isto é, $X=$ $T^{-1}(N) \oplus S(V)$.

Prova. Em (i) o objetivo é definir uma projeção $Q$ em $Y$ e mostrar que

$$
N(Q)=S^{-1}(U) \text { e } R(Q)=T(M) .
$$

Consideremos uma projeção $P X \rightarrow M$. Então $\left(I_{X}-S T\right) P=0$, implicando que $P=S T P$. Definimos $Q=T P S: Y \rightarrow T(M) \subset Y$. Temos que

$$
Q^{2}=T P(S T P) S=T P^{2} S=T P S=Q
$$

e, portanto, $Q$ é uma projeção. Além disso,

$$
N(Q)=S^{-1}(U)
$$

Com efeito, se $x \in S^{-1}(U)$ então $S(x) \in U$, implicando que $P S(x)=0$ e deste modo, $x \in N(P S)$. Então $P S(x)=0$, implicando que $Q(x)=T P S(x)=T(0)=0$. Logo, $x \in$ $N(Q)$. Por outro lado, se $x \in N(Q)$ segue que $Q(x)=0$. Logo, $T P S(x)=0$, implicando que $P S(x)=S T P S(x)=S(0)=0$. Desse modo, $x \in N(P S)$. Então $P S(x)=0$ implica que $S(x) \in U$, que por sua vez implica que $x \in S^{-1}(U)$.

Temos também que

$$
R(Q)=T(M)
$$

De fato, temos que $Q(Y)=T P S(Y) \subseteq T(M)$, implicando que $R(Q) \subseteq T(M)$. Por outro lado, como $P=S T P$ então

$$
S T(M)=M .
$$


Portanto, se $x \in M$ então temos que existe $m \in M$ tal que $x=P(m)$, implicando por (5.12) a existência de um $m^{\prime} \in M$ tal que $m=S T\left(m^{\prime}\right)$ e $T(x)=(T P S) T\left(m^{\prime}\right) \in Q(Y)$. Assim, temos que $T(M) \subseteq R(Q)$. As igualdades (5.10) e (5.11) provam (i).

Verifiquemos (ii). Seja $Q: Y \rightarrow V$ uma projeção sobre $V$. Então $N(Q)=\overline{R\left(I_{Y}-T S\right)}$ implicando que $Q\left(I_{Y}-T S\right)=0$. Deste modo, $Q=Q T S$. Definindo $P=S Q T$ temos que $P^{2}=S(Q T S) Q T=S Q^{2} T=S Q T=P$ e, portanto, $P$ é uma projeção. Mostraremos que $T^{-1}(N)=N(P)$ e $S(V)=R(P)$. Temos que $N(S) \cap R(Q)=\{0\}$. De fato, seja $y \in N(S) \cap R(Q)$. Então $y \in N(S)$ implica que $S(y)=0$. Como $y \in R(Q)$ então $y=Q(y)=Q T S(y)=Q T(0)=0$. Mostremos que $N(P)=T^{-1}(N)$. Por um lado temos que se $x \in N(P)$ então $P(x)=0$, implicando que $S Q T(x)=0$. Então segue que $Q T(x) \in N(S) \in R(Q)=\{0\}$, implicando que $Q T(x)=0$. Isto implica que $x \in N(Q T)$ e portanto $N(P) \subset N(Q T)$. Por outro lado, se $x \in N(Q T)$ então $Q T(x)=0$, implicando que $P(x)=S Q T(x)=S(0)=0$. Então $x \in N(P)$ e portanto $N(Q T) \subset N(P)$.

Mostremos que $R(P)=S(V)$. Por um lado temos que se $y \in V$ então $y=Q(y)$, implicando que $S(y)=S Q(y)=(S Q T) S(y)=P(S(y)) \in R(P)$ e portanto $S(V) \subset R(P)$. Por outro lado, como $P=S Q T$ então $R(P) \subset S(V)$. Portanto, $X=N(P) \oplus R(P)=$ $S(V) \oplus T^{-1}(N)$.

Proposição 183. Sejam $X$ e $Y$ espaços de Banach e $T \in L(X, Y)$. São equivalentes as seguintes afirmações:

(i) $T \in \operatorname{In}(X, Y)$;

(ii) $T \in \operatorname{Imp}(X, Y)$ e $N\left(I_{X}-S T\right)$ é complementado em $X, \forall S \in L(Y, X)$;

(iii) $T \in \operatorname{Imp}(X, Y)$ e $N\left(I_{Y}-T S\right)$ é complementado em $Y, \forall S \in L(Y, X)$;

(iv) $T \in \operatorname{Imp}(X, Y)$ e $\overline{R\left(I_{X}-S T\right)}$ é complementado em $X, \forall S \in L(Y, X)$;

(v) $T \in \operatorname{Imp}(X, Y)$ e $\overline{R\left(I_{Y}-T S\right)}$ é complementado em $Y, \forall S \in L(Y, X)$.

Prova. $(i) \Rightarrow(i i),(i i i),(i v),(v)$.

Como $T \in \operatorname{In}(X, Y)$ então segue da Proposição 181 que $T \in \operatorname{Imp}(X, Y)$. Pelo Lema 167, pela Proposição 165 e pela Proposição 170 temos que $\operatorname{dim}\left(N\left(I_{X}-S T\right)\right)<$ $\infty, \operatorname{dim}\left(N\left(I_{Y}-T S\right)\right)<\infty, \operatorname{codim}\left(\overline{R\left(I_{X}-S T\right)}\right)<\infty$ e $\operatorname{codim}\left(\overline{R\left(I_{Y}-T S\right)}\right)<\infty$ e, além disso, tais subespaços são complementados.

(ii) $\Rightarrow($ i) .

Sejam $T \in \operatorname{Imp}(X, Y)$ e $M=N\left(I_{X}-S T\right)$ complementado em $X$, onde $S \in L(Y, X)$. 
Seja $x \in M$. Então $x=S T(x)$. Isto implica que se $T(x)=0$ então $x=S T(x)=S(0)=0$ implicando que $N\left(T_{\left.\right|_{M}}\right)=\{0\}$. Segue do Teorema da aplicação aberta que a restrição $T_{\left.\right|_{M}}$, de $T$ a $M$, é um isomorfismo. Pelo Lema 182 temos que $T(M)$ é um subespaço complementado em $Y$. Como $T \in \operatorname{Imp}(X, Y)$ então $\operatorname{dim}(M)<\infty$, implicando pelo Lema 167 e pela Proposição 165 que $T \in \operatorname{In}(X, Y)$.

(iii) $\Rightarrow($ i)

Sejam $T \in \operatorname{Imp}(X, Y)$ e $N=N\left(I_{Y}-T S\right)$ complementado em $Y$, onde $S \in L(Y, X)$. Se $y \in N$ então $y=T S(y)$. Se $y \in N$ e $S(y)=0$ então temos que $y=T S(y)=T(0)=0$ e isto implica que $N\left(S_{\left.\right|_{N}}\right)=\{0\}$. Pelo Teorema da aplicação aberta segue que $S$ é um isomorfismo sobre $N$ e portanto $S(N)$ é um subespaço fechado, pois $N$ é fechado.

Seja $x \in S(N)$. Então existe $n \in N\left(I_{Y}-T S\right)=N$ tal que $x=S(n)$. Se $T(x)=0$ e $x=S(n)$ então $x=S(T S(n))=S(T(x))=S(0)=0$ implicando que $N\left(T_{\left.\right|_{S(N)}}\right)=\{0\}$. Portanto $T$ é um isomorfismo sobre $S(N)$. Além disso, $T(S(N))=N$. De fato, se $y \in$ $T S(N)$ então existe $n \in N$ (e portanto $n=T S(n))$ tal que $y=T S(n)$. Daí, $\left(I_{Y}-T S\right)(y)=$ $y-T S(y)=T S(n)-T S(T S(n))=T S(T S(n))-T S(T S(n))=0$, implicando que $y \in N$ e portanto $T(S(N)) \subset N$. Por outro lado, $N \subset T S(N)$, implicando que $N=T(S(N))$ e $N$ é complementado em $Y$.. Como $T \in \operatorname{Im} p(X, Y)$ então $\operatorname{dim}(N)<\infty$, implicando, pelo Lema 167 e pela Proposição 170 , que $T \in \operatorname{In}(X, Y)$.

(iv) $\Rightarrow(i)$.

Sejam $T \in \operatorname{Imp}(X, Y)$ e $M=\overline{R\left(I_{X}-S T\right)}$ complementado em $X$, onde $S \in L(Y, X)$. Como $M \subset N\left(Q_{M}\right)$, onde $Q_{M}: X \rightarrow X / M$ é a aplicação quociente canônica definida por $Q_{M}(x)=[x]$, então $Q_{M}\left(I_{X}-S T\right)=0 \cdot$ implicando que $Q_{M}(S T)=Q_{M}$. Se $x \in X$ então temos que $[x]=Q_{M}(x)=Q_{M}(S T(x))=[S T(x)]$. Em particular, $S T(x)-x \in M$. Portanto, existe $m \in M$ tal que $S T(x)-x=m$, implicando que $x=S T(x)-m$. Assim,

$$
X=R(S T)+M
$$

Deste modo, $R(T)+S^{-1}(M)=Y$, implicando que $Q_{S^{-1}(M)} T$ é sobrejetor, pois dado $[y] \in Y / S^{-1}(M)$ temos que $[y]=Q_{S^{-1}}(y), y \in Y$. Como $Y=R(T)+S^{-1}(M)$, segue que existem $x \in X$ e $m^{\prime} \in S^{-1}(M)$ tais que $y=T(x)+m^{\prime}$. Pela linearidade de $Q_{S^{-1}(M)}$ segue que

$[y]=Q_{S^{-1}(M)}(y)=Q_{S^{-1}(M)}\left(T(x)+m^{\prime}\right)=Q_{S^{-1}(M)} T(x)+Q_{S^{-1}(M)}\left(m^{\prime}\right)=Q_{S^{-1}(M)} T(x)$.

Logo, $Q_{S^{-1}(M)} T$ é sobrejetor. Além disso, 


$$
\begin{gathered}
T^{-1} S^{-1}(M)=(S T)^{-1}(M)={ }^{\perp}\left((S T)^{*}\left(M^{\perp}\right)\right)= \\
={ }^{\perp}\left(T^{*} S^{*}\left(N\left(I_{X^{*}}-T^{*} S^{*}\right)\right)\right)={ }^{\perp}\left(N\left(I_{X^{*}}-T^{*} S^{*}\right)\right)=M .
\end{gathered}
$$

Como $M$ é complementado em $X$ então segue que $T^{-1}\left(S^{-1}(M)\right)$ é complementado em $X$. Segue da Proposição 178 que $S^{-1}(M)$ é de codimensão finita e portanto $T^{-1} S^{-1}(M)=$ $M$ é de codimensão finita. Logo, $\overline{R\left(I_{X}-S T\right)}$ é de codimensão finita, $\forall S \in L(Y, X)$. Então segue pelo Lema 167 que $T \in \operatorname{In}(X, Y)$.

$(v) \Rightarrow(i)$.

Sejam $T \in \operatorname{Imp}(X, Y)$ e $N=\overline{R\left(I_{Y}-T S\right)}$ complementado em $Y$, onde $S \in L(Y, X)$. Como $Q_{N}\left(I_{Y}-T S\right)=0$ segue que $Q_{N} T S=Q_{N}$, onde $Q_{N}$ é a aplicação quociente canônica de $Y$ em $Y / N$. Temos que se $y \in Y$ então $[y]=Q_{N}(y)=Q_{N}(T S(y))=[\operatorname{TS}(y)]$. Em particular, $T S(y)-y \in N$, implicando que existe $n \in N$ tal que $T S(y)-y=n$ e isto implica que $y=T S(y)-n$. Portanto, $Y=R(T S)+N$. Deste modo, $Q_{N} T$ é sobrejetor, pois dado $[y] \in Y / N$ temos que $[y]=Q_{N}(y)$ e como $Y=R(T S)+N$ então segue que existem $y^{\prime} \in Y$ e $n \in N$ tais que $y=T S\left(y^{\prime}\right)+n$. Pela linearidade de $Q_{N}$ segue que

$$
[y]=Q_{N}(y)=Q_{N}\left(T S\left(y^{\prime}\right)+n\right)=Q_{N}\left(T S\left(y^{\prime}\right)\right)+Q_{N}(n)=Q_{N}\left(T S\left(y^{\prime}\right)\right) .
$$

Além disso, segue do Lema 182 que $T^{-1}(N)$ é complementado em $X$. Como $T \in$ $\operatorname{Imp}(X, Y)$ então segue da Proposição 178 que $N$ é de codimensão finita em $Y$. Assim, pelo Lema 167 , temos que $T \in \operatorname{In}(X, Y)$.

Nesse estudo das relações entre as classes dos operadores inessenciais e improjetivos os autores González, M. e Aiena, P. [3] apresentaram as seguintes questões:

Questão 1. In $(X, Y)=\operatorname{Imp}(X, Y)$, para quaisquer espaços de Banach $X$ e $Y$ ?

Questão 2. $\operatorname{Imp}(X, Y)$ é um subespaço de $L(X, Y)$, para quaisquer espaços de Banach $X$ e $Y$ ?

A partir daí os autores apresentaram outra questão:

Questão 3. Fixado um par de espaços de Banach $X, Y$ é verdade que $\operatorname{In}(X, Y)=$ $\operatorname{Imp}(X, Y)$ sempre que $\operatorname{Imp}(X, Y)$ é um subespaço de $L(X, Y)$ ?

Os resultados a seguir simplificam a Questão 1, que trata de espaços de Banach distintos, para o caso de operadores atuando num único espaço de Banach.

Proposição 184. Sejam $X$ e $Y$ espaços de Banach. Então $\operatorname{In}(X, Y)=\operatorname{Imp}(X, Y)$ se, $e$ somente se, $\operatorname{In}(Y, X)=\operatorname{Imp}(Y, X)$. 
Prova. Suponhamos que $\operatorname{In}(X, Y)=\operatorname{Imp}(X, Y)$. Como $\operatorname{In}(Y, X) \subset \operatorname{Imp}(Y, X)$ então basta mostrarmos que $\operatorname{Imp}(Y, X) \subset \operatorname{In}(Y, X)$. Sejam $T \in \operatorname{Imp}(Y, X)$ e $S \in L(X, Y)$. Segue da Proposição 176 que $\operatorname{STS} \in \operatorname{Im} p(X, Y)=\operatorname{In}(X, Y)$. Como $\operatorname{In}(X, Y)$ é um ideal de operadores então

$$
\left(T S^{\prime}\right)^{2}=T(S T S) \in \operatorname{In}(X)
$$

e portanto,

$$
I_{X}-(T S)^{2}=\left(I_{X}+T S\right)\left(I_{X}-T S\right) \in \Phi(X),
$$

implicando que $\operatorname{dim}\left(N\left(I_{X}-(T S)^{2}\right)\right)<\infty$.

Se $x \in N\left(I_{X}-T S\right)$ então $x=T S(x)$, implicando que $\left(I_{X}-(T S)^{2}\right)(x)=x-$ $T S(T S(x))=x-T S(x)=x-x=0$. Isto implica que $x \in N\left(I_{X}-(T S)^{2}\right)$ e portanto $N\left(I_{X}-T S\right) \subset N\left(I_{X}-(T S)^{2}\right)$. Logo, o subespaço $N\left(I_{X}-T S\right)$ tem dimensão finita. Então segue pelo Lema 167 que $T \in \operatorname{In}(Y, X)$.

Lembremos que dado um subespaço fechado $M$ de um espaço de Banach $X$ e dado um operador $T: X \rightarrow X$, dizemos que $M$ é invariante por $T$ se $T(M) \subset M$. Consideremos os operadores lineares $T_{M}: M \rightarrow M$, definido por $T_{M}(m)=T(m)$ e $T^{M}: X / M \rightarrow X / M$ definido por $T^{M}([x])=[T(x)]$. Definimos

$\Omega_{+}(X)=\left\{T \in L(X): \nexists M\right.$ invariante, $\operatorname{dim}(M)=\infty$ tal que $T_{M}$ é um isomorfismo. $\}$

$\Omega_{-}(X)=\left\{T \in L(X): \nexists M\right.$ invariante, $\operatorname{codim}(\mathrm{M})=\infty$ tal que $T^{M}$ é sobrejetor. $\}$

Proposição 185. Seja $\mathcal{A}$ um quase-ideal de operadores. Então para qualquer espaço de Banach $Y$ temos que

(i) $\mathcal{A}(Y) \subset \Omega_{+}(Y)$ implica que $\mathcal{A}(Y, Z) \subset \operatorname{In}(Y, Z)$, para qualquer espaço de Banach $Z$;

(ii) $\mathcal{A}(Y) \subset \Omega_{-}(Y)$ implica que $\mathcal{A}(X, Y) \subset \operatorname{In}(X, Y)$, para qualquer espaço de Banach $X$.

Prova. Suponhamos que exista um espaço de Banach $Z$ tal que $\mathcal{A}(Y, Z) \not \subset \operatorname{In}(Y, Z)$. Então existe um operador $T \in \mathcal{A}(Y, Z)-\operatorname{In}(Y, Z)$. Segue do Lema 167 que existe um operador $S \in L(Z, Y)$ tal que $M=N\left(I_{Y}-S T\right)$ é de dimensão infinita. Como $S T \in$ $\mathcal{A}(Y) \subset \Omega_{+}(Y)$ e $(S T)_{\left.\right|_{M}}=I_{\left.\right|_{M}}$ então $M$ é de dimensão finita, que é uma contradição. Logo, $\mathcal{A}(Y, Z) \subset \operatorname{In}(Y, Z)$.

Provemos (ii). Suponhamos que exista um espaço de Banach $X$ tal que $\mathcal{A}(X, Y) \not \subset$ $\operatorname{In}(X, Y)$. Então existe um operador $T \in \mathcal{A}(X, Y)-\operatorname{In}(X, Y)$. Segue do Lema 167 que existe um operador $S \in L(Y, X)$ tal que $N=\overline{R\left(I_{Y}-T S\right)}$ é de codimensão infinita. Como 
$T S \in \mathcal{A}(Y) \subset \Omega_{-}(Y), N$ é invariante por $T S$ e $(T S)^{N}$ é sobrejetor então $\operatorname{codim}(N)<\infty$ em $Y$.

Corolário 186. Seja $X$ um espaço de Banach. São equivalentes as seguintes afirmações:

(i) $\operatorname{In}(X)=\operatorname{Imp}(X)$;

(ii) $\operatorname{Imp}(X) \subset \Omega_{+}(X)$;

(iii) $\operatorname{Imp}(X) \subset \Omega_{-}(X)$;

(iv) In $(X, Y)=\operatorname{Imp}(X, Y)$, para qualquer espaço de Banach $Y$.

Prova. (i) $\Rightarrow$ (ii) e $(i) \Rightarrow($ iii $)$.

Seja $T \in L(X) \backslash\left(\Omega_{+}(X) \cap \Omega_{-}(X)\right)$. Então ou $T \in L(X)-\Omega_{+}(X)$ ou $T \in L(X)-\Omega_{-}(X)$.

Suponhamos que $T \in L(X)-\Omega_{+}(X)$. Então existe um subespaço de dimensão infinita $M$ de $X$, com $T(M) \subset M$, tal que o operador $T_{M}: M \rightarrow M$ definido por $T_{M}(m)=T(m)$ é um isomorfismo sobre sua imagem.

Seja $S: T(M) \rightarrow M$ a inversa contínua de $T_{M}$. Então para $m \in M$ temos que $\left(I_{X}-S T\right)(m)=m-S T(m)=m-S T_{M}(m)=m-m=0$.

Portanto $M \subset N\left(I_{X}-S T\right)$. Assim, $N\left(I_{X}-S T\right)$ é de dimensão infinita e então pelo Lema 167 temos que $T \in L(X)-\operatorname{In}(X)$. Deste modo, provamos que $L(X)-\left(\Omega_{+}(X) \cap\right.$ $\left.\Omega_{-}(X)\right) \subset L(X)-\operatorname{In}(X)$ e, deste modo, por (i), temos que

$$
\operatorname{Imp}(X)=\operatorname{In}(X) \subset \Omega_{+}(X) \cap \Omega_{-}(X) .
$$

$(i i) \Rightarrow(i v)$

Colocando $\mathcal{A}(X)=\operatorname{Imp}(X)$ na Proposição 185 obtemos que $\operatorname{Imp}(X, Y)=\operatorname{In}(X, Y)$, para qualquer espaço de Banach $Y$.

$($ iii $) \Rightarrow($ iv $)$.

Segue da Proposição 185 que $\operatorname{In}(Y, X)=\operatorname{Imp}(Y, X)$, para qualquer espaço de Banach $Y$. Então pela Proposição 184 temos que $\operatorname{Imp}(X, Y)=\operatorname{In}(X, Y)$.

$(i v) \Rightarrow(i)$.

Basta tomar $Y=X$.

Definição 187. Um espaço de Banach $X$ é dito sub-projetivo se para qualquer subespaço fechado $M$ de dimensão infinita de $X$ existe um subespaço fechado $N \subset M$ de dimensão infinita tal que $N$ é complementado em $X$. 
Definição 188. Um espaço de Banach $X$ é dito super-projetivo se para qualquer subespaço fechado $M$ de codimensão infinita em $X$ existe um subespaço fechado $N \supset M$ de codimensão infinita em $X$ tal que $N$ é complementado em $X$.

Proposição 189. Sejam $X$ e $Y$ espaços de Banach. Suponhamos que um dos espaços de Banach $X$ ou $Y$ é sub-projetivo ou super-projetivo. Então $\operatorname{In}(X, Y)=\operatorname{Imp}(X, Y)$.

Prova. Caso 1) $Y$ é super-projetivo. Mostraremos que $L(X, Y)-\operatorname{In}(X, Y) \subset L(X, Y)-$ $\operatorname{Im} p(X, Y)$, ou equivalentemente, que $\operatorname{Imp}(X, Y) \subset \operatorname{In}(X, Y)$. Seja $T \in L(X, Y)-$ In $(X, Y)$. Então existe um operador $S \in L(Y, X)$ tal que $M=\overline{R\left(I_{Y}-T S\right)}$ é de codimensão infinita em $Y$. Como $Y$ é super-projetivo então existe um subespaço $N$ complementado em $Y, \operatorname{com} M \subset N$, tal que $\operatorname{codim}(N)=\infty$ em $Y$. Seja $P$ uma projeção tal que $N(P)=N$. Temos que $\operatorname{dim}(R(P))=\infty$, pois a imagem $R(P)$ de $P$ é o complemento algébrico do subespaço de codimensão infinita $N$. Além disso temos que $P\left(I_{Y}-T S\right)=0$, implicando que $(P T S)_{\left.\right|_{R(P)}}=I_{\left.Y\right|_{R(P)}}$ e portanto $(P T S)_{\left.\right|_{R(P)}}$ é um isomorfismo. Então $P T S \notin \operatorname{Imp}(Y)$, implicando, pela Proposição 176 , que $T \notin \operatorname{Imp}(X, Y)$.

Caso 2) $X$ é sub-projetivo. Seja $T \in L(X, Y)-\operatorname{In}(X, Y)$. Então existe um operador $S \in L(Y, X)$ tal que $M=N\left(I_{X}-S T\right)$ é de dimensão infinita. Como $X$ é sub-projetivo então existe um subespaço $N$ complementado em $X, \operatorname{com} N \subset M$, tal que $\operatorname{dim}(N)=\infty$. Desde que $N \subset M$ temos que $I_{\left.X\right|_{N}}-(S T)_{\left.\right|_{N}}=0$, implicando que $(S T)_{\left.\right|_{N}}=I_{\left.X\right|_{N}}$. Isto implica que $T(N)$ é um subespaço fechado, pois como $S \in L(Y, X)$ e $S(T(N))=N$ é fechado então $S^{-1}(N)=T(N)$ é um subespaço fechado. Além disso, $S J_{T(N)}$ é um isomorfismo e $S(T(N))=N$ é complementado em $X$. Segue do Lema 177 que o subespaço $T(N)$ é complementado em $Y$. Como $T J_{N}^{X}$ é um isomorfismo então $T \notin \operatorname{Imp}(X, Y)$.

Caso 3) $X$ é super-projetivo. Segue do caso 1 que $\operatorname{In}(Y, X)=\operatorname{Imp}(Y, X)$. Então pela Proposição 184 temos que $\operatorname{In}(X, Y)=\operatorname{Imp}(X, Y)$.

Caso 4) $Y$ é sub-projetivo. Pelo caso 2 temos que $\operatorname{In}(Y, X)=\operatorname{Imp}(Y, X)$, implicando pela Proposição 184 que $\operatorname{In}(X, Y)=\operatorname{Imp}(X, Y)$.

Proposição 190. Sejam $X$ e $Y$ espaços de Banach. Se $Y$ é sub-projetivo então $\operatorname{Imp}(X, Y)=$ $S S(X, Y)$.

Prova. Seja $T \in L(X, Y)-S S(X, Y)$. Então existe um subespaço fechado $M$ de $X$, $\operatorname{com} \operatorname{dim}(M)=\infty$, tal que $T J_{M}^{X}$ é um isomorfismo. Desde que $Y$ é sub-projetivo então existe um subespaço de dimensão infinita. $N$ de $T(M)$ que é complementado em $Y$. Seja. 
$A=\left(T_{\left.\right|_{M}}\right)^{-1}(N)$. Temos que $A$ é um subespaço fechado de dimensão infinita de $X$ e $A \subset M$. Como a restrição $T_{\left.\right|_{M}}$, de $T$ a $M$, é um isomorfismo então, em particular, $T J_{A}^{X}$ também é um isomorfismo. Portanto, desde que $T(A)=N$ é complementado então $T \notin \operatorname{Imp}(X, Y) . A \operatorname{ssim}, \operatorname{Imp}(X, Y) \subset S S(X, Y)$.

\subsection{Exemplo de um operador improjetivo que não é inessencial}

Nessa seção apresentaremos um exemplo, devido a González, M. e Aiena, P. [2], de um operador improjetivo que não é inessencial. Na descrição do exemplo os autores utilizam um espaço de Banach indecomponível construído por Gowers, W.T. e Maurey, B. Apresentaremos algumas definições e resultados preliminares para a apresentação de tal exemplo.

Definição 191. Sejam $X$ e $Y$ espaços de Banach. Definimos

$$
\begin{aligned}
& \Phi_{l}(X, Y)=\left\{T \in \Phi_{+}(X, Y): R(T) \text { é complementado }\right\} \\
& \Phi_{r}(X, Y)=\left\{T \in \Phi_{-}(X, Y): N(T) \text { é complementado }\right\}
\end{aligned}
$$

Lembremos que um espaço de Banach $X$ é dito decomponível se ele contém um par de subespaços fechados de dimensão infinita $M, N$ tais que $X=M \oplus N$. Caso contrário, isto é, se não existem tais subespaços em $X$ então $X$ é dito indecomponível.

Proposição 192. Para um espaço de Banach $Y$ as seguintes afirmações são equivalentes:

(i) $Y$ é indecomponivel;

(ii) $L(Y, Z)=\Phi_{l}(Y, Z) \cup \operatorname{Imp}(Y, Z)$, para qualquer espaço de Banach $Z$;

(iii) $L(X, Y)=\Phi_{r}(X, Y) \cup \operatorname{Imp}(X, Y)$, para qualquer espaço de Banach $X$;

(iv) $L(Y)=\Phi(Y) \cup \operatorname{Imp}(Y)$.

Prova. $(i) \Rightarrow($ ii $)$

Seja $T \in L(Y, Z)-\operatorname{Imp}(Y, Z)$. Então existe um subespaço fechado de dimensão infinita. $M$ de $Y$ tal que $T J_{M}^{X}$ é um isomorfismo e $T(M)$ é complementado em $Z$, digamos que $Z=T(M) \oplus N$, onde $N$ é o complemento fechado de $T(M)$. Segue do Lema 177 que $M$ é complementado em $Y$ e $Y=M \oplus T^{-1}(N)$. Desde que $Y$ é indecomponível e $\operatorname{dim}(M)=\infty$ 
então $\operatorname{codim}(M)<\infty$ em $Y$, implicando que $\operatorname{dim}\left(T^{-1}(N)\right)<\infty$. Como $T J_{M}^{X}$ é um isomorfismo então $N(T) \cap M=\{0\}$, implicando que $N(T) \subset T^{-1}(N)$. Portanto,

$$
\operatorname{dim}(N(T))<\infty
$$

Então existe um subespaço de dimensão finita $N_{o}$ tal que $R(T)=T(M) \oplus N_{0}$. Logo,

$$
R(T) \text { é complementado em } \mathrm{Z} \text {. }
$$

Segue de (5.13) e (5.14) que $T \in \Phi_{l}(Y, Z)$.

(i) $\Rightarrow($ iii $)$

Seja $T \in L(X, Y)-\operatorname{Imp}(X, Y)$. Segue da Proposição 178 que existe um subespaço fechado de codimensão infinita $N$ de $Y$ tal que $Q_{N} T$ é sobrejetor e $T^{-1}(N)$ é complementado em $X$. Digamos que $X=T^{-1}(N) \oplus M$, onde $M$ é o complemento fechado de $T^{-1}(N)$ em $X$. Então $N$ é complementado em $Y$ e $Y=N \oplus T(M)$. Desde que $\operatorname{dim}(T(M))=\infty$ e $Y$ é indecomponível, segue que $\operatorname{dim}(N)<\infty$, implicando que $\operatorname{codim}(R(T))<\infty$ em $Y$. Além disso, como $R(T)=T(M) \oplus N_{o}$, onde $N_{o}$ é um subespaço de $N$, então $R(T)$ é um subespaço fechado. Portanto,

$$
T \in \Phi_{-}(X, Y)
$$

Temos que $T^{-1}(N)=N(T) \oplus M_{o}$, onde $M_{o}$ é um subespaço de dimensão finita. Isto implica que

$$
N(T) \text { é complementado em X. }
$$

Segue de (5.15) e (5.16) que $T \in \Phi_{r}(X, Y)$. $(i i) \Rightarrow(i v)$.

Seja $T \in L(Y)-\operatorname{Imp}(Y)$. Então, por hipótese, $T \in \Phi_{l}(Y)$ e $\operatorname{dim}(R(T))=\infty$. Como $T \in \Phi_{l}(Y)$ então $\operatorname{dim}(N(T))<\infty$ e $R(T)$ é complementado em $Y$. Isto implica que $Y=R(T) \oplus N$, onde $N$ é o complemento fechado de $R(T)$. Seja $S: Y \rightarrow R(T)$ uma projeção sobre $R(T)$. Então $N=N(S)$. Deste modo, podemos escrever

$$
Y=R(T) \oplus N(S)
$$

Seja $y \in N(T)$. Então $T(y)=0$, implicando que $S T(y)=S(0)=0$. Isto implica que $y \in N(S T)$ e portanto, $N(T) \subset N(S T)$. Por outro lado, se $y \in N(S T)$ então $S T(y)=0$, implicando que $T(y) \in N(S)$. Logo, $T(y)=0$, pois $T(y) \in R(T)$ e $R(T) \cap N=\{0\}$. Então segue que $y \in N(T)$ e portanto, $N(S T) \subset N(T)$. Deste modo, $N(S T)=N(T)$. 
Temos também que $S J_{R(T)}^{Y}$ é um isomorfismo. Com efeito, seja $y \in R(T)$. Se $S(y)=0$ então $y \in N(S)$. Como $R(T) \cap N(S)=\{0\}$ então $y=0$ implicando que $S J_{R(T)}^{Y}$ é injetor. Então pelo Teorema da aplicação aberta $S J_{R(T)}^{Y}$ é um isomorfismo. Como $S(R(T))=R(T)$ é complementado então $S \notin \operatorname{Imp}(Y)$, implicando por (b) que $S \in \Phi_{l}(Y)$ e isto implica. que $\operatorname{dim}\left(N\left(S^{\prime}\right)\right)<\infty$. Isso por sua vez implica, por (5.17), que $\operatorname{codim}(R(T))<\infty$ em $Y$. Desde que, por hipótese, $\operatorname{dim}(N(T))<\infty$ então $T \in \Phi(Y)$. $($ iii $) \Rightarrow(i v)$.

Seja $T \in L(Y)-\operatorname{Imp}(Y)$. Segue da hipótese que $T \in \Phi_{r}(Y)$ e $\operatorname{codim}(N(T))=\infty$ em $Y$. Então segue que $N(T)$ é complementado, implicando que existe uma projeção $S \in L(Y)$, $\operatorname{com} N(S)=N(T)$, tal que

$$
Y=N(T) \oplus R(S)
$$

Temos que $R(T S) \subset R(T)$. Além disso, se $y \in R(T)$ então existe $y^{\prime} \in Y$ tal que $y=T\left(y^{\prime}\right)$. Como $Y=N(T) \oplus R(S)$ então existe $u \in N(T)$ e $v \in R(S)$ tais que $y^{\prime}=u+v$. Isto implica que $y=T(v)$ e então existe $x \in Y$ tal que $v=S(x)$. Deste modo, temos que $y=T(v)=T S(x)$, implicando que $y \in R(T S)$. Logo, $R(T S)=R(T)$, implicando que TS é uma projeção, pois $(T S)^{2}=T S T S=(T S) T=T=T S$. Segue de (5.18) que o operador $Q_{N(T)} S$ é sobrejetor, onde $Q_{N}: Y \rightarrow Y / N$ é a aplicação quociente canônica. Como $x \in S^{-1}(N(T))$ então $S(x) \in N(T)$, implicando que $T S(x)=0$, que por sua vez implica que $x \in N(T S)$ e portanto $S^{-1}(N(T))=N(T S)$ é complementado pois TS é uma projeção. Assim, pela Proposição 178, temos que se $S \notin \operatorname{Imp}(Y)$ então por (iii) temos que $S \in \Phi_{r}(Y)$, implicando que $S \in \Phi_{-}(Y)$. Isto implica que $\operatorname{codim}(R(S))<\infty$ em $Y$. Segue por (5.18) que $\operatorname{dim}(N(T))<\infty$. Portanto, $T \in \Phi(Y)$. (iv) $\Rightarrow(i)$.

Suponhamos que $Y$ é decomponível. Então existe uma projeção $P \in L(Y)$ tal que $N(P)$ e $R(P)$ são ambos de dimensão infinita. Então $P \notin \Phi(Y) \cup \operatorname{Imp}(Y)$.

Proposição 193. Seja $X$ um espaço de Banach. Suponhamos que ou todo subespaço fechado de $X$ é indecomponivel ou todo quociente de $X$ é indecomponivel. Então $L(X)=$ $\Phi(X) \cup \operatorname{In}(X)$.

Prova. Como $S S(X) \subset \operatorname{In}(X)$ e $S C(X) \subset \operatorname{In}(X)$ então, pela Proposição 62 , temos que

$$
L(X)=\Phi_{+}(X) \cup \Phi_{-}(X) \cup \operatorname{In}(X) .
$$


Suponhamos que exista $T \in L(X)-(\Phi(X) \cup \operatorname{In}(X))$. Então por (5.19) temos que $T \in \Phi_{+}(X) \cup \Phi_{-}(X), \operatorname{dim}(X)=\infty$ e $i(T)=\{ \pm \infty\}$.

A Proposição 102 mostra que o conjunto de todos os operadores em $\Phi_{+}(X) \cup \Phi_{-}(X)$ com índice igual a um valor fixado é aberto. Se $|t|>\|T\|$ então $\|T / t\|<1$, implicando que o operador $I-\frac{T}{t}$ é inversível e portanto o operador $t\left(I-\frac{T}{t}\right)$ é inversível. Logo, o operador $t I_{X}-T$ é inversível para $|t|>\|T\|$. Então existem $c, d$ com $c<0<d$ tais que $c I_{X}-T$ e $d I_{X}-T \notin \Phi_{+}(X) \cup \Phi_{-}(X)$. Isto implica, por (5.19), que $c I_{X}-T$ e $d I_{X}-T \in$ $\operatorname{In}(X)$. Como $\operatorname{In}(X)$ é um subespaço de $L(X)$ então $(c-d) I_{X} \in \operatorname{In}(X) \subset \operatorname{Imp}(X)$, implicando que $\operatorname{dim}(X)<\infty$, que é uma contradição.

Proposição 194. Seja $X$ um espaço de Banach. Suponhamos que $L(X)=\Phi(X) \cup \operatorname{In}(X)$. Então $\operatorname{Imp}(X, Y)=\operatorname{In}(X, Y)$ para qualquer espaço de Banach $Y$.

Prova. O resultado é imediato se $\operatorname{In}(X, Y)=L(X, Y)$. Então podemos assumir que existe $T \in L(X, Y)-\operatorname{In}(X, Y)$. Mostraremos que $T \in L(X, Y)-\operatorname{Imp}(X, Y)$. De fato, segue da Proposição 165 que existe $S \in L(Y, X)$ tal que $\operatorname{dim}\left(N\left(I_{X}-S T\right)\right)=\infty$. Logo, $I_{X}-S T \notin$ $\Phi(X)$, implicando que $I_{X}-S T \in I n(X)$. Portanto temos que $S T=I_{X}-\left(I_{X}-S T\right) \in \Phi(X)$ e isto implica que $S T \in \Phi_{+}(X) \cap \Phi_{-}(X)$.

Como $N(T) \subset N(S T)$ então $\operatorname{dim}(N(T))<\infty$. Logo, temos que $T \in \Phi_{+}(X, Y)$. Além disso, a imagem $R(T)$ de $T$ é um subespaço complementado em $Y$ e, portanto, $T \in \Phi_{l}(X, Y)$.

Desde que $X=N(T) \oplus M$, onde $M$ é o complemento fechado de $N(T)$ em $X, T J_{M}^{X}$ é um isomorfismo e $T(M)=R(T)$ é complementado então segue que $T \notin \operatorname{Im} p(X, Y)$.

Teorema 195. Existe um espaço de Banach complexo $Z$ com as seguintes propriedades:

(a) $\operatorname{In}(Z) \neq \operatorname{Imp}(Z)$;

(b) Existe $T \in \operatorname{Imp}(Z)$ que não é um operador de Riesz;

(c) $\operatorname{Imp}(Z)$ não é um subespaço de $L(Z)$.

Prova. Consideremos o espaço de Banach complexo $Z$ introduzido por Gowers, W.T. e Maurey, B. (veja Teorema 59). Os autores mostraram que tal espaço $Z$ possui uma base de Schauder $\left\{z_{n}\right\}$ e que o operador deslocamento à direita $S: Z \rightarrow Z$, definido por $S\left(a_{1}, a_{2}, \ldots\right)=\left(0, a_{1}, a_{2}, \ldots\right)$, é uma isometria sobre $Z$. Além disso, $Z$ é um espaço de Banach indecomponível. Então, pela Proposição 192, temos que 


$$
L(Z)=\Phi(Z) \cup \operatorname{Imp}(Z)
$$

Seja $\lambda \in \mathbb{C}$. Para $|\lambda|>1$ temos, pela Proposição 52 , que o operador $I_{Z}-\frac{S}{\lambda}$ é inversível pois $\|S\|=1$. Então o operador $\lambda I_{Z}-S=\lambda\left(I_{Z}-\frac{S}{\lambda}\right)$ é inversível para $|\lambda|>1$. Portanto o operador $\lambda I_{Z}-S$ é um operador de Fredholm com $i\left(\lambda I_{Z}-S\right)=0$ para $|\lambda|>1$.

Por outro lado, para $|\lambda|<1$, dado $x=\left(a_{1}, a_{2}, a_{3}, \ldots\right) \in Z-[(1,0,0, \ldots)]$ temos que $\left(\lambda I_{Z}-S\right)\left(\frac{a_{1}}{\lambda}, \frac{\lambda a_{2}+a_{1}}{\lambda^{2}}, \frac{\lambda^{2} a_{3}+\lambda a_{2}+a_{1}}{\lambda^{3}}, \ldots, \frac{\sum_{i=1}^{k} \lambda^{i-1} a_{i}}{\lambda^{k}}, \ldots\right)=\left(a_{1}, a_{2}, a_{3}, \ldots\right), \lambda \neq 0$ (observemos que $(x, 0,0, \ldots) \notin R\left(\lambda I_{Z}-S\right)$ se $\left.x \neq 0\right)$. Se $\lambda=0$ então $\lambda I_{Z}-S=-S$ e $Z=A \oplus S(Z)$, onde $A=[(1,0,0,0, \ldots)]$. Portanto, $\beta\left(\lambda I_{Z}-S\right)=1$ para $|\lambda|<1$. Além disso,

$$
\begin{gathered}
N\left(\lambda I_{Z}-S\right)=\left\{\left(a_{1}, a_{2}, a_{3}, \ldots\right) \in Z:\left(\lambda a_{1}, \lambda a_{2}-a_{1}, \lambda a_{3}-a_{2}, \ldots\right)=(0,0,0, \ldots)\right\}= \\
=\{(0,0,0, \ldots)\} .
\end{gathered}
$$

Logo, $\alpha\left(\lambda I_{Z}-S\right)=0,|\lambda|<1$. Assim, $i\left(\lambda I_{Z}-S\right)=-1$ para $|\lambda|<1$. A função $\varphi: \mathbb{C} \rightarrow \Phi_{+}(Z) \cup \Phi_{-}(Z)$, definida por $\varphi(\lambda)=\lambda I_{Z}-S$ e a função índice de um operador $i: \Phi_{+}(Z) \cup \Phi_{-}(Z) \rightarrow \mathbb{Z}$ são funções contínuas. Então a aplicação composta $i$ o $\varphi$ é também contínua. Como

$$
i\left(\lambda I_{Z}-S\right)=\left\{\begin{array}{lll}
0, & \text { se } & |\lambda|>1 \\
1, & \text { se } & |\lambda|<1
\end{array}\right.
$$

então segue, pela Proposição 102, que $\lambda I_{Z}-S \notin \Phi_{+}(Z) \cup \Phi_{-}(Z)$ para $|\lambda|=1$. Em particular, $\lambda I_{Z}-S \notin \Phi(Z)$ para $|\lambda|=1$. Portanto, para $|\lambda|=1$, temos que $\lambda I_{Z}-S \in$ $\operatorname{Imp}(Z)$. Mas $\lambda I_{Z}-S$ não é um operador de Riesz para $|\lambda|=1$. Para mostrar isto, devemos mostrar que para $|\lambda|=1$ existe $\beta \neq 0$ tal que $\left(\frac{1-\beta \lambda}{\beta}\right) I_{Z}-S \notin \Phi(Z)$. Desejamos encontrar $\beta \neq 0$ tal que $\left|\frac{1-\beta \lambda}{\beta}\right|=1$, ou ainda, $|1-\beta \lambda|=|\beta|$. Em particular, para $\lambda=1$ basta tomar $\beta=\frac{1}{2}$ e para $\lambda=-1$ basta tomar $\beta=-\frac{1}{2}$. Como todo operador inessencial é um operador de Riesz então, em particular, $I_{Z}-S \in \operatorname{Imp}(Z)-\operatorname{In}(Z)$.

Por outro lado, $\left(I_{Z}-S\right)-\left(-I_{Z}-S\right)=2 I_{Z} \notin \operatorname{Imp}(Z)$, desde que $Z$ é de dimensão infinita. Então $\operatorname{Imp}(Z)$ não é um subespaço de $L(Z)$.

Esse teorema responde negativamente as questões 1 e 2 apresentadas na seção anterior. 
Teorema 196. Existe um par de espaços de Banach $Z, Y$ para os quais $\operatorname{In}(Z, Y) \neq$ $\operatorname{Imp}(Z, Y)=L(Z, Y)$.

Prova. Seja $Z$ o espaço de Banach considerado na prova do Teorema 195. Consideremos o operador $T=I_{Z}-S \in L(Z)$.

Pela Proposição 102 temos que $T \notin \Phi_{-}(Z)$. Deste modo, podemos encontrar um operador compacto $K \in L(Z)$ tal que $\overline{R(T-K)}$ é de codimensão infinita em $Z$ (ver Proposição 106). Tomemos $Y=\overline{R(T-K)}$ e consideremos o operador $U \in L(Z, Y)$ definido por $U(z)=T(z)-K(z), \forall z \in Z$. Como $T-K=J_{\overline{R(T-K)}}$ não é inessencial então $U$ não é inessencial. Além disso, todo subespaço complementado de dimensão infinita de $Z$ é isomorfo a $Z$ (ver Teorema 59(ii)), mas $Z$ não é isomorfo a qualquer de seus subespaços fechados de codimensão infinita (ver Teorema 59(iii)). Então $\operatorname{Imp}(Z, Y)=L(Z, Y)$, pela Proposição 180.

Conforme González, M. e Aiena, P. observaram em [2], $\operatorname{Imp}(Z, Y)=L(Z, Y)$ se, e somente se, $Z$ e $Y$ são projeção totalmente incomparáveis (isto é, se não existe em $Z$ um subespaço complementado de dimensão infinita isomorfo a um subespaço complementado de $Y$ ), conforme vimos na Proposição 180. O exemplo no Teorema 196 permite-nos mostrar que $\operatorname{In}(Z, Y) \neq L(Z, Y)$ não implica que $Z$ possui um subespaço complementado de dimensão infinita isomorfo a um subespaço complementado de $Y$, o que responde de modo negativo a seguinte conjectura de González, M.:

Conjectura. ([10], página 625) Dois espaços de Banach $X$ e $Y$ são projeção totalmente incomparáveis se, e somente se, $\operatorname{In}(X, Y)=L(X, Y)$.

Além disso, o fato de $\operatorname{Imp}(Z, Y)$ ser um subespaço de $L(Y, Z)$ não implica que a classe $\operatorname{Imp}(Z, Y)$ coincide com a classe $\operatorname{In}(Z, Y)$. Logo, a resposta para a Questão 3 apresentada. na seção anterior é negativa. 
Capítulo 6

\section{Subespaços complementados na soma de espaços de Banach essencialmente incomparáveis}

O objetivo desse capítulo é estudar o seguinte resultado obtido por González, M. [10]:

Sejam $Y$ e $Z$ espaços de Banach tais que $\operatorname{In}(Y, Z)=L(Y, Z)$. Então, para todo subespaço complementado $M$ em $Y \oplus Z$ existe um isomorfismo bijetor $U \in L(Y \oplus Z)$ e subespaços $Y_{o}$ complementado em $Y$ e $Z_{o}$ complementado em $Z$ tais que $U(M)=Y_{o} \oplus Z_{o}$.

A prova é similar à prova do Teorema 149 requerendo a utilização do conceito de $\Phi$-ideais (veja Definição 98), que é necessário para algumas adaptações na prova.

Definição 197. Seja $T \in L(X)$, onde $X$ é um espaço de Banach. Definimos $\rho_{e}(T)=$ $\{\lambda \in \mathbb{C}: i(\lambda I-T)=0\}$. O conjunto $\sigma_{e}=\mathbb{C}-\rho_{e}(T)$ é chamado o espectro essencial de $T$.

Lema 198. Seja $P=S+Q$, onde $Q \in L(X), S \in \operatorname{In}(X)$ e $X$ é um espaço de Banach. Então $z I-P \in \Phi(X)$ se, e somente se, $z I-Q \in \Phi(X)$.

Prova. Suponhamos que $z I-P \in \Phi(X)$. Como $S \in \operatorname{In}(X)$ e $\operatorname{In}(X)$ é um $\Phi$-ideal então segue da. Proposição 116 que $z I-P+S \in \Phi(X)$ e $i(z I-P+S)=i(z I-P)$. Como $-P+S=-Q$ então segue que $z I-Q \in \Phi(X)$.

Reciprocamente, se $z I-Q \in \Phi(X)$ então $z I-Q-S \in \Phi(X)$ e $i(z I-Q-S)=i(z I-Q)$, isto é, $z I-P \in \Phi(X)$.

Logo, os operadores $P$ e $Q$ possuem o mesmo espectro essencial. 
Subespaços complementados na soma de espaços de Banach essencialmente 104 incomparáveis

Lema 199. Seja $X$ um espaço de Banach complexo e seja $P$ uma projeção em $X$. Seja $S: X \rightarrow X$ um operador inessencial e seja $Q=P+S$. Então o espectro $\sigma(Q)$ de $Q$ é enumerável e seus únicos possiveis pontos limites são 0 e 1 .

Prova. Seja $\mathbb{C}$ o plano complexo.

(i) $Q-\lambda I$ é um operador de Fredholm de índice $0, \forall \lambda \in \mathbb{C}-\{0,1\}$.

Temos que

$$
Q-\lambda I=(P+S)-\lambda I=(P+S)-\lambda I=(P-\lambda I)+S
$$

O operador $P-\lambda I$ é contínuo e possui imagem fechada, pois $P$ é uma projeção. Se o operador linear $P-\lambda I$ é uma projeção então não necessariamente temos $\alpha(P-\lambda I)<\infty$. No entanto, $P-\lambda I$ é uma projeção se, e somente se,

$$
P-\lambda I=(P-\lambda I)^{2}=(P-\lambda I)(P-\lambda I)=P^{2}-2 \lambda P+\lambda^{2} P=P-2 \lambda P+\lambda^{2} P,
$$

que implica $\left(\lambda^{2}-\lambda\right) x=0, \forall x \neq 0$ em $R(P)$ e portanto $\lambda(\lambda-1)=0$, implicando que $\lambda=0$ ou $\lambda=1$.

Portanto o operador linear $P-\lambda I$ é uma projeção se, e somente se, $\lambda=0$ ou $\lambda=1$. Se $\lambda \in \mathbb{C}-\{0,1\}$ então para $x \in N(P-\lambda I)$ temos que $(P-\lambda I)(x)=0$ implicando que $P(x)=\lambda x$ e então $P^{2}(x)=\lambda P(x)$, isto é, $P(x)=\lambda(\lambda x)$ e isto implica que $\lambda x=\lambda^{2} x$. Deste modo, $\left(\lambda^{2}-\lambda\right) x=0$.

Como $\lambda \in \mathbb{C}-\{0,1\}$ então $x=0$. Logo, $N(P-\lambda I)=\{0\}$ quando $\lambda \in \mathbb{C}-\{0,1\}$. Conseqüentemente temos que

$$
\alpha(P-\lambda I)=0, \forall \lambda \in \mathbb{C}-\{0,1\} .
$$

Além disso, $\operatorname{codim}(R(P-\lambda I))=0$ em $X$, ou equivalentemente, o operador $P-\lambda I$ é sobre $X$, para $\lambda \in \mathbb{C}-\{0,1\}$. Com efeito, como $P: X \rightarrow X$ é uma projeção então $P^{*}: X^{*} \rightarrow X^{*}$ também o é, pois

$\left(P^{*}\right)^{2} x^{*}(x)=P^{*}\left(P^{*} x^{*}(x)\right)=P^{*} x^{*}(P(x))=x^{*} P^{2}(x)=x^{*} P(x)=P^{*} x^{*}(x), \forall x \in X$.

Então, pelo que provamos no parágrafo anterior, temos que

$$
N\left(P^{*}-\lambda I^{*}\right)=0, \forall \lambda \in \mathbb{C}-\{0,1\}
$$

e daí,

$$
\alpha\left(P^{*}-\lambda I^{*}\right)=0, \forall \lambda \in \mathbb{C}-\{0,1\} .
$$


Segue pela. Proposição 77 e por (6.3) que

$$
\beta(P-\lambda I)=\alpha\left((P-\lambda I)^{*}\right)=\alpha\left(P^{*}-\lambda I^{*}\right)=0, \forall \lambda \in \mathbb{C}-\{0,1\}
$$

De (6.2) e (6.3) temos que

$$
i(P-\lambda I)=0, \forall \lambda \in \mathbb{C}-\{0,1\} .
$$

Pela Proposição 116 segue que

$$
i(Q-\lambda I)=i((P-\lambda I)+S)=i(P-\lambda I)=0
$$

(ii) $\sigma(Q)$ é enumerável.

O conjunto $\mathbb{C}-\{0,1\}$ é conexo (por caminhos) de acordo com a Proposição 15. Então quaisquer dois pontos de $\mathbb{C}-\{0,1\}$ estão contidos num mesmo subconjunto conexo de $\mathbb{C}-\{0,1\}$ e, então, numa mesma componente conexa de $\mathbb{C}-\{0,1\}$. Então, pela Proposição 107 esses dois pontos arbitrários podem ser conectados por uma quantidade finita de discos abertos que interceptam-se e onde $\alpha(Q-\lambda I)$ é constante, exceto possivelmente nos centros dos discos.

Portanto $\alpha(Q-\lambda I)$ é constante sobre $C-\{0,1\}$, exceto possivelmente num conjunto de pontos isolados.

Vimos na. Proposição 128 que $\sigma(Q) \subset\{\lambda:|\lambda| \leq\|Q\|\}$. Então $Q-\lambda I$ é inversível para. $|\lambda|>\|Q\|$. Em tais pontos o operador $Q-\lambda I$ é injetor, implicando que $N(Q-\lambda I)=\{0\}$. Logo $\alpha(Q-\lambda I)=0$, exceto possivelmente sobre um conjunto de pontos isolados. Então

$$
0=i(Q-\lambda I)=\alpha(Q-\lambda I)-\beta(Q-\lambda I)=\beta(Q-\lambda I)
$$

exceto possivelmente em um conjunto de pontos isolados.

Deste modo, o espectro $\sigma(Q)$ de $Q$ está contido em um conjunto de pontos isolados, implicando, pela Proposição 144, que $\sigma(Q)$ é enumerável.

Lembremos que dado um espaço vetorial $X$ e um subespaço vetorial $M$ de $X$, definimos a codimensão de $M$ em $X$ como a dimensão do espaço quociente $X / M$. Se a dimensão de $X / M$ é finita então dizemos que $M$ é de codimensão finita em $X$. A proposição a seguir é um resultado elementar de Álgebra Linear que apresenta uma definição equivalente para subespaços de codimensão finita. Omitiremos a demonstração por tratar-se de um resultado elementar. 
Subespaços complementados na soma de espaços de Banach essencialmente 106

incomparáveis

Proposição 200. Sejam $X$ um espaço vetorial, $Y$ um subespaço de $X$ e $n \in \mathbb{N}$. Então $\operatorname{dim}(X / Y)=n$ se, e somente se, existem vetores $x_{1}, \ldots, x_{n} \in X$ linearmente independentes tal que $X=Y \oplus\left[x_{1}, \ldots, x_{n}\right]$.

Lema 201. Sejam $Y$ e $Z$ espaços de Banach. Se $Y_{1}$ tem codimensão finita em $Y$ e $Z_{1}$ tem codimensão finita em $Z$, então $Y_{1} \oplus Z_{1}$ tem codimensão finita em $Y \oplus Z$.

Prova. Sejam $y_{1}, \ldots, y_{n}$ em $Y$ vetores linearmente independentes tais que

$$
Y=\left[Y_{1} \cup\left\{y_{1}, \ldots, y_{n}\right\}\right]
$$

e sejam $z_{1}, \ldots, z_{m}$ em $Z$ vetores linearmente independentes tais que

$$
Z=\left[Z_{1} \cup\left\{z_{1}, \ldots, z_{m}\right\}\right]
$$

Temos por (6.6) e (6.7) que

$$
Y \oplus Z=\left[Y_{1} \cup\left\{y_{1}, \ldots, y_{n}\right\}\right] \oplus\left[Z_{1} \cup\left\{z_{1}, \ldots, z_{m}\right\}\right]
$$

Portanto, se $(y, z) \in Y \oplus Z$ então $y=\alpha y^{\prime}+\alpha_{1} y_{1}+\cdots+\alpha_{n} y_{n}$, onde $\alpha, \alpha_{1}, \ldots, \alpha_{n} \in$ $\mathbb{C}, y^{\prime} \in Y_{1}$ e $z=\beta z^{\prime}+\beta_{1} z_{1}+\cdots+\beta_{m} z_{m}$, onde $\beta, \beta_{1}, \ldots, \beta_{m} \in \mathbb{C}, z^{\prime} \in Z_{1}$. Então

$$
\begin{aligned}
(y, z) & =\left(\alpha y^{\prime}+\alpha_{1} y_{1}+\cdots+\alpha_{n} y_{n}, \beta z^{\prime}+\beta_{1} z_{1}+\cdots+\beta_{m} z_{m}\right) \\
& =\left(\alpha y^{\prime}, \beta z^{\prime}\right)+\left(\alpha_{1} y_{1}+\cdots+\alpha_{n} y_{n}, \beta_{1} z_{1}+\cdots+\beta_{m} z_{m}\right)
\end{aligned}
$$

Portanto, $Y_{1} \oplus Z_{1}$ tem codimensão finita.

Lema 202. Seja $M$ um subespaço de codimensão infinita de um espaço de Banach X. Então qualquer subespaço $M^{\prime}$ de $M$ tem codimensão infinita em $X$.

Prova. Suponhamos que $M^{\prime}$ possui codimensão finita em $X$. Então existe uma quantidade finita de vetores $x_{1}, \ldots, x_{k}$ em $X$ linearmente independentes tais que $X=\left[M^{\prime} \cup\right.$ $\left.\left\{x_{1}, \ldots, x_{k}\right\}\right]$. Então $M$ também é de codimensão finita em $X$, o que contradiz a hipótese.

Teorema 203. Sejam $Y$ e $Z$ dois espaços de Banach tais que $\operatorname{In}(Y, Z)=L(Y, Z)$. Seja $P$ uma projeção de $Y \oplus Z$ sobre um subespaço $M$. Então existe um isomorfismo $U$ de $Y \oplus Z$ sobre $Y \oplus Z$ e subespaços complementados $Y_{\circ}$ de $Y$ e $Z_{\circ}$ de $Z$ tais que $U(M)=Y_{\circ} \oplus Z_{\circ}$. 
Prova. Faremos a prova para o caso em que $Y$ e $Z$ são espaços de Banach sobre o conjunto dos números complexos. O caso real segue de modo análogo pelas considerações feitas na prova do Teorema 149 utilizando um processo de complexificação de espaços de Banach. Vamos assumir que

$M$ é de dimensão infinita e de codimensão infinita,

Se $\operatorname{dim}(M)<\infty$ então podemos encontrar subespaços de dimensão finita (e portanto complementados) $Y_{\circ}$ de $Y$ e $Z_{\circ}$ de $Z$ tais que $\operatorname{dim}\left(Y_{\circ} \oplus Z_{\circ}\right)=\operatorname{dim}(M)<\infty$ e o resultado segue imediatamente. Se $\operatorname{codim}(M)=n<\infty$ em $Y \oplus Z$ então como $\operatorname{dim}(M)=\infty$ (pelo que supomos anteriormente) podemos assumir que $\operatorname{dim}(Y)=\infty$, implicando a existência de um subespaço $Y_{\circ}$ de $Y$ tal que $Y=Y_{\circ} \oplus \mathbb{C}^{n}$. Como $\operatorname{codim}(M)=n$ em $Y \oplus Z$ então $\left(Y_{\circ} \oplus \mathbb{C}^{n}\right) \oplus Z=Y \oplus Z=M \oplus \mathbb{C}^{n}$. Desde que todos os hiperplanos (subespaços de codimensão 1) são isomorfos entre si, segue que $M=Y_{\circ} \oplus Z$, que é o resultado desejado.

Consideraremos os seguintes passos:

$1^{\underline{O})}$ A decomposição natural da projeção $P$ em operadores de $Y$ em $Y$, de $Y$ em $Z$, de $Z$ em $Y$ e de $Z$ em $Z$ e escrevemos $P$ em sua representação matricial em termos destes operadores.

$2^{\underline{Q}}$ ) Obteremos a partir de $P$ um operador inessencial $S$ que permitirá a construção de um operador $Q$ cujo espectro é enumerável e seus únicos pontos de acumulação são os números 0 e 1 .

$3^{\underline{O}}$ ) Consideraremos então uma outra projeção $P_{1}$ sobre $Y \oplus Z$.

$4^{\mathrm{O}}$ ) Obteremos a partir de $P_{1}$ um operador de Fredholm $V$ em $Y \oplus Z$ com índice 0 .

$5^{\underline{\mathrm{O}}}$ ) Então consideraremos um operador de posto finito $A \in L(Y \oplus Z)$.

$6^{\underline{0}}$ ) Construiremos a partir de $A$ um operador $k \in L(Y \oplus Z)$.

$7^{\circ}$ ) Finalmente obteremos o isomorfismo $U$ e os subespaços complementados $Y_{o}$ e $Z_{0}$.

1으 passo.) Escrevemos a projeção $P: Y \oplus Z \rightarrow M \subset Y \oplus Z$ do seguinte modo

$$
P(y, z)=\left(T_{1}(y, z), T_{2}(y, z)\right), \forall(y, z) \in Y \oplus Z,
$$

onde $T_{1}: Y \oplus Z \rightarrow Y$ e $T_{2}: Y \oplus Z \rightarrow Z$ são operadores lineares. Temos que

$$
P(y, z)=\left(T_{1}(y, z), T_{2}(y, z)\right)=\left(T_{1}\left(\pi_{1}(y)\right)+T_{1}\left(\pi_{2}(z)\right), T_{2}\left(\pi_{1}(y)\right)+T_{2}\left(\pi_{2}(z)\right)\right),
$$


Subespaços complementados na soma de espaços de Banach essencialmente 108 incomparáveis

onde $\pi_{1}: Y \rightarrow Y \oplus\{0\}$ e $\pi_{2}: Z \rightarrow\{0\} \oplus Z$ são operadores lineares definidos, respectivamente, por $\pi_{1}(y)=(y, 0), \forall y \in Y$ e $\pi_{2}(z)=(0, z), \forall z \in Z$. Logo,

$$
P(y, z)=\left(S_{1}(y)+S_{3}(z), S_{2}(y)+S_{4}(z)\right), \forall(y, z) \in Y \oplus Z
$$

onde $S_{1}=T_{1} \pi_{1}: Y \rightarrow Y, S_{2}=T_{2} \pi_{1}: Y \rightarrow Z, S_{3}=T_{1} \pi_{2}: Z \rightarrow Y$ e $S_{4}=T_{2} \pi_{2}: Z \rightarrow Z$, ou ainda, na representação matricial

$$
\dot{P}=\left(\begin{array}{ll}
S_{1} & S_{3} \\
S_{2} & S_{4}
\end{array}\right)
$$

$2^{\underline{o}}$ passo.) Segue da Proposição 166 que $S_{3} \in \operatorname{In}(Z, Y)$ implicando que

$$
S=\left(\begin{array}{cc}
0 & S_{3} \\
S_{2} & 0
\end{array}\right)
$$

é também um operador inessencial em $Y \oplus Z$. Seja $Q=P-S$. Segue do Lema 198 que os operadores $Q$ e $P$ possuem o mesmo espectro essencial, isto é, dado um número complexo $\lambda$ temos que $\lambda I-P \in \Phi(Y \oplus Z)$ se, e somente se, $\lambda I-Q \in \Phi(Y \oplus Z)$.

3 o passo.) Substituiremos o operador $Q$ por uma projeção $P_{1}$ possuindo uma representação matricial similar a de $Q$. Além disso, segue do Lema 199 que $\sigma(Q)$ é enumerável e os números 0 e 1 são os únicos pontos de acumulação de $\sigma(Q)$. Então existe uma curva $\Gamma$ simples, fechada, que não intercepta $\sigma(Q)$, possui os número $1 \mathrm{em}$ seu interior e os números 0 e 2 em seu exterior. Então definimos

$$
P_{1}=\frac{1}{2 \pi i} \int_{\Gamma} R_{Q}(\lambda) d \lambda
$$

onde $R_{Q}(\lambda)$ é o operador resolvente de $Q$. O operador $P_{1}$ está associado a função $g: \mathbb{C} \rightarrow \mathbb{C}$ definida por $g(\lambda)=1, \forall \lambda \in \mathbb{C}$, isto é, $P_{1}=g(Q)$. Pela Fórmula (3.22), da Observação 136 , temos que

$$
Q=\frac{1}{2 \pi i} \int_{\Gamma} \lambda R_{Q}(\lambda) d \lambda
$$

isto é, $Q$ está associado a função $f: \mathbb{C} \rightarrow \mathbb{C}$ definida por $f(\lambda)=\lambda, \forall \lambda \in \mathbb{C}$. Segue da Proposição 137-(ii) que

$$
P_{1}=P_{1} Q=g(Q) f(Q)=(g f) Q=(f g)(Q)=f(Q) g(Q)=Q P_{1} .
$$

Então $Q P_{1}(Y \oplus Z) \subset P_{1}(Y \oplus Z)$. 
Além disso, $P_{1}=\left(\begin{array}{cc}A_{1} & 0 \\ 0 & D_{1}\end{array}\right)$, onde $A_{1}$ é uma projeção em $Y$ e $D_{1}$ é uma projeção em $Z$, pois $P_{1}$ é uma projeção em $Y \oplus Z$. Também temos que $(z-P)-(z-Q)=Q-P=$ $S \in \operatorname{In}(Y \oplus Z)$. Então

$$
(z-P)^{-1}-(z-Q)^{-1}=(z-Q)^{-1}((z-Q)-(z-P))(z-P)^{-1} \in \operatorname{In}(Y \oplus Z)
$$

pois In é um ideal de operadores. Portanto,

$$
\begin{aligned}
P-P_{1} & =\frac{1}{2 \pi i} \int_{\Gamma}(z-P)^{-1} d z-\frac{1}{2 \pi i} \int_{\Gamma}(z-Q)^{-1} d z \\
& =\frac{1}{2 \pi i} \int_{\Gamma}\left[(z-P)^{-1}-(z-Q)^{-1}\right] d z \\
& =\frac{1}{2 \pi i} \lim _{k \rightarrow \infty} \sum_{k}\left[(z-P)^{-1}-(z-Q)^{-1}\right]\left(\xi_{k}\right)\left(\lambda_{k}-\lambda_{k-1}\right) .
\end{aligned}
$$

implicando que $P-P_{1} \in \operatorname{In}(Y \oplus Z)$, pois pela Proposição 93 In é um ideal fechado de operadores. Então

$$
P_{1}-P \in \operatorname{In}(Y \oplus Z)
$$

$4^{\mathbf{o}}$ passo.) Definindo $V=I-P+P_{1}$ temos, pela Proposição 116 , que $V$ é um operador de Fredholm e

$$
i(V)=i\left(I+P_{1}-P\right)=i(I)=0
$$

Além disso,

$$
V(M)=V(P(Y \oplus Z))=P_{1} P(Y \oplus Z)=P_{1}(M) \subset R\left(P_{1}\right)
$$

Como $I-\left(P_{1}-P\right) \in \Phi(Y \oplus Z)$ então temos que $\operatorname{codim}\left(R\left(I-P_{1}+P\right)\right)<\infty$ em $Y \oplus Z$ e como

$$
R\left(I-P_{1}+P\right) \subset R\left(I-P_{1}\right)+R(P)=N\left(P_{1}\right)+M .
$$

então, pelo Lema 202, $\operatorname{codim}\left(N\left(P_{1}\right)+M\right)<\infty$ em $Y \oplus Z$. Logo, segue que $\operatorname{codim}(V(M))<$ $\infty$ em $R\left(P_{1}\right)=A_{1}(Y) \oplus D_{1}(Z)$ e deste modo, pela. Proposição 170 , temos que $V_{\left.\right|_{M}}: M \rightarrow$ $R\left(P_{1}\right)$ é um operador de Fredholm de índice zero. Pela Observação 76 existem subespaços $M_{1}$ e $N=N\left(V_{\left.\right|_{M}}\right)$ de $M$ e $V(M)$ e $W$ de $R\left(P_{1}\right) \operatorname{com} \operatorname{dim}(N)=\operatorname{dim}(W)<\infty$ tais que $M=M_{1} \oplus N, V(M) \oplus W=R\left(P_{1}\right)$ e $V_{l_{M_{1}}}$ é um isomorfismo em $Y_{1} \oplus Z_{1}$ para algum $Y_{1} \subset A_{1}(Y)$ e $Z_{1} \subset D_{1}(Z)$. Suponhamos que ambos, $Y_{1}$ e $Z_{1}$ possuem codimensão finita em $Y$ e em $Z$, respectivamente. Então pelo Lema 201 temos que $Y_{1} \oplus Z_{1}$ possui 
Subespaços complementados na soma de espaços de Banach essencialmente 110 incomparáveis

codimensão finita em $Y \oplus Z$. Como $Y_{1} \oplus Z_{1} \subset R\left(P_{1}\right)$ então, pelo Lema 202, temos que $\operatorname{codim}\left(R\left(P_{1}\right)\right)<\infty$ em $Y \oplus Z$. Desde que $P_{1}$ uma projeção então $Y \oplus Z=N\left(P_{1}\right) \oplus R\left(P_{1}\right)$, implicando que $\operatorname{dim}\left(N\left(P_{1}\right)\right)<\infty$. Logo, $P_{1} \in \Phi(Y \oplus Z)$. Por (6.9) e pela Proposição 165 temos que

$$
P=P_{1}-\left(P_{1}-P\right) \in \Phi(Y \oplus Z)
$$

e, deste modo, codim $(R(P))<\infty$, o que contradiz (6.8). Assim, podemos assumir que pelo menos um dos subespaços $Y_{1}$ ou $Z_{1}$ é de codimensão infinita em $Y$ ou $Z$, respectivamente. Vamos assumir que $\operatorname{codim}\left(Y_{1}\right)=\infty$ em $Y$. O outro caso é análogo.

Como $\operatorname{dim}(N)=\operatorname{dim}(W)<\infty$ então consideremos o isomorfismo sobrejetor $\varphi: N \rightarrow$ $W$. Desde que $P$ é uma projeção sobre $M$ então

$$
Y \oplus Z=R(P) \oplus N(P)=M \oplus N(P)=M_{1} \oplus N \oplus N(P) \text {. }
$$

5ㅇ passo.) Consideremos o operador $A: Y \oplus Z=M_{1} \oplus N \oplus N(P) \rightarrow Y \oplus Z$ definido por $A\left(m_{1}+n+w\right)=\varphi(n)$, onde $m_{1} \in M_{1}, n \in N$ e $w \in N(P)$. Temos que $A \in L(Y \oplus Z)$ e tem posto finito. Além disso, se $m_{1}+n+w \in N(A)$, onde $m_{1} \in M_{1}, n \in N$ e $w \in N(P)$, então $0=A\left(m_{1}+n+w\right)=\varphi(n)$, implicando que $n=0$ e portanto $N(A) \subset M_{1} \oplus N(P)$. Obviamente, $M_{1} \oplus N(P) \subset N(A)$. Portanto,

$$
N(A)=M_{1} \oplus N(P)
$$

Temos também que $A(N)=W$ é um subespaço de $Y$ disjunto de $Y_{1}$ pois $Y_{1}$ é de codimensão infinita em $Y$. Segue da Proposição 115 que $V+A$ é um operador de Fredholm de índice 0 . Além disso, $N(V+A) \cap M=\{0\}$ pois se $x \in N(V+A) \cap M$ então $x=m_{1}+n$ e $0=(V+A)(x)=V(x)+A(x)$, implicando que

$$
V(x)=-A(x)
$$

Como $V(x) \in V(M),-A(x) \in W$ e $\dot{W} \cap V(M)=\{0\}$, então segue de (6.13) que $V(x)=A(x)=0$. Desde que $0=A(x)=A\left(m_{1}+n\right)=\varphi(n)$, temos que $n=0$ e então $x=m_{1} \in M_{1}$. Por outro lado, como $V(x)=0$ então $x \in N$. Logo, $x=0$. Portanto,

$$
N(V+A) \cap M=\{0\}
$$

6모sso.) Como $i(V+A)=0$ então $\alpha(V+A)=\beta(V+A)<\infty$, implicando que $\operatorname{dim}(N(V+A))=\operatorname{dim}(X)<\infty$, onde $Y \oplus Z=X \oplus R(V+A)$. Portanto, existe 
um isomorfismo $u: N(V+A) \rightarrow X$ e uma projeção $P^{\prime}: Y \oplus Z \rightarrow N(V+A)$. Seja. $k=u \circ P^{\prime}: Y \oplus Z \rightarrow X$. Temos que se $x \in M$ e $x \neq 0$ então por (6.14) temos que $x \notin N(V+A)$, implicando que $k(x)=u\left(P^{\prime}(x)\right)=u(0)=0$. Portanto $x \in N(k)$ e então $M \subset N(k)$.

7으 passo.) O operador $U=V+A+k$ é bijetor. De fato, temos que se $x \in N(V+A+k)$ então $0=(V+A+k)(x)=(V+A)(x)+k(x)$, implicando que $(V+A)(x)=-k(x)$. Como $(V+A)(x) \in R(V+A)$ e $k(x) \in R(k), R(k) \subset X$ e $X \cap R(V+A)=\{0\}$ então segue que $(V+A)(x)=k(x)=0$. Logo $(V+A)(x)=0$, implicando que $x \in N(V+A)$.

Sendo $u$ um isomorfismo temos que $0=k(x)=u\left(P^{\prime}(x)\right)$ e então $P^{\prime}(x)=0$, implicando que $x \in C$, onde $Y \oplus Z=C \oplus N(V+A)$. Logo $x=0$. Então $N(V+A+k)=\{0\}$, implicando que $\alpha(V+A+k)=0$. Segue da. Proposição 115 que $i(V+A+k)=i(V+A)=0$ e daí obtemos que $\beta(V+A+k)=0$ e, portanto, $U$ é bijetor.

Denotando $Y_{o}=Y_{1} \oplus R(A)$ e $Z_{o}=Z_{1}$ temos que $U$ é um isomorfismo de $M$ sobre $Y_{o} \oplus Z_{o}$ e a prova está completa.

Finalmente destacamos que permanece em aberto o seguinte problema listado em [3]:

Problema. Sejam $X$ e $Y$ espaços de Banach tais que $L(X, Y)=\operatorname{Imp}(X, Y)$. É verdade que todo subespaço complementado de $X \oplus Y$ é isomorfo à soma de um subespaço complementado de $X$ com um subespaço complementado de $Y$ ? 


\section{Referências Bibliográficas}

[1] Aiena, P. e González, M., Essentially Incomparable Banach Spaces and Fredholm Theory, Proc. R. Ir. Acad. 93A (1993), 49-59.

[2] Aiena, P. e González, M., Examples of Improjective Operators, Math. Z. 233 (2000), 471-479.

[3] Aiena, P. e González, M., On Inessential and Improjective Operators, Studia Math. 131 (1998), 271-287.

[4] Aiena, P., On Riesz and Inessential Operators, Math. Z. 201 (1989), 521-528.

[5] Caradus, S. R., Pfaffenberger, W. E. e Yood, B., Calkin Algebras and Algebras of Operators on Banach Spaces. New York, 1974.

[6] Dunford, N. e Schwartz, J. T., Linear Operators - Part I: General Theory. Wiley Classics Library, 1988.

[7] Edelstein, I. S. e Wojtaszczyck, P., On Projection and Unconditional Bases in Direct Sums of Banach Spaces, Studia Math. T. LVI (1976), 263-276.

[8] Goldberg, S., Unbounded Linear Operators. Theory and Applications. McGraw-Hill, inc., 1966.

[9] Gohberg, I. e Goldberg, S., Basic Operator Theory. Birkhaüser, Boston, 1981.

[10] González, M., On Essentially Incomparable Banach Spaces. Math. Z. 215 (1994), 621-629. 
[11] Gowers, W. T. e Maurey, B., The uncounditional Basic Sequence Problem. J. Amer. Math. Soc. 6 (1993), 851-874.

[12] Gowers, W. T. e Maurey, B., Banach Spaces with small spaces of Operators. Ma.th.Ann. 307 (1997), 543-568.

[13] Grothendiek, A., Sur les applications linéaires faiblement compactes d'espaces du type C(K). Canad. J. Math. 5 (1953), 129-173.

[14] Heuser, H., Functional Analysis. Wiley \& sons, 1982.

[15] Kleinecke, D., Almost-finite, compact, and inessential operators. Proc. Amer. Math. Soc. 14 (1963), 863-868.

[16] Lebow, A. e Schechter, Semigroups of Operators and Measures of noncompactness. J. Funct. Anal. 7 (1971), 1-26.

[17] Lima, E. L., Curso de Análise - Volume 1. Instituto de Matemática Pura e Aplicada. Rio de Janeiro, 1995.

[18] Lima, E. L., Curso de Análise - Volume 2. Instituto de Matemática Pura e Aplicada. Rio de Janeiro, 1995.

[19] Lima, E. L., Espaços Métricos. Instituto de Matemática Pura e Aplicada. Rio de Janeiro, 1993.

[20] Lindenstrauss, J. e Tzafriri, L., Classics Banach Spaces I. Sequence Spaces. New York, Springer, 1977.

[21] Lindenstrauss, J., On complemented subspaces of m. Israel J. Math. 5 (1967), 153-156.

[22] Pelczynski, A., Projection in certain Banach spaces. Studia Math. 19 (1960), 209-228.

[23] Pietsch, A., Inessential Operators in Banach Spaces. Integral Equations and Operators Theory 1 (1978), 589-591.

[24] Pietsch, A., Operators Ideals. North-Holland; Amsterdan; New York, Oxford, 1980.

[25] Pietsch, A., Theorie der Operatorenideale. Wissenschaft. Beiträge der Uni. Jena. 
[26] Prezeworska-Rolewicz, D. e Rolewicz, S., Equations in Linear Spaces. Warsaw, 1968.

[27] Semadeni, Z., Banach spaces of continous functions. PWN, Warszawa, 1971.

[28] Schechter, M., Riesz Operator and Fredholm Pertubations. Bull. Am. Math. Soc. 74 (1968), 1139-1144.

[29] Tarafdar, E., Improjective Operators and Ideals in a Category of Banach Spaces. J. Austral. Math. Soc. 14 (1972), 274-292.

[30] Tarafdar, E., On Further Properties of Improjective Operators. J. Austral. Math. Soc. 14 (1972), 352-363.

[31] Taylor, A. E., Introduction to Functional Analysis. Wiley \& Sons, New York, 1958.

[32] Weis, L., Pertubation classes of semi-Fredholm operators. Math. Z. 178 (1981), 429442.

[33] Wojtaszczyk, P. e Edelstein, I. S., On projections and uncounditional bases in direct sums of Banach Spaces. Studia Math. 56 (1976), 263-276.

[34] Wojtaszczyk, P. On projections and unconditional bases in direct sum of Banach Spaces II. Studia Math. 62 (1978), 193-201. 